\title{
ABORIGINAL WOMEN'S EXPERIENCES OF ACCESSING HEALTHCARE WHEN STATE APPREHENSION OF CHILDREN IS BEING THREATENED
}

\author{
by \\ JACQUELINE DENISON
}

B.S.N., The University of British Columbia, 2004

\begin{abstract}
A THESIS SUBMITTED IN PARTIAL FULFILLMENT OF THE REQUIREMENTS FOR THE DEGREE OF

MASTER OF SCIENCE IN NURSING

in

THE FACULTY OF GRADUATE STUDIES
\end{abstract}

(Nursing)

THE UNIVERSITY OF BRITISH COLUMBIA

(Vancouver)

April 2012

(C) Jacqueline Denison, 2012 


\begin{abstract}
Background: Canadian Aboriginal children continue to be apprehended at a higher rate than non-Aboriginal children. Aboriginal mothers, who face significant social, political and economic disadvantage, are often under considerable scrutiny in relation to their parenting. Little is known about how the threat of child apprehension impacts the experiences of Aboriginal women accessing healthcare services. Therefore a study was undertaken to examine women's perspectives on accessing health care when child apprehension is threatened. Methods: The study was guided by post-colonial feminist perspectives and followed the principles of exploratory, qualitative research design. Data collection and data analysis were carried out in two phases. Phase One involved a secondary analysis, using narrative interview data collected from a larger study $(n=7)$. Phase One findings were used primarily to modify the interview guides developed for Phase Two. In Phase Two primary, face-to-face interviews were conducted with (i) Aboriginal women (N=9) and (ii) health care providers $(n=8)$. Data was analyzed following the principles of thematic analysis and interpretive description. Findings: The findings indicated that women involved in the child protection system often experience complex socio-political and economic life challenges. The threat or fear of child apprehension did not impact the women's decisions to seek medical services for their children; however the threat and fear impacted the women's experiences with mainstream healthcare in a number of ways. Racism, prejudice, and discrimination within mainstream healthcare agencies along with the fear of child apprehension influenced the women's decisions to access healthcare for themselves and impacted the women's interactions with mainstream health care providers. In particular, women avoided engaging with health care providers when their children were hospitalized
\end{abstract}


in part to protect themselves from judgment and discrimination. Discussion: Ongoing racism, judgment and discrimination toward Aboriginal mothers in mainstream healthcare agencies must be addressed. Health care providers working with Aboriginal people require education around culturally safe approaches to care and the history of colonialism and its effects on the health and well-being of Aboriginal people. Structural inequities such as poverty and discrimination must also be addressed through policy initiatives that attend to the social determinants of health. 


\section{PREFACE}

This study is closely linked to a larger study funded by the Canadian Institute of Health Research entitled, Improving access to primary healthcare: Lessons learned from two urban Aboriginal Health centres. Principal Investigator: Annette Browne. Co-Investigators: Olive Godwin, Josee Lavoie, Doreen Littlejohn, Heather Louise Mac Donald, John O'Neil. Patricia Rodney, Victoria Smye, Lisa Tabobondung, David TU, Colleen Varcoe and Sabrina Wong. The data collected in Phase One was previously collected in-depth, narrative interview data from the larger study. This study underwent external review by CIHR and UBC REB. The UBC BREB number is $\mathrm{H} 07-02010$.

This study was approved by the University of British Columbia's Behavioral Research Ethics Board (UBC BREB). The UBC BREB number for this study is: H10-02413.

This study also received approval by Vancouver Coastal Health Authority - Vancouver Community, Certificate \# V11-02413. 


\section{TABLE OF CONTENTS}

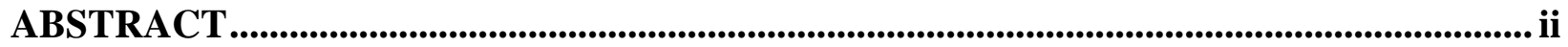

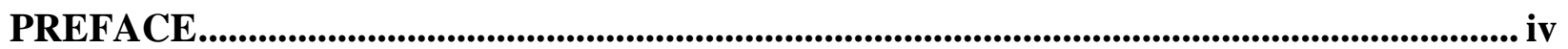

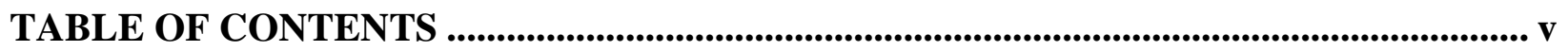

ACKNOWLEDGMENTS ....................................................................................................... vii

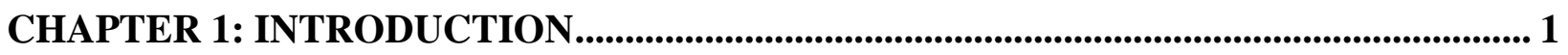

Background to the Problem ................................................................................................. 1

Purpose and Research Questions ....................................................................................... 4

CHAPTER 2: REVIEW OF THE LITERATURE......................................................... 6

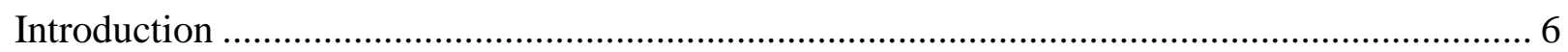

Theoretical Perspectives Informing the Research: Post-Colonial Theory and Post-Colonial

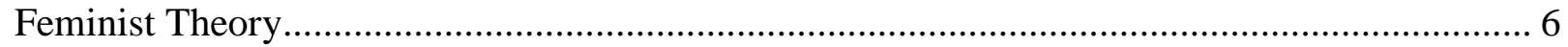

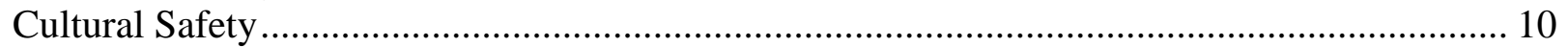

A History of Government-Induced Disruption in Aboriginal Mothering ................................. 12

Child Abuse and Neglect in Context ................................................................................ 16

The Child Protection System in British Columbia .................................................................. 18

Current Issues Related to Aboriginal Children in State Care.................................................... 19

Health, Healthcare, and Aboriginal Women and Children........................................................ 23

CHAPTER 3: METHODS ….................................................................................................... 25

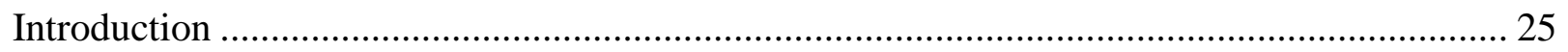

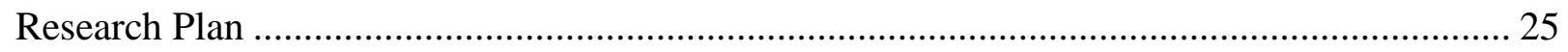

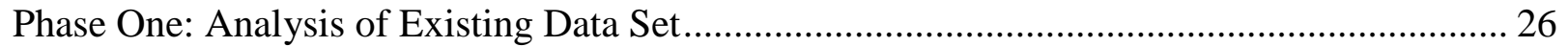

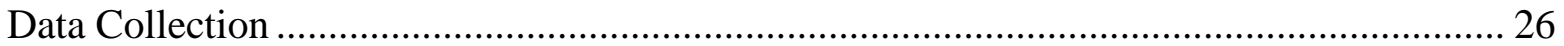

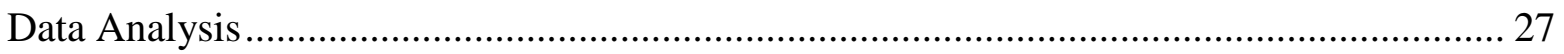

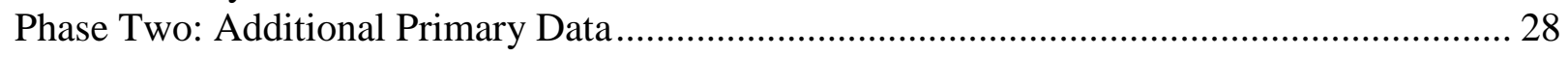

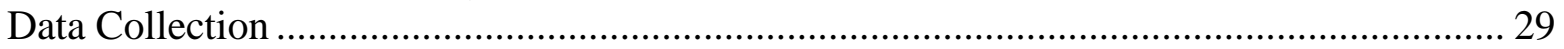

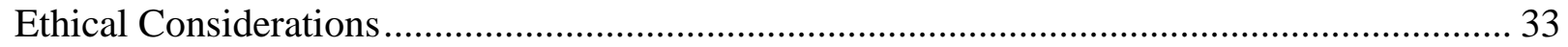

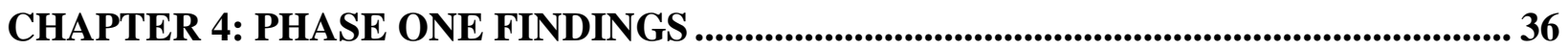

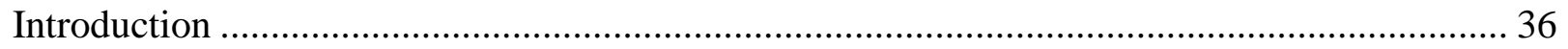

Socio-Economic Factors Influencing Women's Lives .............................................................. 36

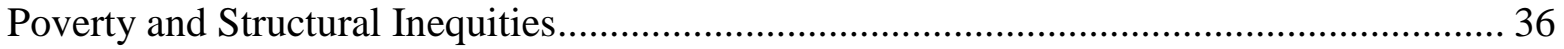

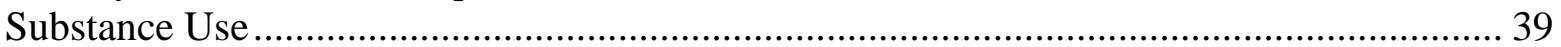

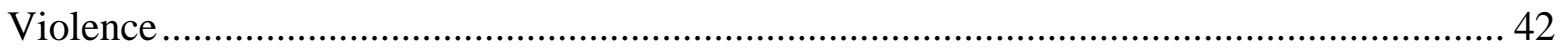

Fathers and Partners ........................................................................................................... 45

Accessing Healthcare Services When Child Apprehension is a Threat .................................... 47

Conclusion: The Basis for Follow-up Questions for Phase Two ............................................... 49

CHAPTER 5: PHASE TWO FINDINGS ............................................................................. 50

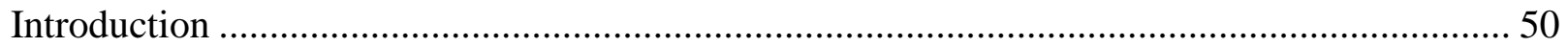

Socio-Political and Historical Context of Women's Lives ....................................................... 50 
Ongoing Disruption of Aboriginal Families and Communities ....................................... 51

Structural Inequities and the Context of Poverty......................................................... 54

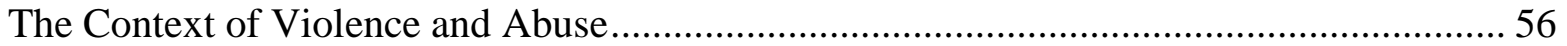

The Bureaucratic Structures Governing Child Protection Agencies ..................................... 59

A Lack of Consistency.......................................................................................... 59

Power of the Child Welfare System ............................................................................ 62

Limited Supportive Programs and Services within the Child Protection System ............... 64

Aboriginal Women's Experiences with The Child Protection System and Process ................. 67

Common terms used within the Child Protection System ................................................ 68

Traumatic Experiences with the Child Protection System ........................................... 72

Early Referral to MCFD .......................................................................................... 78

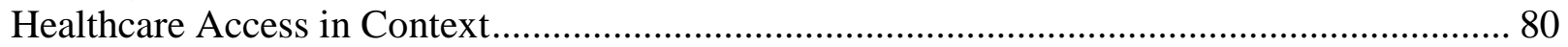

Ongoing Racism and Discrimination in the Healthcare Setting ..................................... 81

Setting up for Success While Navigating a Complex System............................................. 89

Empowering Women and Working Collaboratively with Women and Families ................ 89

Educating Healthcare Staff towards Culturally Safe Care ........................................... 96

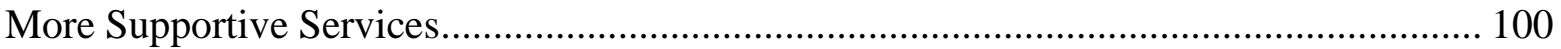

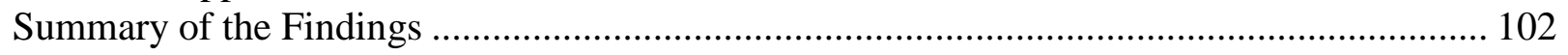

CHPATER 6: DISCUSSION AND RECOMMENDATIONS......................................... 103

Introduction ................................................................................................... 103

How does the Fear of Child Apprehension Impact Experiences with Mainstream Healthcare?

Experiences of Interpersonal Discrimination in Mainstream Healthcare.............................. 105

Structural Poverty and Structural Discrimination ...................................................... 107

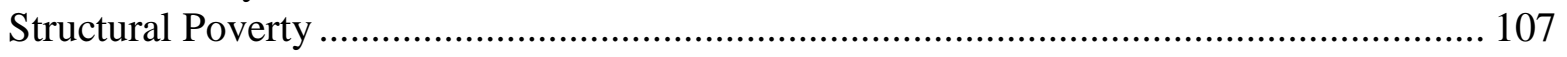

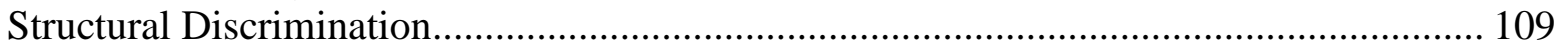

Legislative Authority within the Child, Family and Community Services Act ................ 110

Lack of Supportive Services ................................................................................ 113

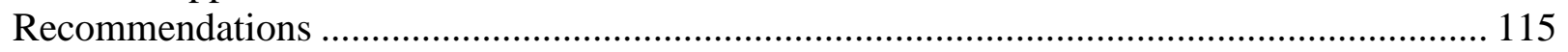

Inter-Sectoral Efforts on Reducing Poverty ........................................................... 115

Improving the Process of Child Apprehensions in the Hospital Environment................... 116

The Provision of Culturally Safe Training to Health Care Providers ................................. 118

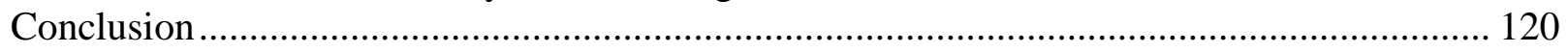

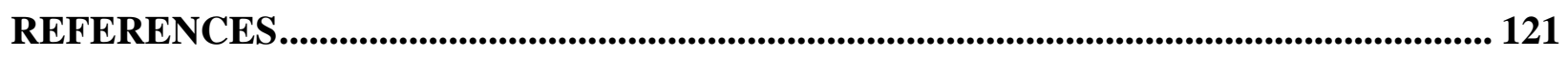

APPENDIX A: INTERVIEW FORMS FOR HEALTH CARE PROVIDERS ................... 132

APPENDIX B: INTERVIEW FORMS FOR WOMEN........................................................... 136

APPENDIX C: CONSENT FORM FOR WOMEN ................................................................... 140

APPENDIX D: CONSENT FORM FOR HEALTH CARE PROVIDERS........................... 143 


\section{ACKNOWLEDGMENTS}

I would like to thank a number of people for their contributions to this study. This thesis is the end result of many people's rich perspectives and I thank everyone that contributed. I first want to thank my committee members; thank you to Dr. Colleen Varcoe and Dr. Annette Browne for sharing your expertise in qualitative research and for your endless support. Their personal work in critical research in health and health inequities largely influenced this study. Thank you to Laraine Michalson for your valuable contributions, and for sharing your expertise, work experience, and knowledge on this topic.

This study is very closely linked to a larger study entitled "Improving Access to Primary Health Care: Lessons from Two Urban Aboriginal Health Centres.” Thank you to Dr. Annette Browne and Dr. Colleen Varcoe for the opportunity to train in a research fellowship on this study. Thank you to Dr. Koushambhi Khan and Alycia Fridkin for all of the intellectual guidance and support that you have given me throughout this fellowship.

Thank you to the Sheena Davidson Nursing Scholarship for helping to fund this study. I am very grateful for the financial support that made this study possible.

Thank you to my parents and my sisters for encouraging me to take on this degree. Liz and Stephanie, your own academic accomplishments have been a great source of motivation for me throughout this process.

Finally, I would especially like to thank all of the women and health care providers that participated in this study. To the women, I am so grateful that you were willing to share your personal stories with me and I am inspired by your strength and resilience. To the health care providers, you are the true experts in this field and I thank you for sharing your experiences and perspectives. 


\section{CHAPTER 1: INTRODUCTION}

\section{Background to the Problem}

The day began like any other morning at work, with handover from the night nurse. After receiving report on the physical status of the infant I would be caring for over the subsequent 12 hours, I asked if the infant's mother had been in to see her baby yet. The night nurse relayed that the mother was still a patient at a different hospital; her infant needed to be transferred to a more intensive facility and thus was sent to this tertiary Neonatal Intensive Care Unit (NICU).

My impression of the infant was that she was sick but not critically ill; she needed antibiotics and some respiratory support. I read her chart but little information was available at the time. I learned that this was not the mother's first child and that the mother used cocaine, cigarettes, and alcohol during her pregnancy. The father of the infant was said to be violent and for that reason her other children were not in either parents' care. The admission note relayed

that the mother came into the hospital complaining of severe abdominal pain after possibly being kicked by her partner in the stomach, and was found to be in pre-term labor.

Late in the morning I received a phone call from the social worker assigned to the infant's mother. She "reassured" me that the paper work was signed and being processed and that everything was underway for the ministry to apprehend the child. I asked why it was so urgently decided that this child was not able to be in the care of its mother. The social worker informed me that the reason for the apprehension was because the mother lives in an environment that is unsafe and that she has been told several times that in order for her to gain guardianship of her children she must leave her partner and the place she is living in, but she refuses. 
It struck me that this woman was told to leave an environment to maintain the best interest of her children. I wondered what alternatives this mother had in regards to her living arrangements and why it was acceptable for her to be in a violent and unsafe environment but not her children. Additionally, I was confused as to how a child could be apprehended hours after a mother had given birth. Later that afternoon, an Aboriginal woman came to visit the infant. She referred to herself as an auntie, clarifying that this is how they define their relationship but that she was not a blood relative. She said she was a member of the mother's band and had just spoken to the infant's mom. I gave the woman a quick update on the infant's status; she appeared relieved and then said, " [The mom] is worried that she signed something she shouldn't have this morning... someone came into the room when she was out of it on morphine and asked her to sign something, and she is worried about what she signed. "It was at that moment that I realized the mother signed away her child, and was not even aware of what she had done.

At the end of the day I left wondering what would happen to this family. I thought about this mother, and felt great sadness for her decision to enter a hospital for help and have the final result be the loss of a child. My questions continued: how is it determined that a mother is not able to care for her child hours after the birth and who does it serve to decide this so quickly? How does taking an infant from his or her biological mother impact the health and well-being of the mother and the infant? And, how does being Aboriginal impact receiving mainstream maternal-child healthcare services?

This experience occurred while working as a neonatal nurse at a large tertiary hospital and mid-way through my graduate studies, which have focused on critical cultural inquiry and the health inequities experienced by Canada's marginalized populations, particularly the 
Aboriginal $^{1}$ people in Canada. The event had a great impact on this study and was the beginning of my inquiry into the experiences of Aboriginal women accessing healthcare services when apprehension of their children is threatened by the child protection system ${ }^{2}$.

There continues to be a distressingly high rate of Canadian Aboriginal children in government care (Browne, MacDonald \& Elliot, 2009; Palmer \& Cooke, 1996; Farris-Manning \& Foster, 2003). Statistics indicate that from 2000-2002 an estimated 76, 000 children were in government care in Canada (Trocmé, Knoke, \& Blackstock, 2004). This number was based on the 2000-2002 annual provincial and territorial reports from child and family services; however, it is only an estimate, as reporting systems are both inadequate and incomplete (Canadian Counsel of Provincial Child and Youth Advocates, 2010; Trocmé, et al.). Aboriginal children and youth represent approximately $30-40 \%$ of children in government protective services across Canada, with large variances among the provinces and territories (Trocmé, et al.; Farris-Manning \& Foster; Fournier \& Crey, 1997). In British Columbia, Aboriginal children compromise approximately $8 \%$ of the total number of children while representing $54 \%$ of the total number of children in ministry care (Ministry of Child and Family Development, 2009/10).

There have been significant improvements in Aboriginal peoples' health over the last few decades; however Aboriginal women and children's health continues to fall significantly below that of non-Aboriginal Canadian women and children (Browne, MacDonald, \& Elliot, 2009; Canadian Institute of Health Research, 2008b; Adelson, 2005; Bourassa, McKay-McNabb \& Hampton, 2004; Canadian Institute of Health Information, 2004). Aboriginal children continue

\footnotetext{
${ }^{1}$ Throughout this proposal " $[\mathrm{t}]$ he term Aboriginal peoples refers to organic political and cultural entities that stem historically from the original peoples of North America, rather than collections of individuals united by so-called 'racial' characteristics." (Royal Commission Report on Aboriginal People, 1996, volume 1). The term includes the First Nations (also referred to as "Status Indian" and "non-Status Indian"), Métis, and Inuit people of Canada.

${ }^{2}$ The terms child protection system, child protection services, the child welfare system and the Ministry of Child and Family Development (MCFD) will be used interchangeable to refer to any services that have the legislative authority to carry out British Columbia's Child, Family, and Community Services Act.
} 
to be apprehended by child protection services at a higher rate than non-Aboriginal children in Canada yet the reason for the disproportionately higher rate of Aboriginal children in government care is still not well understood. For many Aboriginal women, social, political and economic factors such as poverty, inadequate housing, unemployment, substance use, and violence, largely stemming from a history of colonialism and ongoing neocolonial polices, have created a depiction of these women as neglectful mothers. The challenge of living under the fear of having one's child apprehended undoubtedly affects the decisions a mother makes in her daily living.

A greater understanding of how the fear of child protection investigations (i.e., the fear that children will be removed) impacts how or when Aboriginal women living with social stressors such as poverty, substance use, or violence, access healthcare services is necessary to better facilitate the safe access to healthcare services for these women and their children. There is a wealth of literature that highlights the social, political and economic inequities that Aboriginal mothers experience and there is a large amount of literature exposing ongoing occurrences of child abuse in Aboriginal communities; however, there are minimal support programs available to support the health and well-being of Aboriginal women and children simultaneously living with multiple social stressors (O’Donnell et al., 2010).

\section{Purpose and Research Questions}

The purpose of this research was to explore how the threat of child apprehension affects Aboriginal women and children's experiences accessing healthcare services. Specifically the research questions were: 1) What are Aboriginal women's experiences accessing healthcare services when child apprehension is a threat? 2) What are the perspectives of health care providers who have extensive experience working with Aboriginal women who (i) have 
experienced apprehension of their children or (ii) have had apprehension of their children threatened by the state? 3) How can the quality and safety of healthcare delivery to Aboriginal women and children be improved when child apprehension is a threat? 


\section{CHAPTER 2: REVIEW OF THE LITERATURE}

\section{Introduction}

This chapter begins with a description of post-colonial and post-colonial feminist perspectives. Post-colonial and post-colonial feminist theories are helpful in understanding how issues such as poverty, violence, gender, and past and present day discriminatory government policies intersect and shape certain groups' health and well-being. In this study, post-colonial and post-colonial feminist theory are taken to assist in understanding how some Aboriginal women's health and well-being have been negatively impacted by stressors such as poverty, violence, and oppressive government policies. Following this is a review of the literature, organized into five subsections: A history of government disruption in Aboriginal mothering; child abuse and neglect in context; the child protection system in British Columbia; current issues with Aboriginal children in state care; and health, healthcare, and Aboriginal women and children.

\section{Theoretical Perspectives Informing the Research: Post-Colonial Theory and Post-Colonial Feminist Theory}

Post-colonial theory is a critical form of inquiry; it is known as a multidisciplinary perspective, influenced by other critical theories such as, postmodernism, poststructuralism, feminism and neomarxism (Reimer-Kirkham, \& Anderson, 2002; Anderson, 2004; ReimerKirkham, Baumbusch, Schultz, \& Anderson, 2007, Anderson, 2002). Post-colonial perspectives originated in the 1960s-1970s by anticolonial scholars such as Edward Said and Frantz Fanon; however many other scholars have contributed rich perspectives (Reimer-Kirkham \& Anderson, 2002). Post-colonial feminist theory is valuable in understanding Aboriginal women's health as 
it exposes how racialization, historical subjugation, culture and class are social conditions that interact with gender to shape life opportunities and health (Browne, Smye \& Varcoe, 2007).

I begin here with the relevant concepts from post-colonial theory, which include: 'race,' racialization, 'othering' and culture (Browne et al., 2005). Historically the term 'race' referred to a person's biological origin and physical appearance; however a biological component to race does not exist. I place the term 'race' in quotations throughout this chapter to indicate the meaning in which I use the term, which is "...[a] social construction manipulated to define, structure, and organize relations between dominant and subordinate groups." (Reimer-Kirkham \& Anderson, 2002, p, 4). Racialization is defined as "a political and ideological process by which particular populations are identified by direct or indirect reference to their real or imagined phenotypical characteristics" (Anderson, 2002, p. 13). The term 'othering' refers to the process of ascribing assumed cultural characteristics, "differences," or identities to people from particular groups (Browne et al., 2005). The assumed 'cultural' characteristics and identities projected onto certain people are created from stereotypical identities rather than real identities (Browne et al., 2005). The process of 'othering' in reference to indigenous people is committed through racialization (Browne \& Varcoe, 2006). Culture is a particularly difficult concept to define; the meaning of culture has been ".... historically and politically mediated, and continues to be influenced by our ever-changing political and economic climate" (Browne \& Varcoe, p. 157). Through the lens of post-colonial theories, culture is seen as a fluid concept. Anderson (2002) cites Bhabha's work. In the late $20^{\text {th }}$ century, Bhabha coined the idea of a space for cultural hybridity. From this viewpoint, culture is seen as vague and constructed through a negotiated process. Culture, as a socially constructed concept, is not neutral; it is the result of unequal power relations between the colonizer and the colonized (Anderson). Thus the meaning 
or construction of culture is not static, but rather ever changing through processes of colonialization, diaspora and displacement. 'Race', culture and the process of racialization (i.e., 'othering') are socially produced meanings that are conceived through historical, economic, and political processes of colonialization and imperialism (Anderson).

One of the main objectives of post-colonial theory is to attend to the relations and repercussions of past colonial policies as well as ongoing neocolonialism (Reimer-Kirkham et al., 2007; Reimer-Kirkham \& Anderson, 2002). Post-colonial scholars have created a discourse that provides a voice to the colonized, by promoting alternative perspectives on knowledge production. Moreover, post-colonial theory challenges who has the authority to produce knowledge, (Anderson, 2002) “... [as] it is from those who have suffered the sentence of history - subjugation, domination, diaspora, displacement - that we learn our most enduring lessons for living and thinking (Bhabha, 1994, p. 172). Browne, Smye, \& Varcoe (2007) relay that a unique quality of post-colonial theory is that it "...disrupt[s] the enduring history of 'race thinking' and structural inequities that have been brought about by histories of colonialization and ongoing neocolonial practices" (p. 3).

Post-colonial theory provides an analytic tool that allows one to examine how the nonwestern 'other' has been socially constructed by dominant western society (Anderson, 2002). It does not refer to one specific colonized group, nor to a specific geographical location; postcolonial theory is relevant in all nations worldwide (Anderson). Additionally, post-colonial theory does not refer to one particular moment in time but instead refers to the aftermath of colonialization and the neocolonial policies of today (Anderson; Browne et al., 2005; Browne, Smye, \& Varcoe, 2007). Thus, the 'post' in post-colonialism is not to imply that colonialism is an issue of the past; new power imbalances exist today through neocolonial policies that add 
complexities to the history of those who have been categorized as 'other' by the dominant western society (Browne, Smye, \& Varcoe, 2007). The process of 'othering' particularly has affected non-European women in Canada, who through socially constructed concepts of 'race' and culture, often have been categorized as the inferior 'other' (Browne, Smye, \& Varcoe, 2007)

Originally post-colonial theory did not include a gender analysis; rather, scholars have added a gender analysis to this perspective. Post-colonial feminist theory joins the concepts of feminist theory to the perspectives of post-colonial inquiry in a way that potentiates the 'explanatory powers' of both perspectives (Browne, Smye, \& Varcoe, 2007). Integrating feminist and post-colonial theories allows a gender analysis of the social and historical experiences of men and women. Subsequently, post-colonial feminist theory “...directs attention to multiple intersecting oppressions, inclusive of gender, class, and race oppression, to reveal the multiple dimensions of oppression within societies, and the unequal effects of racism on certain groups of people (e.g. women and children).” (Reimer-Kirkham et al., 2007, p. 29). Including a gender analysis highlights the different experiences of men and women, and emphasizes the unique experiences of the individual (Anderson, 2002).

Post-colonial feminist scholarship provides a strong lens for knowledge construction from the perspective of the marginalized women whose voices have previously been silenced (Anderson, 2002). The concept 'intersectionality' is particularly relevant in linking post-colonial and feminist theory (Browne, Smye, \& Varcoe, 2007). Intersectionality is understood as the ability of social phenomena such as, 'race,' gender and class to mutually create one another's meanings (Browne, Smye, \& Varcoe, 2007). Anderson (2002) examined post-colonial and black feminist theory together to bring attention to the challenging issues at the intersections of gender, 'race', class relations, and culture, as they relate to women's health and well-being. Anderson 
highlighted the concepts that address discourses and actions permitting the categorizing of people into racial categories. 'Racial' categorizing situates one group as inferior to the other, creating hierarchies among groups. Post-colonial theories provide a way of thinking that examines the concepts of 'race', racialization, 'other' and culture to shed light on how socially constructed meanings of 'race' and culture have allowed the categorizing of people into the nonwestern 'other' contrasted against dominant western people (Browne, Smye, \& Varcoe, 2007).

It is undeniable that Aboriginal peoples' health has been impacted greatly by social relations ingrained in Canada's colonial history and ongoing neocolonial policies (Browne et al, 2005). Post-colonial feminist theory is integral in critically examining why more Aboriginal women experience poorer health and worse access to healthcare services than non-Aboriginal women by drawing explicit attention to a number of the underlying factors that have shaped many Aboriginal women's experiences with westernized healthcare services. Post-colonial feminist perspectives questions why certain people in Canada experience higher rates of illness and disease. It challenges the idea that some people experience higher rates of illness solely because of some sort of biological predisposition; rather, post-colonial feminism brings attention to how social, political and economic factors continue to disadvantage certain groups of people in Canada. Next I discuss cultural safety; a relatively new discourse in nursing literature, that has been developed to address some of the inequities in health and access to health experienced by certain ethnocultural groups.

\section{Cultural Safety}

Cultural safety is a concept that emerged out of New Zealand in the 1990s from Maori nurse leaders in partnership with the Maori people. More recently the concept of cultural safety has been brought into Canadian nursing literature (Browne, Varcoe et al., 2009; Browne \& 
Varcoe, 2006; Browne et al., 2005). Cultural safety draws on the concepts of post-colonial theory to bring attention to existing power imbalances between certain groups of people, and helps one to recognize how existing power imbalances shape people's health and access to healthcare (Browne et al., 2005; Browne, Varcoe et al. 2009). Browne and Varcoe (2006) discuss how culture has been most commonly defined in Western society's health and healthcare discourses. The most widely used definition of culture in Western society is a static definition that focuses on the values, beliefs, knowledge and customs of groups of people, with no regard to racism, discrimination and colonialism (p. 158). This narrow definition of culture has been taken up in 'culturalist' perspectives, but it can be hazardous as it runs the risk of grouping or categorizing people based on their 'cultural traits' or 'cultural differences' (i.e., food, customs, clothing, etc.). Further, the caution of a 'culturalist' definition of culture is that it can lead to 'othering' or racialization (concepts discussed previously) because it does not take into account how some groups of people (e.g., Aboriginal people) have been categorized as 'different' or 'other' and often inferior to dominant Western society.

The definition of culture applied in the discourse of cultural safety is congruent with the definition taken up in the critical cultural perspectives (as described earlier in this chapter), Browne and Varcoe (2006) defined culture as, “... a relational aspect of ourselves that shifts over time depending on our history, our past experiences, our social, professional and gendered location, and our perceptions of how we are viewed by others in society." (p. 162). Thus culture is viewed as a socially constructed, non-neutral, fluid concept that constantly changes over time.

A culturally safe approach to providing healthcare strives to prevent health care providers delivering care that could create a 'cultural risk.' (Browne, Varcoe et al., 2009, p. 169). A culturally unsafe situation occurs when a member from one ethnocultural group feels he or she 
has been demeaned or disempowered by people from another culture (Browne, Varcoe et al., 2009, p. 169). Culturally safe approaches attempt to diminish such demeaning interactions and aim to create a more 'safe' environment within the health and political arenas (Smye \& Browne, 2002). The framework of culturally safe care is particularly helpful in Canada for understanding why certain health inequities exist for some Aboriginal people. Health care providers need to be educated about the effects of colonization on Aboriginal people to better understand how oppressive government policies have negatively impacted many Aboriginal people's access and experiences in mainstream healthcare. This teaching must address the Indian Act (1876) and the creation of the residential school system as well as the intergenerational trauma experienced by many Aboriginal people (Browne, Varcoe et al., 2009).

\section{A History of Government-Induced Disruption in Aboriginal Mothering}

Despite significant improvements, Aboriginal people continue to experience poorer health than non-Aboriginal Canadians (Canadian Institute of Health Research, 2008b; Adelson, 2005; Bourassa et al., 2004; Canadian Institute of Health Information, 2004). This is not based on any inherent characteristic of Aboriginal people but rather is one of the outcomes of experiencing higher rates of poverty, political disadvantage, lower social status, inadequate housing, a history of trauma related to colonial policies and state intervention, and higher rates of violence. It is indisputable that Aboriginal women face increased vulnerability to poorer health; this is largely due to policies created in the original Indian Act (1876) as they intersect with ongoing gender and racialized oppression that characterizes Canadian society today. Understanding women's health through post-colonial feminist theory demands one to consider how politics and policy shape people's lives. 
Prior to the creation of the Indian Act (1876) no single definition for "Indians" existed; First Nations people were free to define themselves (Armitage, 1995; Turpel, 1993). In 1850 the Canadian civil government created the first legal definition of "Indian," outlining who was and who was not recognized as an "Indian." “...[T]he civil government, an agency beyond the control of Indians, a body in which Indians were not even eligible to have representation, arrogated to itself the authority to define who was and who was not an Indian" (as cited by Armitage, p.84). The legal definition for "Indian" under the original Indian Act in 1876 named anyone who was a male person of Indian blood recognized to belong to a particular band; any child of any such person; or any woman who was or is married to any such person a legal (“status") 'Indian' (Armitage). Although amendments have been made to the original Indian Act in an attempt to remove some of the more overt racist and sexist policies, the implications of past policies continue to exist and "status" continues to be granted in favor of males that are First Nations (Armitage; Bourassa et al., 2004). Subsequently, Aboriginal women and their children particularly have suffered the negative outcomes of colonial policies.

Traditional Aboriginal values included Aboriginal women as being highly regarded in their homes and communities. Turpel (1993) described how in traditional teachings by Cree Elders, Cree women were viewed as being at the centre of the circle of life. "It is women who give birth both in the physical and in the spiritual sense to the social, political and cultural life of the community." (Turpel, p.180). Historically, in First Nations communities' women felt complete, at the centre of their environment and were respected in the roles they assumed (Turpel). However, as previously stated, interactions with European settlers and discriminatory government policies have impacted the lives and roles of Aboriginal women. "[Aboriginal] women have their lives disrupted by state intervention to a greater degree than any other women 
of Canada and more than their male Aboriginal peers" (Fiske, 1995 p.3). In the late twentieth century a number of activists and Aboriginal women lobbied for changes to the Indian Act (1876) in an attempt to promote sexual equality (namely Bill C-31). Amendments were made to the Indian Act to improve the equality in gaining "Indian status" for First Nations men, women and their children (Turpel). However, these amendments have created even more complex issues of inequities for Aboriginal women, in part because women who attempt to regain their status are returning back to communities that have limited resources (Fiske; Bourassa, et al., 2004).

One of the first and foremost disruptions into the parenting practices of Aboriginal families was the state's creation of the residential school system. The first Canadian boarding school for Aboriginal children was opened in 1620 by the Recollects in New France (Greenwood \& De Leeuw, 2007). Children as young as three years old were taken from their homes and placed into residential schools (Ing, 2006). The residential school system was instrumental in building a barrier between Aboriginal children and their families (Ing; Greenwood \& De Leeuw, 2006). The primary goal of the residential school system was to assimilate Aboriginal people into the dominant Western society (Royal Commission Report on Aboriginal Peoples, 1996; Furniss, 1999). Aboriginal children were taken from their communities and robbed of their native language, culture, and customs (Ing) and Aboriginal parents and communities were deprived of their children. In the residential school system children were prohibited from speaking in their primary language and were harshly punished when they were caught doing so (Ing). Additionally, it has been well documented that many children suffered multiple forms of abuse (physical, sexual, emotional) from the staff working in the residential school system (Cull, 2006; Royal Commission Report on Aboriginal Peoples; Furniss). A number of residential schools were operating until nearly the end of the twentieth century (Ing; Smith, Varcoe, \& 
Edwards, 2005; Blackstock, Trocmé, \& Blackstock, 2004). For example, in British Columbia the last operating residential school closed down in 1984 (Greenwood \& De Leeuw). The residential school system has had lasting effects and has been associated with a number of negative outcomes including high suicide rates, sexual exploitation, substance use and abuse, poverty, low education attainment and chronic unemployment (Cull).

By the late $20^{\text {th }}$ century, pressures from human rights and Aboriginal activists resulted in the closure of the residential school system. However, in the midst of residential schools gradually closing down, provincial social workers were given authority from the federal government to enter Aboriginal peoples' homes on and off reserves and remove children from parents who did not meet the white middle class parenting standards of the dominant society (Fournier \& Crey, 1997). In 1947 the Canadian welfare council and the Canadian association of social workers argued that "Indian" children who are neglected lack the protection given under social legislation to white children (Fournier \& Crey). Subsequently in 1951 the Indian Act was amended under section 88 enforcing all provincial laws onto reserve land so long as they did not conflict with treaties or federal laws (Fournier \& Crey).

Child protection services for First Nations people became a provincial responsibility. However, the federal government negotiated separately with each province regarding the amount of money they would provide for "status Indian" children removed from their homes, as the federal government remained financially responsible for status First Nations people (Fournier \& Crey, 1997). There was a guaranteed payment for each "status Indian” child apprehended, meaning each provincial government received a payment from the federal government based on the number of children in government protective services. Consequently, a rapid increase in the number of Aboriginal children in government protective services ensued. In 1951 the percentage 
of Canadian First Nations children in government care was $1 \%$; however, by the end of the 1960s 30-40\% of the children in care were First Nations (Fournier \& Crey).

During this time period several social workers looking for employment found reserves to be easy targets to find work (Fournier \& Crey, 1997). "Social workers deprived of the information, skills, and resources to address the poverty, disempowerment, multi-generational grief and loss of parenting knowledge defaulted to a practice of mass removal..." (Blackstock et al., 2004, p. 903). Children were relocated from their parents, siblings and communities and sent far from their homes to stay with non-Aboriginal families (Cull, 2006). Additionally, social workers' caseloads were too heavy to allow for proper screening of foster homes, which largely resulted in countless children being placed in homes where they were abused and treated like slaves (Fournier \& Crey). The mass removal of children and youth that began in the sixties has been referred to as the "sixties scoop;" however it lasted far longer than a decade (Fournier \& Crey). Patrick Johnston developed the term "sixties scoop;" which generally refers to the period 1960-1980 (Sinclair, 2007). It is a term used to refer to the time period from the initial closing of the residential schools to the mid-1980s (Sinclair). Unfortunately to date there has been little difference in the situation of Aboriginal children and the child welfare system (Sinclair).

\section{Child Abuse and Neglect in Context}

Based on the 2003 Canadian Incidence study of Reported Child Abuse and Neglect, Aboriginal children represented $15 \%$ of the total number of substantiated ${ }^{3}$ child maltreatment cases. 10\% involved status First Nations children, $2 \%$ represented non-status First Nations children, 2\% involved Métis Children and 1\% involved Inuit Children (Trocmé et al., 2003). As

\footnotetext{
${ }^{3}$ The Canadian Incidence study considers a case substantiated if the balance of evidences indicates that abuse or neglect has occurred (Trocm $\square$ e et al., 2003).
} 
these statistics indicate, Aboriginal children and youth suffer a disproportionately higher rate of child abuse and neglect, which is primarily the result of systemic issues (Canadian Council of Provincial Child and Youth Advocates, 2010). There is no doubt that the history of colonial policies leading to family disruption has left a lasting impact on the parenting practices for many Aboriginal families (Sullivan \& Charles, 2010). One of the most prominent examples of family disruption was (as mentioned earlier) the residential school system. The residential school system prevented children from observing healthy parenting role models and subsequently many of the children that attended residential schools grew to be adults who were poorly equipped for parenting (Trocmé et al., 2004).

Child abuse and neglect is a serious problem affecting a number of Aboriginal families and communities, but it cannot be examined without considering the greater social, political and economic factors simultaneously occurring for a number of these parents and children (Canadian Council of Provincial Child and Youth Advocates, 2010). Aboriginal families in the child welfare system suffer tremendously high adversities (Trocmé et al., 2004). "Compared to Caucasian families, Aboriginal families have statistically significantly less stable housing, greater dependence on social assistance, younger parents, more parents who were maltreated as children, and higher rates of alcohol and drug abuse" (Trocmé et al., 2004 p. 594). These greater socio-economic factors have contributed to more Aboriginal than non-Aboriginal children being removed from their family and community following child welfare investigations (Trocmé et al., 2004). The child welfare system in Canada is complex; in the next section I present a brief explanation of the child protection system in British Columbia, to provide a general understanding of the bureaucratic structure of the province's child welfare system. 


\section{The Child Protection System in British Columbia}

In British Columbia (B.C.), The Child, Family and Community Service Act, is the legislation that directs the Ministry's Child Protection Services (Ministry of Child and Family Development, n.d.). The Ministry of Child and Family Development (MCFD or as it is commonly referred to, "the Ministry") is primarily responsible for managing the Child Protection services across BC (Pivot Legal Society, 2008). Currently BC's Child Protection services are organized into five regions. The Ministry of Child and Family Development's Minister delegates Directors in each region who then delegate certain authorities of The Child, Family, and Community Services Act to team leaders and child protection social workers throughout a number of Child Protection agencies across the province (Pivot Legal Society). To date, within the five regions there are 429 Ministry offices in the province as well as additional delegated Aboriginal agencies (Ministry of Child and Family Development, n.d.).

In $\mathrm{BC}$ there are also 22 Aboriginal agencies delegated by "the Ministry's" authority to carry out all or some of the legislative functions of The Child, Family, and Community Services Act. The scope of each Aboriginal agency's function is negotiated between the Ministry and the Aboriginal community that the agency serves (Ministry of Child and Family Development, n.d.). Aboriginal agencies' scope of authority ranges from voluntary services, recruitment and approval of foster homes to full Child Protection services. Of the 22 Aboriginal agencies, currently nine have the full authority of child protection delegations, which includes, if necessary, removing a child from a family or place (Ministry of Child and Family Development, n.d.).

Under The Child, Family and Community Services Act any person who has a reason to believe a child is in danger or may be abused, neglected or otherwise requiring protection, has a duty to report (Ministry of Child and Family Development, n.d.). Once a report has been made 
all reports are investigated by a child protection social worker. The child protection worker then must decide what the most appropriate and least disruptive action will be for the child, which could be any of the following outcomes: finding supportive services for the family; supervising how the child is cared for in the home; or if necessary, removal of the child from the family or place (Ministry of Child and Family Development, n.d.).

Child protection social workers do not all have the same level of authority ${ }^{4}$ and therefore decisions related to child protection investigations are generally not made by one isolated child protection worker (Pivot Legal Society, 2008). The process works in such a way that if a report is made, a child protection worker will investigate it, and then make the decision whether or not to get involved, and if the decision is made to get involved a family service file is opened (this is what is referred to later in the analysis as an "open file"). Once a mother has an "open file" she will have a child protection worker assigned to her case, who will follow the file until it is decided that the file can be closed. In the following subsection I move into the current situation for Aboriginal children involved in the child welfare system in BC.

\section{Current Issues Related to Aboriginal Children in State Care}

As of March 2010, statistics indicate that 54\% of children in government care in British Columbia are Aboriginal. From the available statistics it appears as though Aboriginal children are six times more likely than non-Aboriginal children to end up in government child protection services (Farris-Manning \& Foster, 2003). Since there is no national child welfare data system one cannot obtain the exact number of Aboriginal children in government protective services;

\footnotetext{
${ }^{4}$ As stated in the Pivot Legal Society Document (2008) social workers are usually divided into 4 groups: intake and investigations, these are the social workers that mostly do the investigations of reports; family services, these social workers are usually the social workers that supervise open files and are usually responsible for removals and more temporary removal situations; guardianship and Adoption Services, these social workers follow cases with children that are going to be in care more long-term and is called a continuing custody order; and lastly, resource teams are responsible for recruiting, approving and supporting foster placements.
} 
however, data collected by Canadian Incidence Study (CIS) indicated that in 1998 Aboriginal children made up $5 \%$ of the total population while making up $17 \%$ of the child population in care (National Collaboration Centre for Aboriginal Health, 2009-2010a). The current statistics indicate that approximately three times as many Aboriginal children are in government care today than the number of children attending residential schools at the peak of their operations (Blackstock \& Trocmé, 2005).

The removal of a child from the home, by law, is used as a last resort for children in unsafe situations and yet in many circumstances it is the primary intervention for Aboriginal families, as few preventative measures are used to try to maintain family relations (Cull, 2006; Palmer \& Cooke, 1996; Trocmé et al., 2004; National Collaborating Centre for Aboriginal Health, 2009-2010a). Separating children from the family can have extremely serious consequences for children, which is the reason it is used as a last resort (Cull). One of the key challenges is the nature of government intervention with Aboriginal mothers; Aboriginal women continue to live under the close watch of child protection agencies and are unfairly measured against the dominant Western society's ideals of what makes a good mother (Cull). The parenting styles of Aboriginal people often differ from those seen in middle class, Caucasian, nuclear families. Furthermore, because Aboriginal women are at an increased likelihood of living in poverty and heading single parent households (Greenwood \& De Leeuw, 2006), they are often at an increased risk of state intervention as "... [Aboriginal people] live their lives in a society that essentially makes poverty a 'quasi' crime and Aboriginal ethnicity a risk factor." (Cull, p. 147). Living in poverty is not an inherent Aboriginal quality but rather related to the outcome of issues such as unequal power relations between Aboriginal people and the state, colonial policies 
that have restricted Aboriginal peoples' access to land and resources, and ongoing racism operating throughout society, impacting employment.

Poverty, and other social status indicators such as employment rates for Canadian Aboriginal women, fall far behind those of other women (Browne \& Fiske, 2001). For many people living in poverty or below the poverty line, securing daily survival necessities like shelter, clothing and food is an ongoing challenge and concern. Attaining safe and adequate housing is a particularly complex issue for impoverished pregnant and parenting women and children. Poverty, as it relates to housing, is a complex problem that has particular effects on women and children (Duva \& Matezer, 2010). In Canada, housing issues, namely inadequate and insufficient availably of supportive housing, is a growing concern. Government cuts to social housing, which began in the 1980s, continue to impact certain groups' access to affordable housing (Carter \& Polevychok, 2004). Populations particularly impacted by the shortage of supportive housing include: First Nations people, people with mental illness and substance use issues, seniors, people with chronic illnesses, people with disabilities, women, children and people that are homeless (Carter \& Polevychok; Jategaonkar \& Ponic, 2011). One group particularly impacted by the shortage of social housing in Canada is female lone-parent households (Carter \& Polevychok). Cohen-Schlanger, Fitzpatrick, Hulchanski, \& Raphael (1995) surveyed social workers from Toronto's child welfare system to examine the relationship between housing problems and decisions around placing children into temporary care. "In $18.4 \%$ of the cases, the family's housing situation was identified by the family service worker ${ }^{5}$ as one of the factors that resulted in temporary placement of a child/children into care." (Cohen-Schlanger et al., p.554).

\footnotetext{
${ }^{5}$ The term family service worker refers to a "...professional social workers who visit families in their home as a routine practice, and have first-hand information of a family's housing circumstances. Therefore, these social workers are particularly knowledgeable about the major factors affecting the families and children they work with." (Chau et al., 2001, p.2)
} 
Similarly, one of the key findings from Chau, Fitzpatrick, Hulchanski, Leslie, \& Schatia indicated that in one out of five cases, housing was a factor that resulted in the temporary placement of a child into care (2001, p. 3). These findings are not meant to imply that adequate and affordable housing will directly result in the prevention of child apprehensions; rather, affordable, safe and accessible housing may help to decrease the number of children removed from homes, provide stability within a family's home environment, and decrease housing-related delays that factor in the return of children to families (Chau et al., p.9). The lack of social housing available in Canada intersects with the child welfare policies in a paradoxical manner, “...in that secure housing is often a condition of maintaining custody; yet having custody is a requirement of obtaining social housing." (Jategaonkar \& Ponic, p. 2). The housing crisis highlights one of the many challenges for many pregnant and parenting (or trying to parent) Aboriginal women living in poverty.

The government has been a key player in creating the negative stereotype of Aboriginal mothers. Aboriginal mothers are often perceived by the dominant western society as, "negligent, unfit parents." These negative stereotypes are seen as "cultural" characteristics, which perpetuates further interruption and interference into the parenting practices of Aboriginal women by the state (Browne, et al., 2005). Post-colonial feminist theory assists one in recognizing that socially constructed meanings of "culture" allow a new, more acceptable discriminatory discourse in which certain traits are considered "cultural." From this 'culturalist' perspective, "culture" is viewed as a neutral, static concept that does not consider how history, economics, policy and social status affect and shape many peoples' lives (Browne \& Varcoe, 2006). The "cultural" characteristic of Aboriginal mothers, viewed as being "neglectful," not only misrepresents many Aboriginal mothers but also obscures the underlying root causes of 
why some Aboriginal women are unable to adequately provide shelter and resources for their children, which include issues such as, poverty, trauma, violence, social exclusion and discrimination.

\section{Health, Healthcare, and Aboriginal Women and Children}

According to the British Columbia's Provincial Health Officer's Report (2009) the health of infants and children is commonly accepted as an indicator of the overall health and well-being of a population. Although the statistics have improved in the last couple decades, in British Columbia Aboriginal populations continue to have significantly higher rates of infant mortality, neonatal mortality, post-natal mortality, prematurity, low infant birth weight and high infant birth weights, all of which are used to measure the health status of a population (Provincial Health Officer's Report). Another indicator of the overall health of a nation relates to reproductive health. Trends such as teen pregnancy, preterm birth, and birth rates all shed light on the health status of women and children (Provincial Health Officer's Report). In British Columbia, Aboriginal women continue to have significantly higher rates of teenage pregnancies and overall higher birth rates than non-Aboriginal women.

Healthcare access for Aboriginal women is known to be highly problematic, and shaped by racism, discrimination, and stereotypical thinking (Browne, 2007). In particular it is well established that Aboriginal women are late to access prenatal care or avoid prenatal care entirely and receive inadequate prenatal care (Heaman, Green, Newburn-Cook, Elliot, \& Helewa, 2007; Heaman, Gupton \&Moffatt, 2005), and this healthcare access issue also has been suggested as related to ongoing colonial and discriminatory practices (Benoit, Carroll, \& Westfall, 2007; Brown, Varcoe \& Calam, 2011). 
There is little available within the literature examining how the threat of child apprehension impacts the experiences of Aboriginal women's decisions or ability to access healthcare services. Thus this study explored Aboriginal women's experiences accessing healthcare services when child apprehension is a threat, to gain a better understanding of these potentially vulnerable interactions between Aboriginal women and healthcare. The overarching goal of this study was to learn how to improve the quality and safety of healthcare delivery to Aboriginal women and children. 


\section{CHAPTER 3: METHODS}

\section{Introduction}

This chapter will describe the research methods; it includes the research plan, which occurred in two phases and concludes with the ethical considerations. The study utilized exploratory qualitative research methods, following the principles of ethnographic research.

Ethnographic methodology was chosen for this study as it examines a number of human experiences including beliefs, relationship patterns, and ways of living (Thorne, 2000). Ethnographic methodology aims to enhance understanding of how people experience their world and their working environment (Roberts, 2009).

\section{Research Plan}

The study was conducted in two phases and was part of a larger study examining primary health care (PHC) service delivery to Aboriginal and non-Aboriginal women and men at two Urban Aboriginal Health Centres (the larger study will herein be referred to as the UAHC study). ${ }^{6}$ The key modes of data collection in the UAHC study included: participant observation, shadowing of staff members, interviews with staff members, and interviews with patients as well as focus groups with patients. Seven in-depth interview transcripts and accompanying fieldnotes were used for phase 1 of the study, which is described in the following section. The goal of this phase was primarily to synthesize some foundational themes and use those themes to modify the interview questions for the second phase of the data collection (See Appendix A and Appendix B).

\footnotetext{
${ }^{6}$ The co-Principal investigators on this study include: Browne, Varcoe, Lavoie, Wong \& Smye. The UAHC study is conducted in partnership with two indigenous PHC centres: one in a large urban centre, and one in a smaller regional city. Both of the centres aim to serve Aboriginal and non-Aboriginal people who are most affected by poverty, historical trauma, social exclusion, racialization and discrimination, by incorporating a model of service that includes indigenous approaches to health and healing (Browne et al., 2007). The key modes of data collection in the UAHC study include: participant observation, shadowing of staff members, interviews with staff members, and interviews with patients as well as focus groups with patients. This study has undergone external review by CIHR and UBC REB.
} 


\section{Phase One: Analysis of Existing Data Set}

\section{Data Collection}

Phase One of this study involved an analysis of transcripts of in-depth interviews previously collected for the larger UAHC study. The analysis included interview data from women participants $(n=3)$ and health care provider participants $(n=4)$ as well as accompanying fieldnotes.

\section{The Sample}

The interviews selected for Phase One were women and health care provider in-depth interview transcripts from the UAHC study that addressed Aboriginal women's experiences with healthcare services when child apprehension of their children either occurred or was threatened by child protection services. The inclusion criteria for the women participants' interview transcripts were that they self-identified as Aboriginal and that they spoke of at least one experience of having either a threat of child apprehension or actually had their children removed from their care by a child protection worker. The inclusion criteria for the health care provider interview transcripts were that they spoke of experiences working with women that have either had their children removed from their care or have had it threatened by a child protection worker. The Women Participants

The three women participants all had experiences of child apprehension investigations by child protection social workers [the women regularly referred to child protection social workers or the Ministry of Child and Family Development (MCFD) as 'the Ministry']. All of the women had one or more of their children removed from their care on more than one occasion. The three women all self-identified as Status First Nations Aboriginal women, with ages ranging from 3234 years. None of the women had paid employment at the time of the interview. Data were not 
collected regarding income status; however the women described their monthly income as ranging from no income to $\$ 975.00$ per month. None of the women explicitly stated having any issues with housing at the time of their interview, although one woman was staying in a shelter, as she had just left her partner after being assaulted by him and sustaining a face injury.

\section{The Health Care Provider Participants}

The four health care providers all had extensive experience working with Aboriginal communities and with 'marginalized' populations ${ }^{7}$. The health care provider participants' ranged in years worked in healthcare from 24-30 years, and in their current place of employment for more than 2 years. Three of the health care provider participants' were working as registered nurses, and one as a social worker at the time of their interview. All of the health care provider participants were women, ranging in age from 50-64 years old. One of the health care provider participants self-identified as a Status, First Nations, while the three other participants all reported their ethnicity as stemming from Western European backgrounds.

\section{Data Analysis}

The primary method of data analysis used in this study was thematic analysis. Thematic analysis, like all forms of analysis, is a way of seeing what is being observed (Boyatzis, 1998). The purpose of this analysis is to recognize an important moment, then encode that moment and interpret what is being observed (Boyatzis). The focus is on coding data into themes and categories and thus began in the early stages of analysis.

I first used the NVivo software for coding and organizing data sets. Once data was organized and categorized, I followed the principles of thematic interpretative analysis based on the procedures of interpretive description (Thorne, 2008) to interpret meaning in the data and

\footnotetext{
${ }^{7}$ The term marginalization or marginalized populations is difficult to define but in this paper marginalization refers to "... a complex socio-historical process...those most in need, not already able to meet their own needs, with limited access to resources or who exist outside of power structures.” (Laverack \& Labonte, 2000, p. 259).
} 
gain new knowledge. The main goal of this Phase One was to determine some initial themes and use those themes and insights to modify the interview guides developed for Phase Two. The first theme that developed in this analysis was the context of the women's lives, which were all impacted by poverty, violence and substance use. The context of the women's lives was discussed in-depth within the interview transcripts, which made it the starting point for analysis. Later in the analysis process, new themes which were not anticipated developed; these included a lack of available services for parenting or wanting-to-parent fathers and partners and the shared value amongst the participants of their drug and alcohol counselors.

\section{Phase Two: Additional Primary Data}

In the second phase of data collection, in-depth face-to-face interviews were conducted. Ethnographic research commonly uses interviews as a means of data collection. The aim of the ethnographic interview is to learn the social and cultural meanings that exist within a social group. The interview focuses on interactions as well as the social context and the social construction of reality (Sorrell \& Redmond, 1995). Unlike in quantitative research, where the researcher aims for objective data, interview data conveys respondents' thoughts, opinions, and impressions (Carspecken, 1996; Sorrell \& Redmond, 1995).

In ethnographic interviews it is common to begin the interview with some open-ended questions and then follow with more specific questions for clarification purposes or to further examine emerging themes (Fetterman, 1998). The interviews carried out in Phase Two were indepth, semi-structured, and followed a general outline with some prepared questions; however, as the interviewer I was flexible towards potential changes in the direction of the interview (Sorrell \& Redmond). As the facilitator, I was committed to creating a safe environment and thus all of the interviews were carried out in a private room at either of the centres used for participant 
recruitment. All of the participants were familiar with the environment, as the participants were either clients or employees of their respective site. At the beginning of each interview the participants were informed verbally as well as on the consent form of their rights to 1) refuse to participate, 2) refuse to answer any specific questions, 3) to leave the interview, and 4) to ask any questions of the researcher(s) at any time during the interview or during the research process itself.

\section{Data Collection}

\section{Study Sites}

The study was conducted through two Sites. Site A is a Primary Health Care (PHC) clinic located in northern British Columbia. Site A is committed to providing services that are accessible and culturally safe for marginalized Aboriginal and non-Aboriginal people, and informed by indigenous approaches and understandings of the root causes of health and illness. In relation to the prenatal care, during the period of data collection Site A had four registered nurses (RNs) as well as one nurse practitioner employed; three of those RNs do the bulk of the prenatal and maternal care.

As one of the health care provider participants explained, the nurses at Site A work as case managers or key workers for the prenatal clients. Approximately eight years ago a nurse from Site A who has been at the clinic since its inception created a program within Site A called "Breaking the cycle." The goal of this program is to prevent children from being removed from their mothers or families by supporting women during and long after their pregnancy through a 'wrap-around approach.' Additionally, within the health clinic a group of health care providers created what they refer to as the psycho-social team. The psycho-social team consists of nurses, a nurse practitioner, drug and alcohol counselors, social workers and an elder. This psycho-social 
team spends a significant amount of time with the pregnant and parenting women clients, and as a team, the members work collaboratively on "Breaking the cycle." Site A utilizes a number of external community services (food banks, transition homes, and other social assistance programs) to help enable women to gain the resources necessary to safely care for their children.

Site B is located in an urban area; it is a program (versus a health clinic or health centre) that provides services to pregnant and parenting women from its surrounding area. Site B's mandate is that the women must have either a current or past issues with drugs or alcohol. The mandate is that they will keep a woman as a client until the time her child turns 18 months of age or less depending on the client's needs; however, when necessary, the program is flexible around keeping a client longer than the mandated 18 months. Site B offers medical care by community health nurses and physicians (e.g., prenatal care, prenatal education), in addition to a number of other services, which include: social work, peer support, outreach work, drug and alcohol counseling, food services (Monday- Friday there a hot lunch served and once a week the pregnant women receive a food bag), legal-aid, financial aid, infant development consulting, and a nutritionist. The service providers have varying schedules (meaning not all of the services are provided Monday-Friday) but all of the services previously discussed are available in-house and all of the medical services are available Monday-Friday. At the time of the study approximately $70 \%$ of the women that access Site B are Aboriginal, and since the program's existence the percentage of Aboriginal clients has relatively stayed constant.

\section{Participant Recruitment}

The participant recruitment method was a combination of word of mouth, personal contact and snowball sampling at the two Sites. The inclusion criteria for the women participants were emancipated women over the age of 14 that identify as Aboriginal, and have experienced 
the threat of apprehension of their children. Some of the women participants were approached by health care providers from the respective Site they access. Others heard about the study through snowball sampling, as a number of the women had friends or family that had experiences with the child protection system. Once the women heard about the study, they were given the research teams' contact information, which was left at both of the centres. Potential participants were given more information on the study through telephone conversations or in face-to-face interaction. To ensure informed consent, the entire consent form was read out to the women participants unless they explicitly requested otherwise. This was done to insure that the information was communicated regardless of the woman's literacy level.

The inclusion criteria for health care providers were that they had extensive experience working with women who have had state apprehension of their children threatened. I was given an opportunity to present the study personally at Site A at a team meeting and at Site B, I fully informed one health care provider about the study, which she then presented at a team meeting. Both Sites felt this study would be beneficial for the clients they serve and therefore a number of staff members were interested in contributing their knowledge and expertise.

\section{The Sample}

\section{The Women}

A total of nine women participants were interviewed from the two Sites. Of the nine women participants five were from Site A and four were from Site B. All of the women selfidentified as Aboriginal: seven self-identified as Status First Nations; one woman as non-status First Nations; and one woman as Metis. The women ranged in age from 28-49. All of the women participants had more than one experience of having their child or children apprehended by a child protection social worker. At the time of the interview two women were living in shelters, 
one in supportive housing and the remaining six in either an apartment or duplex house. None of the women were employed at the time of their interview. I removed one of the women participant's interview transcripts from the study, as it became evident once the interview began that the participant was not fully informed about the study. Because she did not want to talk very much about the focus of the study and because she did not seem to understand most of the questions, I was not confident that she had truly provided informed consent.

\section{The Health Care Providers}

Eight health care providers participated in this study. Four of the health care providers were from Site A and four from Site B. All of the health care providers had experience working with pregnant and parenting women who have experienced the threat or actually had their children apprehended by a child protection social worker. The health care provider participants ranged in years worked in health care from 5-22 years, working with this particular population (women at risk or women that have experienced child apprehension by the child protection workers) from 1-17 years. The health care providers included: two registered nurses, one social worker, two drug and alcohol counselors, a physician, a peer support worker and an outreach worker. All of the health care provider participants were female, ranging in age from 35 to 65 years old. Three of the health care providers identified their ethnicity as status First Nations, two as Caucasian, two as European- Canadian and one as Japanese.

I interviewed all of the participants once. The interviews ranged in time from twenty minutes to one hour. In my proposal I stated that the interviews would take approximately thirty to ninety minutes and the majority of the interviews lasted one hour so this left ample time for each participant to share their experiences. Initially it was proposed that I would also do participant observation in the form of "shadowing" the health care providers at the health care 
provider's clinic or during a home visit. However, it was decided by the health care providers that this would not be appropriate for the women given the vulnerability and sensitivity of the subject. Thus the primary form of data collection was interview transcripts as well as fieldnotes written following each interview. With the permission of each participant all of the interviews were audio recorded.

\section{Data Analysis}

The process of analysis began early in the data collection phase and also followed the general principles of thematic analysis. Similarly to Phase One, data was first categorized into broad codes, which were then further analyzed and developed. The initial broad codes were used to draft an outline of the findings. As the process of analysis progressed, new linkages developed within the data. Initial codes were those findings that were commonly described by the participants, which included the socio-economic and historical context of the women's lives; personal experiences with the child protection system; and healthcare service experiences. Once these broad codes were outlined, a more detailed analysis ensued. In the later stages of analysis broad themes were broken down into subthemes. For example, the socio-political and historical contexts of the women's lives were shaped primarily by: ongoing disruption of Aboriginal families and communities; structural inequities and the context of poverty; and the context of violence and abuse.

\section{Ethical Considerations}

Ethical approval was obtained through the University of British Columbia's Behavioural Research Ethics board (see preface).

Confidentiality of data was maintained at all times. Anonymity was maintained by assigning all participants with code numbers that correspond with interview transcripts. The data 
was stored in password-protected computerized files. Audio-tapings, flash drives and hard copies were all stored in a locked filing cupboard at UBC School of Nursing in co-supervisors Varcoe and Browne's workspace.

The interviews in Phase Two were conducted in a safe, private and convenient location agreed upon by both the participant and researcher. Informed consent was obtained prior to conducting the interview (see Appendix C and Appendix D). The informed consent included a request to audio-record; a written and verbal explanation regarding audio-recording was made at the beginning of the interview. The participants were all notified that audio-recording could be refused, and discontinued or erased at any point during the interview. The participants were also informed that at any point they could choose to stop or leave the study or interview. Participants were be given the option on the informed consent form to have a one page summary of the findings mailed to them.

The Co-Supervisors Varcoe and Browne have extensive experience conducting research in partnership with Aboriginal people and Aboriginal communities. Because the study involved interviewing some Aboriginal women and asking questions about their experiences with the threat of having their child or children apprehended, the seriousness of the potential of bringing up a traumatic experience for these women was recognized and therefore consideration for the sensitivity of this study was taken at all times. For instance, the participants were informed verbally and on the consent form about their right to stop the interview, refuse to participate, leave the interview at any time, and to ask any questions at any time during the interview process. Furthermore, should the participants have required it, psychological counseling was available through a list of available services in the participant's community. Consideration was also made in the case of the interview bringing forth a traumatic experience that required 
immediate intervention, which included stopping the interview, and taking the participant directly to a trained counselor. Both Sites used for participant recruitment have trained psychological counselors on service. A potential benefit for women participating in this study was thought to be that for some women, this may be one of the few chances they have had to speak freely and confidentially about their experiences. 


\section{CHAPTER 4: PHASE ONE FINDINGS}

\section{Introduction}

In this chapter I present the findings from Phase One. The findings have been organized into six main sections: the first three sections convey the main socio-economic challenges experienced by the women participants, which included poverty, violence and substance use. All of the women interviewed had a number of concurrent socio-economic issues impacting their lives (e.g., poverty, substance use, violence, chronic pain, or trauma) and from the health care providers' perspectives, most women at risk of having their child or children removed by child protection social workers are dealing with similar socio-economic issues. Next is an analysis of the involvement of fathers and partners in their roles as parents, followed by an analysis of how the threat of child apprehension impacted the women's experiences accessing healthcare services. This chapter concludes with a discussion regarding follow-up.

\section{Socio-Economic Factors Influencing Women's Lives \\ Poverty and Structural Inequities}

Stated by Wharf, “... [T] he single most powerful way to improve child welfare is to eliminate poverty among children and families... The fact that poor families, particularly led by single women, are substantially overrepresented in the child welfare enterprise has been documented in a series of studies..." (2007, p. 229). Furthermore, there is mounting evidence that links poverty to poor physical and mental health (National Council of Welfare Reports, 2001, p. 8; Canadian Institute for Health Information, 2008b; Reutter, Veenstra, Stewart, Raphael, Love, Makwarimba, \& McMurray, 2006). Although poverty rates in Canada overall are on the decline, subgroups within Canada continue to experience much higher rates. These subgroups include single parent families, urban Aboriginal peoples, single female seniors, and 
recent immigrants (Reutter et al.). This corresponds with the health care provider participants' perspectives; all highlighted that women at risk of having their child or children removed by child protection services often belong to the subgroups of people that experience far higher rates of poverty, particularly single parent families and Aboriginal peoples.

Although only one woman explicitly expressed a current challenge related to money at the time of her interview, all of the women described related challenges, including issues with transportation, food or clothing. In the following quotation, one woman explained her need for assistance with transportation, a concern reiterated by all of the women. In this excerpt, she explains how health care providers from the clinic she attends assist her with transportation: [Name of health care provider at the clinic] drives me way out to get food from the Salvation Army... because I always have my baby with me so it is hard for me to get around and load all of this food, so [the health care providers] always take time out to give me a quick ride from $\mathrm{A}$ to $\mathrm{B}$, which is always really helpful. (P13)

This is echoed in the following quotation, as the woman explained what services provided at the clinic she found helpful: “...[T]hey pay for your trip [to the city] and back home and they'll pay for your food but you have to stay in town for longer than a day." (P11)

All health care provider participants indicated that most women who have had investigations with child protection services experience poverty. One woman participant spoke explicitly about her how important it is for healthcare services and providers to understand poverty:

And that's what I like about this place, because I'm like that, I know what it's like to be out there and down and out and not have a home and not have nothing but your drug of choice... and a lot of the people I see [at the health clinic] are my friends, like I know 
them from when I was on the street. So when I come here with my baby, they all say hi to me, everybody, it's like a warm place. Like there's no judging here, it's a safe environment. (P13)

This woman further described how beneficial it is for her to come to a health clinic that understands her circumstances:

...I've come [to this health clinic], there have been a couple of times that I've run low on diaper wipes and [the staff at the health clinic] has given me diaper wipes. Just little things that make you feel really, one thing I've noticed is that they really understand poverty. Like they understand that people are in poverty and people are in need of more places like this, and more housing and more drop-in places for people like [pause] myself. (P13)

Similarly, all health care provider participants expressed in their interviews how important it is for healthcare to include services that address the social determinants of health, in order for women to live a lifestyle that a child protection social worker would see as healthy and safe enough that they could maintain custody of their children. From these health care providers' perspectives, women need secure and adequate income, safe housing, food, clothing and a means of transportation to be able to parent successfully. Housing is a particular challenge, as in Canada a mother's home is used in the assessment of her competence to parent (Lessa, 2002).

All of the women expressed how much they value the assistance they receive from the health clinic with which they are connected, particularly in relation to transportation. This included having health care providers make home visits, as well as bus passes or rides to appointments and community programs such as food banks. Thus poverty appears to be a complex socio-economic issue that shapes women's ability to maintain custody of their children. 


\section{Substance Use}

All of the women interviewed in Phase One either currently or in their past have experienced challenges with substance use. The women most often described using alcohol during their pregnancies, but also mentioned other substances such as Tylenol 3 and crack cocaine. The health care providers said that substance use is one of the main reasons women lose custody of their children. One woman participant stated that she was not using drugs or alcohol at the time of her interview; one said she was currently using Tylenol 3, as she was experiencing pain from an ongoing health issue; and another said she was trying to stop using alcohol. The women participants all stated that they had stopped and restarted using drugs or alcohol at least once during the time they were pregnant or parenting. All of the women said that in order to be successful in getting off drugs or alcohol, they required a significant amount of support, particularly from their drug and alcohol counselors.

The women participants all spoke about how, as women known to have issues with drugs or alcohol, they truly appreciated healthcare services that were delivered respectfully and nonjudgmentally. The women valued being treated with respect by the health care providers at the clinics they access; however, all of them also described experiencing significant discrimination, particularly in the hospital setting.

In addition to tangible supports such as transportation and help with access to food and shelter, what particularly made healthcare services most valuable for the three women in Phase One was support in dealing with child protection personnel. For example, one participant stated: Yes, because there was a time when the welfare, it really started to bend on me. Like yesterday I just about had a nervous breakdown and boom I made one phone call and I wanted to just give up and let [“The Ministry"] have my baby. And I just wanted to just 
use [drugs] and it just all came back, like why didn't I just stay the bad person that I was, right... I phoned [a staff member at the clinic] and a drug and alcohol counselor and The Ministry was at my room. So I phoned [staff at the health clinic] they came up right away, and [two staff members from the clinic] came up, and they really supported me through it and The Ministry was there to apprehend my baby from me. And [the staff from the clinic] stopped them, and my baby got placed now with my sister- in-law... (P13)

Both health clinics where the health care provider participants worked take a harm reduction approach to pregnant or parenting women dealing with substance use issues; however, from what is described in the health care provider interviews, it appears that many MCFD social workers expect known "substance-abusing" mothers to be completely off drugs and alcohol if they want to keep or regain custody of their children. This became a question for follow up in Phase Two of the research. In particular, missing from the Phase One data set was information on whether services are available for parenting women who begin using drugs or alcohol to a degree that would impact their children's safety. More specifically, when mothers are in crisis or using drugs or alcohol, are there safe places they can send their children that would not necessarily result in a child being removed? All of the women interviewed talked about the substantial progress they had made towards a healthier lifestyle that was often, but not all of the time, safe enough for them to have custody of their children. Thus a useful follow-up question for the second phase of this study was determining whether there are supportive services for mothers in an emergency situation. The women interviewed were all well connected with supports from their health clinic and, with assistance from health care providers, able to regain at least partial custody of their children. 
Interestingly, two health care providers explained changes in the way they are now working with MCFD; one described how over the years she has actually created a better working relationship. As a result, her team has become extremely successful in preventing unnecessary child apprehensions.

So we really have the right heart and the right mind and we focus on the here and now, and the future, and the future of the children. So a pregnant mom comes in, that's the future. And I put all my effort in, all of it, all I've got. And with great results because we now have very respectful relationships with [MCFD] and so [MCFD] sends all their clients who they know are pregnant or who are in jeopardy of losing the children to care or are struggling to get them back, they send them... if they identify as Aboriginal. (HCP12)

The health care provider participant interviews indicate that if a mother has a crisis (for example, she starts using drugs or alcohol again) and does not have health care providers or some other support system present, she can quite easily lose custody of her children. One woman from Phase One described how when she starts going on a "downward turn" she instantly contemplates just giving up her children to MCFD, because that is what she assumes will be the end result anyway. Thus a follow-up question for Phase Two was: What have women done in the past when they went back to using drugs or alcohol? And a further follow-up question: What services would be helpful for a woman in a crisis who is considering using drugs or alcohol?

The health care providers and women from Phase One agreed that women with substance use issues truly value healthcare services that assist and advocate for women to gain or maintain guardianship of their children, through empowering and supporting them to live a lifestyle that is healthy and safe for their children. For example, one health care provider stated: 
We work together on plans and how we can keep them safe and work towards whatever they're capable of working towards - small bites, small steps. But ultimately this is a success, I think we have a ninety-seven percent rate that people either can hold onto their children that they're not apprehended, or, if they're apprehended get them back within one and a half to two and a half years. (HCP12)

All of the health care providers reported that their health clinics have strong support systems for women struggling with substance use and other issues such as violence and poverty, and that it is these ongoing supports that can help mothers maintain some type of guardianship of their children. However, the health care providers and women participants all stated that more services are needed for both pregnant and parenting women with complex socio-economic challenges. All participants mentioned how high the workload is for the clinic staff at both sites. The women participants appear to value having a drug and alcohol counselor, but the general consensus was that more counselors were needed, including general counselors (such as sexual assault or grief and loss counselors). Therefore another question to ask in Phase Two was: What is it about drug and alcohol counseling that is so beneficial? This analysis also suggested that pregnancy is often a motivator for women to get off drugs or alcohol and an opportunity to link them into healthcare services.

\section{Violence}

Throughout the data in this analysis, and consistent with what is evident in the literature, violence and abuse appear to be extremely complex issues for pregnant and parenting women. One health care provider participant stated that a violent or abusive partner or home environment is one of the main reasons children are apprehended. This same health care provider stated that virtually all of the women her health clinic sees have a history of some kind of abuse, mostly 
sexual abuse. Health care providers find that many women who have experienced violence and abuse lack trust in other people, including, and possibly particularly, health care providers. The approach the health care provider participants use and believe to be most successful is "not forcing" anything on the women and, if women choose not to undergo certain treatments, they are not judged.

Interestingly, at one of the health clinics, a woman can stay in the program even if she is currently with a violent partner; however, the health care providers suggested that from MCFD's perspective, remaining in a violent relationship is problematic if a woman wants to maintain guardianship of her children. The majority of the health care providers and one of the women from Phase One described how difficult it can be for many women to leave a violent or abusive partner, and said that having children makes it much harder to leave. One health care provider talked openly about the complexities of leaving a violent partner. For many women, she said, it is quite challenging to break all connections with the violent partner even if they want to leave. This is particularly true for Aboriginal women, as often the woman is connected to the man in multiple ways (perhaps he is from her community and nation or related to someone in the family). Therefore, even if the woman leaves the relationship, she must still see the partner. Furthermore, if the partner is the father of the child, often the woman wants to include him in the child's life. This is significant with regard to delivering care to women in violent relationships, as often a woman is required to leave the relationship if she wants to maintain guardianship of her children. Thus I foresee the decision to utilize healthcare services to be a complex choice for women in violent relationships, as it may come with the expectation that she should leave a relationship that she may not be able to leave at that time. 
In fact, one woman participant spoke directly about how hard it is to permanently leave a violent relationship:

Yeah, it's weird for me because I've come from nothing but abusive relationships and I actually did a dumb thing. I was missing the chaos and stuff so I phoned my ex and some stuff happened, I had to let go again, but yeah, [be]cause I was missing the chaos. And then I got, no I've got all these cravings and, but it's I'm getting through it, I mean it's not easy, but I get through it. It's one day at a time, one second at a time actually. (P 11)

One health care provider emphasized that violence and abuse must be addressed as part of the healthcare these women receive, primarily by ensuring that women who are in an abusive or violent relationship have a safety plan, a safe place they can go, and the means to get there. Lastly, one health care provider expressed that she thought some women find it even more difficult to leave violent relationships if they were in foster care themselves, because they have a vision of having their own family, which includes their children as well as the dad. Thus some women will stay in a violent, unsafe relationship just to hold on to that vision of the complete family with both a mother and a father.

As the previous sections highlight, for many women it is the context of their lives that impacts their parenting experiences. Thus if the healthcare system aims to keep families together, it must address the greater social-economic conditions of pregnant and parenting women. The women interviewed for this study all shared stories illustrating how much they depended on and appreciated the supports available through the clinics they attend, including help with transportation, outreach, counseling, food, and assistance with legal aid. 


\section{Fathers and Partners}

There appears to be a gap within the services aimed at supporting pregnant and parenting women involved with MCFD and this relates to services available for fathers or partners. There seems to be a need within the healthcare system to incorporate fathers or partners into the treatment for pregnant or parenting women, as this health care provider explained:

Ideally we would have something that would include the men because as bad as some of these guys are they're not throwaway people; you have to see value in them too. And they're from all screwed up backgrounds too and who knows what they need but they need something. And I think especially in the Aboriginal communities with the history of colonialization and all the bad effects and all the fallout from that. You see men who are stripped of their purpose in life, you know, being able to carry out their role in the community. (HCP5)

As mentioned earlier, one of the clinics welcomes partners to be involved in the pregnant or parenting women's treatment, but it appears that this program is unique, particularly in cases where the partner is seen by MCFD as posing a threat to the child or children (e.g., the partner is violent or has a criminal record). However, at this clinic the woman has the authority to choose whether or not the partner is involved and can change her decision at any time, as this health care provider explained:

And the other thing about the program is it's not women only. If she comes in with a partner then he's welcome too. And a lot of the other programs exclude men so we want to involve the partners because that's an important support for her... The partners are welcome but if she tells us that they're fighting or something and she doesn't want [him] here then we support that and we will exclude the partner. And sometimes that changes 
on a weekly basis, daily basis, yeah. And that's another reason why it's important for us to have our team meetings so that we can all be on the same page around what's going on with the women, who's their support, who do we need to let in, who do we need to keep out? (HCP5)

From this health care provider's perspective, many male partners depend on the woman to take care of them. Even if the man is abusive or heavily using drugs or alcohol, many women would stay with him because they do not want to leave the partner with no support for himself.

The Ministry will assess the partner too if he's in the picture and often they have criminal records or they have substance use, they're not stable so they are seen as presenting a danger to the baby in the home. So sometimes the women are told "you can go with the baby and you can live here but he can't be involved." But again sometimes the women really want him involved especially if he's the father of the baby because they want to have a family. And so we see women who grieve so much over that situation and sometimes the women are not willing to leave the man ... And it's in [MCFD's] supervision order that they are not to have contact with that guy because he's a danger. And sometimes the woman will go out the next day and meet with him, with the baby, and then they end up losing the baby. (HCP 5)

It is also apparent from this healthcare provider's interview that there is very little (if any) support for fathers and partners who are trying to be part of a family but are struggling with their own issues of anger, violence or substance use. Additionally, from this health care provider's perspective, the men should be involved because the women often want them to be. Therefore in Phase Two I asked additional questions about how partners could be better served when they are dealing with issues such as anger and violence. Is there an approach that could better incorporate 
partners while maintaining the safety of the women and children? Although this was not originally the focus of this study, it has become apparent that the role of fathers and partners needs to be further explored, as there appear to be few parenting services available to help these men deal with their own anger, violence and addiction.

\section{Accessing Healthcare Services When Child Apprehension is a Threat}

There was little data that indicated explicitly how pregnant or parenting women's decision to access healthcare services is impacted by investigations or experiences with child protection services; however, this analysis highlights how complex it is for many women living with multiple socio-economic issues to maintain a lifestyle that is safe and healthy enough to maintain custody of children. Two health care providers spoke about some women's negative experiences with child apprehension, as this health care provider explains:

And we've had women, we've had women who have had multiple babies removed by The Ministry and then they're able to, with enough support, they are able to succeed and do really well. And so we never give up hope, even if she has six kids in care because sometimes if you look back in her history, the reasons for removal were, seem very unjust to us. Like she was sixteen years old and she didn't know what to do or she had a violent partner and instead of taking mom and baby to a safe place, they take the baby away. (HCP 5)

However, one health care provider stated that some women will avoid clinics where the program's mandate is to provide services to pregnant and parenting women with a past or present substance abuse issue, as they see this as increasing their risk of having a child apprehended:

[C]oming [to this centre] is a real double-edged sword for women. They get great support but they also kind of get outed because anyone whose associated with [this 
centre] is seen as someone who is a drug addict and there are a lot of people out there, professionals who don't understand the clients we have and they just assume that everyone who comes [here] is street-entrenched, addicted, not a suitable mother. So over the years we have to educate and re-educate and constantly be enlightening other agencies and other people about who the women are that come here... So what we encourage the women to do is to do an early referral to The Ministry...we try and get women hooked up with supports early on and get enough documented information about how well they're doing and the good changes they've made so that by the time their baby is born they have a lot of good stuff documented... (HCP5)

The process of the early referral was further explored in Phase Two of the study, as health care providers from both study sites suggested contacting MCFD during pregnancy. This health care provider said that many women do not want to be referred to MCFD because they have been told by other women that once MCFD is contacted, the women are more likely to have their children apprehended since they are now a "known drug user," increasing the likelihood of having their children removed.

None of the women talked explicitly about how the threat or perceived threat of child apprehension impacted their decision to access healthcare services. Two women emphasized their frustrations with MCFD, and one described how she felt quite poorly treated by a social worker from MCFD, believing that MCFD did not respect her "rights." Another woman was upset because her child protection social worker was not available to come back and review her current situation so that she could regain custody of her children. However, overall there was no data that explicitly stated how or if child apprehension investigations or threats by MCFD impact 
women's decisions or experiences accessing healthcare services. This was therefore a focus of the interview questions in Phase Two.

\section{Conclusion: The Basis for Follow-up Questions for Phase Two}

The women and health care provider participants presented the common challenges that pregnant and parenting women involved in the child protection system experience. However, this analysis provided little understanding about if or how the threat of child apprehension impacts Aboriginal women's decision to access healthcare services. Therefore the interviews in Phase Two focused more specifically on whether the threat of child apprehension impacts one's decisions or experiences accessing healthcare services. Additionally, Phase One raised the question of what services are available for partners or fathers. There is no clear picture as to how best to include partners in the care of pregnant women struggling with drug and alcohol use, particularly if the partner is violent. Yet there is a real sense that these men also need help. Therefore in the second phase of this research, the issue of fathers and partners was further explored. A better understanding is also needed to learn what supportive services are available for parents in a "crisis" moment. Lastly, because all of the women found their drug and alcohol counselor to be beneficial to their health and well-being, I aimed to better understand what it is about a drug and alcohol counselor that is seen as beneficial. 


\section{CHAPTER 5: PHASE TWO FINDINGS}

\section{Introduction}

The findings presented in this chapter were developed by analyzing the additional primary interview data collected in Phase Two. They have been organized into five main topics: the socio-political and historical context of the women's lives; the bureaucratic structures governing child protection services; Aboriginal women's experiences with child protection services; healthcare access in context; and, lastly, setting up for success while navigating a complex system. I begin the findings with the socio-political and historical context of the women's lives. The findings here indicate that the context of the women participants' lives were quite similar to the socio-economic challenges experienced by the women participants from Phase One

\section{Socio-Political and Historical Context of Women's Lives}

The health care provider participants identified common challenges they see faced by pregnant or parenting women who are likely to be involved with the child protection system. They included: being a single parent or having a partner the women cannot depend on; a history of being in foster care themselves; a general lack of family or community support; living at or far below the poverty line; homelessness or struggling to find safe and secure housing; ongoing abuse and/or a history of abuse and violence (sexual and physical); trauma issues; mental illnesses or cognitive impairments; drug and alcohol abuse issues; and low educational levels

(for example, not having completed a high school degree). These challenges were echoed in the women participants' interview. In fact, most women were facing several of these issues. In the following section I organized these challenges into three sub-themes: ongoing disruption of Aboriginal families and communities; structural poverty; and violence and abuse. 


\section{Ongoing Disruption of Aboriginal Families and Communities}

As stated in the literature review, disruption within Aboriginal families and communities by government agencies and colonial policies is not new. Aboriginal family and community disruption was the result of oppressive government policies, such as the state's creation of the residential school system. The disruption of Aboriginal families and communities carried on long after the residential school system's closure, as the provincial government was given the legal authority to enact the child welfare policies on Aboriginal people, so long as they did not interfere with federal law. This history has resulted in a disproportionate number of Aboriginal children placed in foster care and, as a result, a number of Aboriginal people who access Site A and Site B were in foster care as children themselves. As one health care provider participant explained,

...[A] lot of our clients, they have no access to extended family or limited access to extended family, and so limited access to a role model. A lot of them have been brought up in foster care, or in and out of foster care... A lot of [the clients] have had previous involvement with the Ministry as children themselves, or as they've had children, a lot of them have had children removed.... (HCP8- Drug and alcohol counselor)

One of the woman participants explained how she was removed from her parents and the impact of being in foster care:

[My children] were taken away from me when my daughter was six and my son was three. They took them away and I fought for three years. Well, the thing they did was they used my past against me because of the way I was brought up in foster homes and I was neglected and abused and so they used my past against me. (WP5) 
What also appears to be largely the result of this history of disruption of Aboriginal families and communities is that many Aboriginal women lack the family or community supports that a child protection social worker would consider reliable assistance for raising children. For example, of the nine women interviewed in Phase Two, only two were living with another adult at the time of the interview, and of those women only one was living with the father of the children. Several health care providers interviewed in Phase Two emphasized that being a single parent or lacking family and community supports is a common challenge for women involved in the child protection system. As one health care provider said,

.... [T] here's a lot of single parents, [women] who identify as being single. They may have a boyfriend and/or partner but [they] cannot rely on them or depend on them so they identify as being single. (HCP 7-Family Aboriginal outreach worker)

The challenge of not having someone to rely on was consistently relayed in both the women's and health care provider's interviews in Phase Two. One woman described what her social worker told her she must do to regain custody of her children, which included relocating to a shelter and leaving her partner.

I lived [in the shelter] for six months but I still wasn't, you know, didn't get the proper help to be a proper parent. I didn't, you know, I was still unsure of everything in this world. Then they wanted me to leave the father and I was like what, he's the only guy I know here in town, I don't have family, like what the hell are you guys trying to do? (WP4)

The assumption that everyone has a family or supportive partner is contrary to the situation for many women who access Site A or Site B, as one health care provider explained: 
And we think we all have family, and that's such a judgment call, not everyone has family. And even if you do have family is it a safe family in the Ministry's eyes? If you brought your child to your mother, if she has a long extensive history with the Ministry will you get yourself in more trouble? So you're forced to lie or just do nothing. (HCP7Family Aboriginal outreach worker)

One challenge of raising children alone or with limited support is that many of the women do not have safe options for child care. One woman shared a story of paying a friend one hundred dollars to babysit her son while she went out for an evening. The friend took her hundred dollars, left the child alone, and called MCFD to report that a child had been left alone in an apartment. This was a person in whom the mother had confidence, as she had looked after the child several times before. The outcome of this event was that the child was removed without visitation privileges for a month. Similarly, a health care provider recalled helping a mother who was in great need of support:

When I worked at [another clinic] I had a young, teen mom who came down with the flu. She had no family here whatsoever, she phoned me hysterically crying, "[Participant's name] I want to throw my child out the window. I just want to sleep." I knew she had the flu, I said get him ready, I'm coming to get him. I went to her house, picked up her child and I said I'm going to keep him for five hours and I want you to go to bed. Go in your house and go to sleep, call me in five hours. So I took him to my work and my boss was a little bit upset with me and I said, you know what, this child is not going to get hurt. Like yes, I'm way out of line and, yes, I shouldn't be doing this but, yes, we're saving a lot of other things that could happen. Five hours later she showed up at my office, she had slept, she had eaten, she looked completely refreshed and she thanked me. She was 
like “I don't know what I would have done; I probably would have hurt him because I was so mentally exhausted.” (HCP 7- Family Aboriginal outreach worker) This story sheds light on how trying it is for a mother to parent alone. Not having supportive people in one's life is a significant barrier to successfully parenting children, particularly if the mother is under investigation by a child protection social worker.

\section{Structural Inequities and the Context of Poverty}

All of the women were unemployed at the time of their interview and therefore relying on social assistance or other means for financial support. All health care provider participants emphasized that poverty is a major issue for most of their pregnant and parenting women clients. However, poverty cannot be discussed without considering how Canada's past and present colonial policies have been key factors influencing the socio-economic status of many Aboriginal people. As one health care provider stated,

I realized that if I wanted to do something to prevent the apprehensions of children... it is because of the colonial policy culminating in the residential school experience. It has been over a hundred years, the disruption of the culture, and hence the loss of identity. So people come through our doors, most of them don't know who they are, where they belong, and poverty. Poverty is held against them and it is straightforward, you can deduct that straight as a result of the colonial policies that Canada has held (HCP2Registered Nurse).

The disproportionately higher rate of poverty among Aboriginal people versus the dominant Euro-Canadian populations must be understood as the result of colonial government policies. These historical policies continue to influence the socio-economic circumstances of many Aboriginal peoples. 
One of the women related how poverty, in particular, impacts how she sees people being treated in healthcare:

I believe it's because of poverty that we get judged for the situations we are put in and facing. It's because of the poverty they judge you, because you're on welfare, they judge you because you can't afford a pair of new shoes. They judge you because you look a little rough, you know, like they don't know some of the circumstances people are going through, but they judge them. (WP4)

Living in poverty or below the poverty line often in extreme poverty, directly impacts a person's ability to parent. One of the main challenges associated with poverty is that of attaining safe and adequate housing. From the health care provider participants' perspectives, women living in poverty often live in environments that are not viewed by the child protection system as safe or adequate for raising children. As stated in the literature review, attaining safe, affordable and adequate housing is highly problematic for parenting women living in poverty. One of the participants explained, "[These women] are living below the poverty line. They live in homes that are pretty run down and pretty inefficient for how many children and how many adults live under [one] roof." (HCP7- Family Aboriginal outreach worker). In this next excerpt, the woman explained how inadequate housing prevented her from being able to get all of her children back in her care:

I've got six children, one more still isn't [in my care] until I get a bigger place because like right now I'm in a one bedroom with five kids. And my two older girls just got returned...I asked the manager downstairs and she said it was okay and so I recently got my older kids back so there's like five of us in here right now. (WP6) 
Not having affordable and adequate housing prevented this woman from parenting all of her children; her apartment did not meet the required size for housing seven people.

In this next excerpt one of the health care providers expressed that for many mothers

living in poverty, their daily concern is survival, and therefore women are often not able to attend to their own health issues:

... [A] lot of [the women] are on social assistance and they live in the shelters and it's very hard for them to kind of deal with anything because they're just dealing with daily survival. You know it's like food, shelter, maybe dealing with partners who are abusive and so what I try and do is work with them so we do harm reduction.... (HCP3-Drug and alcohol counselor)

From the health care provider participants' perspectives, the social determinants of health (food, income, and housing) must first be addressed before most pregnant and parenting women decide to receive medical services. The previous quotation also highlights the issue of violence and abuse, which is discussed next.

\section{The Context of Violence and Abuse}

The majority of the women participants from Phase Two told stories of experiencing violence and abuse that included physical, sexual and emotional abuse; as well as other forms of violence. For example, one participant had a child who was the result of a rape and another described a story about almost not surviving a partner's violence. The health care provider participants also emphasized that many women involved in the child protection system have suffered physical or sexual abuse.

In the previous chapter, I discussed how violence, particularly intimate partner violence, is often a barrier to parenting because the father or partner is seen by a child protection social 
worker as a threat to the child. One woman participant reported that MCFD tried to apprehend her child at birth because her partner was known to be abusive: "... [A]nd that's what ["The Ministry"] tried to do with my first son, they tried to walk into the hospital and apprehend him right from the hospital because I was living in an abusive relationship." (WP4). The challenge for this mother was that this abusive partner was the only person in her life. She explained how "traumatizing" it was for her to leave her partner because she had no other family or friends, and that she would not have been able to parent her child without the support she received from the staff at the Site she attends.

The findings from this phase reaffirm the findings from the first phase of the research, which indicate that leaving a violent or abusive partner can be extremely difficult for a woman who has no other supports. In this next excerpt the health care participant explained how violence is dealt with by child protection social workers:

So with violence, I mean there is a type of restraining order that can be obtained as well within the child protection system, but the way the child protection system will usually deal with it is they put the onus on the mom, right. But they have, within the court order, a supervision order with conditions as to what can happen. So, the dad cannot be around the home, the mom must ensure that he's not in the home. The mom must call this person if he does come around, and things like that. So they can, yeah, [the child protection system] can put like similar conditions where the partner can't be around. (HCP 5- Child protection social worker)

The form of violence most commonly discussed by the women participants was intimate partner violence. A number of the women had experienced several violent or abusive intimate 
relationships. The woman participants seemed to find counseling or having a supportive person that they could talk to about it to be beneficial, as this woman said:

Well when I needed somebody to talk to, I could just open up to anybody [at the centre] that I felt comfortable with, and I just let everything out and everything [I say] is confidential. So that makes a big difference, like there's only me, I've been hurt all my life and I've been in abusive relationships. (WP8)

Leaving a violent or abusive partner is challenging for women with no other supports, and yet leaving a partner can be mandated by a child protection social worker. Helpful healthcare services for women who have experienced violence or abuse include: creating a safety plan for women currently in a violent or abusive relationship (as discussed in the previous chapter); being non-judgmental to women who stay with an abusive or violent partner; and having supportive services such as counseling to allow women to talk openly about their life circumstances.

The findings from Phase One and Two indicate that from the participants' experiences, the pregnant and parenting women involved in the child protection system face numerous sociopolitical and economic disadvantages. This is similar to what the literature indicates: that a disproportionate number of Aboriginal children are in foster care, and that mothers at risk of having their child apprehended often live alone or with limited supports, live in poverty, and have experienced violence and abuse. Understanding the common socio-political and historical context of many Aboriginal women involved in the child protection system helps one to understand how having a child protection investigation or losing custody of a child can further complicate a woman's life. 


\section{The Bureaucratic Structures Governing Child Protection Agencies}

The following section focuses on the bureaucratic structure of the child protection system. In this section I present the findings related to the common challenges the participants experienced when dealing with the system. These challenges fell into three categories: a perceived lack of consistency from one child protection worker to another; frustrations with the amount of power within the system; and the limited supports available within the child protection system.

\section{A Lack of Consistency}

The majority of health care provider and women participants voiced frustration with their experiences of child protection social workers being unclear as to what women or families were required to do in order to regain custody of their children. Several health care providers talked about there being great variation from worker to worker as to what mothers or families must do to either maintain or regain custody of their children, as this participant stated: “...[A]nd there's no consistency in what "The Ministry" does, like from worker to worker. Some workers will be okay but others are so hard-lined..." (HCP3- Drug and alcohol counselor). This perception of lack of consistency is echoed in the following excerpt:

One social worker may say, okay, it's okay if they're using this [drug] and set up a safety plan around how this parent may use [drugs]. Whereas another social worker will come in [and say] no, you can't even smoke pot; it's a hundred percent abstinence, you can't use anything or we'll remove. And it also has to do a lot with that individual's history and what they've been put through. (HCP 7- Family Aboriginal outreach worker) As both excerpts highlight, lack of consistent guidelines can be a challenge for health care providers trying to support and advocate for women involved with the child protection system. 
Some of the health care providers expressed that it is difficult to support women when it is unclear what the outstanding issue is preventing a woman from getting her child or children back.

Additionally, some women participants expressed frustration with what they felt to be unreasonable expectations from their child protection social worker. One woman described the contrast between her previous and current social workers:

[This social worker] is helpful, and not like the one [from a different agency]. She just like made me do everything over and over, like so many parent programs. And then she still wasn't happy with that and then I was like trying to get as much visits as I could but she only gave me one per week. And I wanted to see my kids more and she said, well maybe we could get you to see [son's name] but no he's not allowed right now, and the kids are going to be in school anyway. And so I said, can't I like have them after school... and she just always said no. (WP6)

This participant continued to talk about her frustration at how she felt her previous child protection social worker seemed to want to prevent her from parenting her children rather than trying to support her to be successful in caring for them. Similarly, another woman participant described how she started to become critical of what was being asked of her by her child protection social worker:

I took my son [with me] to treatment; I stayed in treatment for a month. [“The Ministry"] tried to tell me oh when you come back from treatment we're taking your kid. I said no you're not, now you're starting to set off alarms. I said you know what, I'm taking my son with me to treatment to better the life for me and him. And if you guys are going to step in and take him, him and I have bonded...you want to take him away again? I said 
that shit's not going to fly. And so I fought them for a whole month. ["The Ministry"] said, okay, we'll let you keep your son if you go back to the shelter and find a good home for you and your son. So within two weeks of coming out of treatment and going everyday with him in the stroller I found a place. And I got out of there and then they said, well, you weren't allowed to leave. I said, hey, that's not right, I said I did everything you asked me to do, my drug tests everything has been clean. I'm in a program, I've got furniture for my place, I've got all this stuff set up, too bad I moved in so what are you going to do about it now? I said you guys told me in mediation ${ }^{8}$ I can do this and if I was strong enough to do it, I proved you wrong, I said I did it all. I moved in my place, I paid the rent, come see my house. (WP4)

This mother voiced several examples of how she believed she was doing everything that had been asked of her in the mediation meetings and by her child protection social worker but because she did not, for example, take a specific counseling program, the result was another conflict with her social worker around her ability to parent. The majority of participants said they were trying to adhere to the expectations of their child protection social worker; however, it was difficult to meet their child protection workers expectations given the circumstances of their lives and the approach of the child protection social worker. This next quotation from a health care provider participant conveys what she thought would be helpful for mothers who have a child protection social worker:

[What would be helpful is if] things were set up and all the checks [were] in place prior to [the woman] delivering a baby, that will help alleviate stress. I think when the Ministry

\footnotetext{
${ }^{8}$ Mediation or integrated case management meetings occurs after children have been removed from a parent or guardian. It is intended to provide the parents or guardians with a voice, and the mediation meeting is facilitated by an impartial, trained mediator. At these meetings there is a lawyer for the mother or parent and a lawyer for the Ministry. Ideally the mediation session happens in a non-intimidating location and the parent is able to bring advocates for support. The goal of mediation is to find a mutually agreed upon best plan that will maintain the child's safety (British Columbia's Ministry of Attorney General, 2011).
} 
is more transparent of what they expect and what they want, and very clear and direct, which I don't find them very clear or direct. [The Ministry workers] don't want to be the bad guy telling the mom what she has to do. They wait until it's too late; well you should have done this, [and the women say] well why didn't you tell me? If you had told me I would have done that. So I just think honesty, transparency and, acknowledging the parents' past but not getting the parents stuck there. (HCP7-Family Aboriginal outreach worker)

As this quotation summarizes, participants found the child protection system challenging to navigate when they experienced child protection workers as being unclear or inconsistent in their expectations. Most participants seemed to think that upfront and clear expectations from child protection social workers would be helpful. As discussed below, the participants also saw the power that "The Ministry" possesses a real challenge for parents involved in the child protection system.

\section{Power of the Child Welfare System}

The power of the child protection system was brought up in a number of the participant interviews. These participants believed the child protection workers had substantially more power than the mothers when it came to child custody and visitation decisions, as this participant explained:

...God knows that I don't think that the people who work [for the Child Protection system] are bad people because I don't. I just believe that they work for an institution that has a lot of power. And the colonial policies and the poverty, inter-generationally has affected the [Aboriginal people], so do things differently - meaning more support and less apprehensions. (HCP2- Registered Nurse) 
A number of participants thought the power that child protection social workers have constantly "loomed" over them. The legislative authority within The Child, Family and Community Services Act allows certain child protection social workers to have a very high level of authority. This is necessary to a degree because the child protection workers are focused on the best interest of the children. From the perspective of the study participants, however, the significant power differential between the parent/guardian and MCFD causes them to feel completely powerless. The women may be required to complete a series of parenting programs and attend a number of meetings, but complying fully did not always result in the return of their children. The end result is that women feel defeated, which can lead to "backtracking" (e.g., using drugs or alcohol). In the following example, the participant expressed her frustration and feelings of powerlessness in relation to her child protection social worker's decision-making. This woman and the father of her older children had a no-contact order - a court order prohibiting them from having contact with each other. Although she did not purposefully plan to meet the father, someone saw her speaking to him and told her social worker. The participant believed that this incident prolonged her children's time out of her care. She explained, [The father and I] just quickly said hi and I was like I've got to go and I was like just rushing to the grocery store and then rushing to [the child protection worker's] office. And I was so mad, it just sucks that they have the upper hand on having the kids in care for such a long period of time, for such a stupid small thing. It's not like I was with him, or if I was using or something, because I don't do drugs... but saying hi to the father for like a minute or two, just telling him how the kids are doing, I thought that was like, just bullshit... (WP6) 
Similarly, a participant shared her frustration with the way she was treated by "The Ministry" child protection workers and her feeling of insignificance:

I just think that [“The Ministry"] is totally unfair to society, to people, to human beings who should be treated fairly. I don't think the Ministry is doing a very good job of informing people of what needs to be done in order to have their child put back...they just hit you with court papers, court papers, court papers. You do this, do this, do this; well what about me? Where's about my belief, my culture? You know, like who do I go to? Where do I go? They, they don't give you any information on where to get help. It's just this is where you go for drug testing, this is where you've got to go, you know, this is where you've got to be. Well what about me, what about my feelings? (WP4)

The women participants felt as though the expectations on them were very high and at times unreasonable. Most of the women were trying to parent with very little support and therefore what they wanted from their child protection social worker was assistance and support with parenting. Participants also expressed concern about the limited supportive services available within the child welfare system.

\section{Limited Supportive Programs and Services within the Child Protection System}

The limited supportive programs and services within the child protection system were expressed as problematic in both the women as well as the health care provider interviews. Many of the participants believed that if more holistic supportive parenting services were available for parents it would prevent some of the child apprehensions. As this health care provider stated:

What I see is an underfunded Ministry who seems to go one or two ways; they either ignore the problem until it's a crisis or they jump into it before anything had happened and their automatic response is to remove and then work on the issue. There's not a lot 
of in-house teaching and learning and parenting that is done with, with children still in custody of their parents. And I think that would be a really good place to start because there's sort of that whole spectrum of reasons for Ministry involvement and at the worst end of the spectrum absolutely maybe, maybe children should be removed for their safety. But I'd say the vast majority, if they were given some parenting skills, which a lot of them haven't been shown and haven't been taught, they could be really good parents.

\section{(HCP4- Physician)}

This health care provider believed that more comprehensive parenting programs could reduce the need for apprehensions. Along with parenting programs, the health care providers suggested that assistance with affordable childcare and safe housing would be extremely helpful for women. One health care provider explained:

I'm sure any of the staff here would say that's something that's a limitation of the system right? They should be providing better childcare options for moms, safe child care options right so that they can take breaks, so that they have a safe place to go. I mean we've also talked about the need for places where moms and babies can go together, that's not specifically transition housing for women fleeing violence, which again they need more of those too. But even if that's not the case like we have lots of women with babies or children that do need a break. They're not maybe in that moment fleeing violence from their partner right but they need a safe place to go and a break and they don't want to be apart from their child right? Well to have a supported transition home right where they can just stay for a little while until they get back on their feet again or feeling comfortable in going back out to the community right? I mean that's something that is much needed and would be very helpful. (HCP5- Child protection social worker) 
The idea of having supportive housing with staff employed 24/7 available for women when they need help with childcare was seen as very effective in preventing children from being apprehended. Since women involved in child protection investigations were often raising children alone or with very limited support, this type of housing could be very beneficial.

A number of the women and health care provider participants said that when mothers or parents ask for support from their child protection social worker, the workers are often not available. In this next example, the woman participant explained what she appreciates about her new child protection social worker:

...So you have to jump through a lot of hoops [with MCFD] and I've been fighting my addiction for 30 years, since I was thirteen. So I was pregnant last year and I relapsed because I found out I was pregnant with twins. And I told my social worker I was going to go and use and that [my kids] needed to be taken.... But now with the new social worker it seems a lot better. She's more caring, this other social worker, it's like she just threw her hands up and that was it. (WP8)

Similarly, a number of women participants provided examples of friends or family members asking MCFD for supportive services but not getting any help. The absence of supportive services was said to result in "crisis moments," where a child protection social worker would have to remove a child. The women participants appreciated having a child protection social worker they felt was "on their side." One woman even moved to a different city because she was told that if she got into a particular agency there she would be linked up with a more supportive child protection social worker. She explained,

... [My old social worker] she just like had no, I don't know, she doesn't know how Natives are. Like I don't know my family we're like really close and we help one another 
out if we need help. And like my grannie had seven kids, my mom had six kids and I have six kids, so it's like we all like have big families and we all help one another if we need help. Then [my old social worker] because [my daughter] acted pretty mature back then, well she still is now, she is so mature, [my old social worker] felt that she had to take care of my other kids when I left the home and that's why she was so mature [because she needed to take care of the other kids].... And she was just always criticizing what's going on and whatever. I just had like a really rough time in [that city] so that's why I moved to [here]. (WP6).

In the community where Site A is located is a women's transition house to which mothers are sometimes sent if the regional child protection agencies are not confident that a woman is ready to go straight home with her newborn. The challenge is that this shelter is quite regimented with programs throughout the day. The staff at Site A have negotiated with the transition house, explaining that the women may need some leniency in terms of attending all of the programs when they first move in. They use this transition house quite often if the women agree to it, and it appears to be helpful in preventing an immediate apprehension after birth. This is one of many community resources with which Site A has connected in an effort to support the pregnant or parenting women. However, participants at both of the Sites expressed a need for more supportive and preventive services for pregnant and parenting women dealing with complex life circumstances such as poverty, violence, parenting alone, or substance use.

\section{Aboriginal Women's Experiences with The Child Protection System and Process}

This section examines the findings related to Aboriginal women's experiences with the child protection system and process. I begin with an explanation of the terms commonly used in the participants' interviews and then discuss a number of the participants' stories of having their 
children removed. Some of the stories are told by health care providers, as many of them have supported women professionally and personally through the experience of having their children removed. The removals were commonly framed by both the health care providers and women participants as highly traumatic. This section concludes with a description of what the health care providers from Site A and Site B recommend to women who are likely to be involved with the child protection system.

\section{Common terms used within the Child Protection System}

I begin this section with an explanation of the common terms used by the participants when referring to experiences with the child protection system (often referred to by participants as "The Ministry"). One of the terms often mentioned in the participants' stories refers to having an "alert" out on a woman. In the following excerpt a health care provider explained what happens when an "alert" is put out on a woman,

So, for instance, you're pregnant and you go to get your welfare check and someone sees that you're pregnant [and] they call the Ministry. Anybody in the community, if your landlord sees that you're pregnant and knows that you're using, so a hotel owner, they can phone the Ministry and an alert is put on your file. Because the Ministry can't really intervene until the baby is born. So like that happens for many of our clients, it could be a family member, it could be anybody. It could be a police officer who picks them up and sees that they're pregnant. [That person] phones the Ministry and an alert is put on their file. So when they go to the hospital it's big alerts, and some of them are province-wide; some of them can be across the province. So that when they show up at the hospital their names goes in and a big red flag comes up and the Ministry has to be called. (HCP8Drug and alcohol counselor) 
A number of the women participants told stories of landlords, police or people within their apartment building calling "The Ministry" either before their child was born or after to report a concern about the safety of a child or children. A number of participants thought the women's pasts continued to follow them no matter how well they were doing at the time of their pregnancy, as this participant explained:

And their past keeps getting brought up which is very wearing on them when they feel like they need to give up, you know, what they're never going to see me as being a good parent. They're never going to see that I've stopped doing that and I've started doing this. And we just keep encouraging, just don't let them beat you down, you can do this, it's just another challenge you have an opportunity to prove to them. They're really trying to push your buttons, you have an opportunity to prove to them, no, you can keep pushing that button and I'm not going to use. (HCP7-Family Aboriginal outreach worker)

The participants generally agreed that once a woman becomes known to "The Ministry" there is Ongoing "Surveillance" of the Woman. One woman explained how stressful it was for her while she was pregnant because she felt she needed to prove that she could successfully parent the child she was carrying:

They were filing paper work to get him taken away when I was pregnant when I was like twenty-four weeks, like I, like when I spoke with my old social worker in [another city], she was saying "IF we don't take him away" like just letting me know she's going to take him away... [S]o, I don't know I was like really emotional and that's when I applied here, when I was pregnant with him [pointing to her ten month old child]...It was so 
stressful when I was pregnant like all I did was worry about if they were going to take him away... (WP6)

The duty to report any concern about the safety, health or well-being of a child (or potential child) is vital without question, as child safety must always be a main concern; however, a number of participants struggled because, unfortunately, some people call "The Ministry" for unrelated reasons or because they are in conflict with the mother. The outcome of those calls can be devastating, as this participant explained:

It's hard because if the Ministry, like they will investigate calls that people make and they'll call people in and say we heard that you were using and you're partying, and the woman may not have done that. And a lot of the times when calls are made they're investigated, and sometimes the kids are taken away because of those calls...And whether [the calls] are legitimate or not, like whether this person is just calling because the want to get back at this person from something totally different (HCP3-Drug and alcohol counselor).

This health care provider explained that some women will actually try to hide from "The Ministry" in an effort to keep their child if they know there is an "alert" out on them or if they have been "red flagged" to "The Ministry", as she explained:

Okay we're dealing with a few people who are pregnant and one who has just recently had a baby in [name of city]. And one of the things that [the women] do because they know that they're going to be red flagged because they have used cocaine or whatever their substance is, they will try everything including moving away. Like one of our young women who is pregnant she's moved into [a remote area] because she knows that she'll get help from the band, and she thinks that the band will help prevent the Ministry from 
apprehending her baby when her baby is born. And the result of this is that she's coinfected, she has HIV and Hepatitis C. And so it's very difficult for her to get some of the services, oh she's on methadone (HCP3- Drug and alcohol counselor).

This excerpt highlights one of the consequences of women trying to flee apprehensions, as the mother in this example decided to move far away from the healthcare services that she needs such as a physician that is licensed to proscribe methadone. This women moved to an area where methadone programs are not available therefore she will longer have access to methadone, an opioid commonly used to treat moderate to severe pain or to treat heroin use. A major challenge for some Aboriginal mothers and families is that their own history with "The Ministry" has been very negative. Furthermore, a number of the participants thought Aboriginal women continue to be particularly over scrutinized when it comes to parenting practices, as this health care provider explained:

And lots of Aboriginal children as you know are apprehended and a lot of them for no good reason. And so [the women] got a real cause for being concerned, which is part of our role here is to, you know, hopefully try and play a small part in changing that kind of thinking... But [when] you learn more about what's happened to Aboriginal people [at this place] and then you get an understanding of why things are the way they are and it certainly makes you hopefully become more compassionate and understanding [towards Aboriginal people] and their plight. (HCP1-Registered Nurse)

Therefore one of the challenges in regards to everyone's duty to report suspected child abuse or neglect, under The Child, Family, and Community Services Act, is that some women thought they were unfairly targeted, particularly if they had previous involvement with the child protection system. The health care provider participants from Site A and Site B have a recommendation for 
such women, which will be discussed later in the findings. However, as this health care provider explained, even when a child is apprehended and then given back to the mother, it is hard to recover from the initial apprehension:

You know ["The Ministry"] actually do want to be more supportive during the pregnancy, whereas in the past they obviously wouldn't get involved in the mother's life until the baby was born, and then they would show up at labor/delivery, they would apprehend the baby and then they would do all their investigations. And then sometimes they would find out, okay, this isn't actually too bad and the baby can be returned. But obviously a lot of damage has been done by that time; the bond has been broken and the mother even if she had been struggling with substance use during her pregnancy if you take her baby away she's, her chances of relapse are pretty high because she's broken hearted, they've taken her baby away... (HCP1-Registered Nurse)

\section{Traumatic Experiences with the Child Protection System}

A number of women relayed traumatic experiences with the child protection system. For some of the women and health care provider participants it was quite difficult to talk about these memories; however, when I offered the participant the option of stopping the interview or taking a break, each participant insisted on continuing the interview and gave as their reason for moving forward that they wanted their story to be heard. Therefore, I have included selected stories here, as a number of women stated that their reason for participating in this study was to share these stories so that people working with families involved in the child protection system could learn from them. In this first story the mother relayed her experience of having her child taken from her at birth: 
They made the doctors do a drug test to see if I was using while I was pregnant and the test came back clear and they still wanted to take my child away from me. So my son was only ten hours old and they took us and they moved us into this shelter, which was so traumatizing and I never been in a shelter in my life. And I thought god this is the worst torture I've ever experienced. We had to do drug testing and it was just bizarre how I was treated at first. (WP4)

This woman was asked to go to a transitional housing shelter with her child because there was concern about the safety of her living situation. She was told she must go to a shelter because she could not parent her child if she was living with her partner, who was known to be violent and abusive. However, she did not know she would be asked to leave her home and her partner. She thought she would be able to go home with her child because she remained off drugs and alcohol once she learned she was pregnant. What stood out when I interviewed this woman was how extremely emotional she became when she talked about the "trauma" and "devastation" of having her children removed from her care.

In this next story the participant talks about her child who died in foster care. Her daughter was abused in foster care and subsequently died in the hospital. In this quotation she talks about a memory of being pregnant with her daughter:

And I liked the way I was pregnant, I sang to her when she was in my belly and stuff like that. I'll never forget all the memories I have. But it's quite hard because I got the other memories of the last time I saw her and the lies that were told me. The Ministry told me that she was sick with the flu, and her brain wasn't even working and she had tubes in everywhere and bruises from head-to-toe (WP1). 
This participant talked about wanting to use drugs and alcohol after this experience but she was able to stay off of them, with the support of the staff at the centre she accesses. She emphasized that she knew she had to stay "clean" so that her other children did not end up in foster care, and at the time of the interview she had maintained custody of her other children. She attributed her success to the support she received from the staff at one of the Sites, which included setting her up with a lawyer, helping with transportation and ongoing drug and alcohol counseling. This was not the only story of abuse or neglect within the foster care system, as this participant explained:

I recently got my son back, he was in care for like nine months; I got him back last year. But my son got hurt in care so that affected me big time. The removal of my son and being a single parent was totally devastating to me... The fact that they don't take into consideration that when they remove your child that you go under a lot of stress... (WP4) The women and health care providers interview data suggests that there are trauma issues related to no longer having custody of a child as well as to the process by which the removal is carried out. One health care provider shared her concern about there being a lack cultural sensitivity at the time of the removal:

...I think it is traumatic and I think particularly with how it's done where, you know, they just sweep in and I don't think there's a lot of cultural sensitivity, and I don't think there's a lot of looking at, oh well you know, in this community that's a reasonable thing to do, to have a bunch of people living in the house or you know, to leave the children in the care of this person or that person because that's the normal behavior. But because it's not the way, the narrow box that the Ministry sets forward it's not appropriate, and the Ministry is the one with the power. (HCP4- Physician) 
The removal process seems to vary with the child protection agency or even the child protection social worker. At Site A, the staff has been pivotal in improving the procedure of child apprehension at the local hospital. One participant described how the procedure has evolved and improved over time:

So one of the very first things that we did is when I started this work is that we had a community of public health nurses, NICU, nurses at maternity ward, clinical instructors, social workers that said, yeah, there has to be something different done about the apprehensions and how it works... It's awful the way it went, ["The Ministry"] could just come up there and say you don't get to go home with a baby, no congratulations how are you? Or what a beautiful baby... We invited MCFD [and said] these are all our concerns and MCFD explained a little bit how they worked and what their mandate was and how they have to do things because they' re on a very stringent sort of guidelines and tickety box. And so that helped us understand better that they're under the gun too. But we said well... we should actually have a room where people can sit down and we should also work together. So there was policies that you couldn't just come in unexpected and just [go into] a NICU and say well this baby is not going home with you, they would have to have a place where they could sit down and talk to people and that's bad enough. So with our patients we always try to be with the patients and support if [the child] goes to MCFD so it's very rare that they're on their own unless they really want to... (HCP2-Registered Nurse)

The previous excerpt indicates that within the community the child protection agencies seem to be trying to work more collaboratively, which ideally will help reduce some of the trauma that occurs with child apprehension. 
Another outcome of having a negative experience with the child protection system seems to be that many women live in constant fear of having "The Ministry" involved in their lives. As this health care provider explained,

[The pregnant women or parents] who have addictions really have a tough time, because they're, they're under constant threat of the Ministry, and if they're involved with the Ministry, it's really hard for them to, to deal with the Ministry because they're first of all like they're watched so closely. They have to come in for urine drug screens. I was dealing with someone yesterday whose children, she had a slip and the Ministry found out and oh she was in detox that's how they found out. And then the next thing she knows she was going home to be with her children and her kids were apprehended (HCP3- Drug and alcohol counselor)

Living in fear of having a child protection social worker become involved in a women's life seems to be a common challenge for women who have experienced child protection investigations, and this is echoed in the following quotation:

I can honestly say that I haven't met a client who wants to hurt her child and [they will] hopefully make the right choices if they're offered to them. But the fear of apprehension is huge and if the client has a relationship with somebody that she can trust and can reach out for them, they don't want to hurt their kids... (HCP8- Drug and alcohol counselor)

The women participants talked about the constant stress they felt having to follow all of the expectations that were required of them to maintain or regain custody of their children, while the health care providers highlighted the impact of the child investigations on women and families: And they have the opportunity to really, to really develop the family. And I think people who have had their children removed it just, I see them every day and the hurt and the 
heartache and the negative outcomes from people who have had their children removed from their care or been removed from the care of their parents is something we see every day at [name of city]. The depression, the anxiety, the inability to sort of move on with their lives, and with their jobs. (HCP4- Physician)

Several participants expressed their frustrations with the process of child protection investigations, particularly when the child is removed because the person who makes the report of suspected child abuse or neglect remains anonymous. The anonymity is required to maintain the safety of the reporter. However, the lack of information at times can be frustrating to the mother. Furthermore, some women expressed frustration with not knowing where their child was while in foster care (they may know the city but no more) or not being able to talk to their child or children while not in their care. In fact, some of the participants went as far as to compare the current state of Aboriginal children in foster care to what happened with the residential school system. As this participant said, Okay they don't have [Site B] to back them up. They need [Site B] all over the place seriously need it, instead of Healthiest Babies Possible they've got that in every, every small town all over the place. They need [Site B] to at least give these gals a fighting chance. And I look back at the residential school and what's the difference? Seriously what's the difference from [the Residential schools] to the Ministry now; there is no difference at all... because it all boils down to the Ministry coming in, court workers saying I don't believe you, let's take this child and pick her up. It's no different from way back when, that apology meant nothing because they're still doing it. (HCP5-Child protection social worker) 


\section{Early Referral to MCFD}

One of the main recommendations health care providers made is an early referral to MCFD, if it appears inevitable that the child protection system will be involved in the woman's life once the baby is born. The majority of health care provider participants from Site A and Site B spent a significant amount of time in the interviews explaining the early referral process and why it was developed. Its purpose is to present documentation of the progress that a woman may make throughout her pregnancy (e.g., clear urine tests, or attending prenatal visits). It is made clear to the women that an early referral is completely voluntary and that they do not have to agree to it. However, both Sites to have found it to be beneficial in preventing some child apprehensions that would otherwise occur at birth.

For example, if a woman finds out she is pregnant, chooses to go into detox and stays clean, the health care providers will keep a record of events such as clean urine specimens, prenatal visits and any parenting classes she attends. This way the woman's more damaging history (e.g. drug or alcohol abuse) may be less harmful when she goes into the hospital to deliver. From the health care providers' perspectives, if the woman does not connect with MCFD before the child is born and an "alert" is out on her, they have a more difficult time preventing the immediate removal and, once that removal has occurred, defending the women. In the following excerpt, a health care provider explained this process:

...And there's no legislation to cover this so what....we found was that in the early stages of [this program] five, ten years into it, we had a relationship with clients but the Ministry had none. And so when the client would show up at the hospital and give birth we know what was going on but the Ministry only had their file. And so they saw all of their past history, so anything that the client had done to change during the time they'd been with 
us was never really noted. So the children were still being apprehended. Our social workers worked with management to do what we call an early referral and it's only done if the woman wants it, it's completely voluntary. And what we encourage them to do is get to meet the social worker before they have the baby. If we know that the social worker is going to be involved, we say due to your past history, due to what's happened, why don't you just make the call, we'll sit with you, we'll sit in the first meeting and you can show them all the stuff you've done to change. You can show them that you have housing, you can show them you have this, and you can tell them and ask them what you need to do to be a parent, that's huge. So trying to get the clients to work with, with the Ministry in a more proactive way where they feel they have some choice has been really huge (HCP8- Drug and alcohol counselor)

A number of the health care provider participants from both Sites explained this process and that the process of assisting women to contact "The Ministry" prior to her giving birth is something that developed over time. Site A's relationship with the child protection agencies in its region has improved so much so that the child protection agencies will actually contact the staff at Site A if they have an Aboriginal woman who is under investigation with the child protection system and does not have a physician or other healthcare supports. Site A will then take on the woman if she agrees.

As stated, the early referral is completely voluntary. The health care providers emphasized that to suggest that a woman actually call "The Ministry" voluntarily requires significant trust within the health care provider-client relationship. Thus, gaining the woman's trust and building a positive relationship is essential but it takes time. Clients who access Site A and B often need to spend time with the health care providers to realize that they are there to help 
and support rather than to judge and discriminate, because for so many of the women their experiences within mainstream healthcare services have been filled with prejudice, racism and discrimination.

In the following section I present the findings that relate to healthcare access when child apprehension is either being threatened or actually carried out by child protection services.

\section{Healthcare Access in Context}

Understanding how the threat of child apprehension impacts Aboriginal women's access to health care is a complex issue. The impact of the context of the women participants' lives socio-economic status, historical and ongoing colonial policies that oppress Aboriginal women, violence, abuse, racism, discrimination, lack of family or community supports, previous experiences with the healthcare system, as well as child protection investigations - shaped the women's decisions or experiences with accessing healthcare services. The threat of child apprehension intersects with other socio-political and historical barriers that impact and shape access to healthcare and experiences within the healthcare system.

The majority of the women participants said emphatically that if their child needed healthcare they would never hesitate to take their child into a hospital or healthcare setting. Most women relayed that they would first go to the Site they regularly accessed but if their child needed urgent care they would not hesitate to go to a hospital. However, what was evident in all of the women's stories, and this was also relayed in the health care providers' interviews, is that within mainstream healthcare agencies (i.e., hospitals) there continues to be racism, discrimination and prejudice, which impact both the women's experiences in healthcare environments and their decisions to access services for themselves. 


\section{Ongoing Racism and Discrimination in the Healthcare Setting}

All of the women and health care provider participants provided examples of negative experiences within the healthcare system. These stories were mostly of racist or discriminatory health care providers. In this first example the participant explained how harshly she was told to leave the hospital:

... [F]or instance, like at [hospital name] I had pneumonia and I was really, really sick. And I was in isolation for about a week and they discharged me I wasn't even better yet. My pneumonia hadn't even [gone away] and it was during the wintertime. And the doc, one of the nurses, yeah, one of the nurses came in and said that your doctor is discharging you. I said I'm not even better yet and they said well it's time for you to go now. You need to get your stuff and you need to go, don't let me call security and sure enough she called security. Security literally came in, grabbed me behind my arms, dragged me down the hallway and threw me out the door, with pneumonia, in wintertime. And I went back in I said can I at least get a bus pass, a bus ticket? And they said this is not a charity this is a hospital. And right now I'm almost in tears . . . (WP9)

This participant was still very sick and spoke about how upsetting the experience was for her and how difficult it was for her to talk about other similar experiences that her friends have had in the hospital environment. In the following excerpt the health care provider talked about the racism and discrimination that she also has seen within the healthcare system:

But even being in hospital is quite intimidating and even, as a nurse, I worked for thirteen years in labor/delivery and I worked in the NICU and stuff like that as well. And even these places are not always the friendliest to our clients. And I think [clinic staff] being present there and visible, I worked [at the hospital] so I know the staff and, hopefully it 
makes a small difference. I certainly know the social workers there and we have a good relationship with them and so if you can go and be an advocate for the patients while they're in hospital. And it's a sad thing to say but, quite often nurses are, they can be quite racist or judgmental and as you probably know. (HCP1-Registered Nurse)

All of the women participants' targeted nurses as being particularly judgmental and racist. In the following quotation, a mother told a story about the nurses suggesting she had hurt her child when in fact it was a birth mark. The outcome of the nurse's judgment is significant.

[The mark is] on his neck, and [the nurse] comes in the room when my family came to visit and she's like oh yeah, we're concerned about his bruise or, his BIG bruise on his neck like do you know what happened, did he get hurt or something? And I was like did you not read the file? I said he had this at birth and I went to go bring him to the skin specialist to make sure that there was nothing wrong with him and they said it was just a birthmark and by the time he's two or something it will get smaller and just disappear. And she's [said] yeah we were all just concerned, and I'm like oh my god. So I called my social worker just to be on the safe side that she might say oh there's a bruise on his neck. So I told [my social worker] and she's like no, no, it's okay, I remember when you brought him to the skin specialist and I believe you. I know you're not that type of mother to hurt your child and I was just really pissed off. And I was like well I just want you to know because [the nurse] said that all the nurses were like really concerned. And I didn't want them to call you and then you get worried so I just called to let you know. And she's like oh thank you but, no, I think everything is okay... (WP6) This mother said that every time she took her son to the hospital the nurses were very unfriendly and quite racist towards her because she was "Native." She happened to have a child with a 
chronic illness and so has had several experiences with him needing to be hospitalized. The outcome of the overt racism and discrimination she experienced was that it deterred her from wanting to visit her child while he was in hospital. The majority of women participants believed that some health care providers in the hospital do not want to treat Aboriginal people. This is conveyed in the following two quotations:

Like me I'm not a judgmental person us Native women do get discriminated upon in the hospitals. But also too like and I have to say this because it depends on your attitude... the woman's attitude because I've seen women give the nurses or anybody else in there, doctors, a hard time and then they wonder why things happen right? Like it's not always the way but sometimes. Most of the time it's because the staff in the hospitals don't want to deal with us, which I think is sad... (WP8)

... [B]ecause I was so young and I was Native, oh yeah, I had a hard time in the hospital. This one nurse wouldn't even touch me, she wouldn't even put her hands on me or nothing and I didn't like that. You know because [this city] is so small and I was young and I guess she really judged me for [being pregnant]... (WP5)

The mother in this last excerpt spoke frequently about being very young when she had her first child, and how she felt she basically went from being a child to being a mother, because she had her first baby at thirteen. Being a young mother or having a substance use issue seems to particularly impact how some women are treated by health care providers, as this health care provider explained:

We hear from a lot of the girls that when they do end up in emergency with their baby, how they get treated especially if [a drug and alcohol program] is attached to them [then] they're seen as an addict or [having] a substance use problem and they're looked at very 
differently and treated differently, very undermined and not very well supported. So if their baby has to stay in the hospital it's more than likely the parent will not stay with them because they don't like the way they're treated and they may come and visit now and then... (HCP8- Drug and alcohol counselor)

Consequently, for a number of pregnant or parenting Aboriginal women, racism, judgment, and discrimination impact their decisions and experiences with healthcare. As stated, the majority of the participants agreed that if it came to the health and well-being of children, nothing (fear of child apprehension, racism, or discrimination) impacted their decision to seek help. However, racism, discrimination and/or the fear of child apprehension did have an impact on their decisions to visit children in the hospital or their decisions to access healthcare services for their own health concerns. One mother explained how she thought she could not leave while her child was in the hospital because if she did the nurses made her feel as though she was not being a good parent.

...[A]nd I was [at the hospital] for a whole week, a week straight like not leaving at all and then I asked to leave one day for just a day and that's when everything blew up, everyone started freaking out. And I'm like I see people going out all the time, you know, why, you know, I'm asking to for ... I need to go home and do some laundry and get some stuff together, I need to go pay some bills. And they're like why can't your boyfriend do that? And I'm like why can't I do that, you know? (WP7)

This participant thought she was being unfairly monitored in terms of how much time she spent in the hospital with her child so the next time her child was hospitalized she felt she could not visit. As she explained, 
Okay my daughter got sick again, she got the chicken pox a month after we got out of the hospital, and I didn't want to go; I did not want to be there. I in fact I feel bad even to this day for doing this to my daughter because she was two and a half months by then. I phone my mom and I said you have to come here because I'm not dealing with, if they put me on the same unit I'm not dealing with those nurses again... (WP7)

This is an example of how a negative experience in the hospital prevented the mother from being with her child on a subsequent admission. The outcome of not being with a hospitalized child is complex because it is often judged as neglectful by hospital staff and so can impact child protection investigations. Yet a number of mothers are not able to be with their child throughout their child's hospitalization for valid reasons, including other children at home, work, transportation issues, or fear of judgment.

Some of the women participants also expressed how previous bad experiences in certain health care agencies prevented them from going into the hospital at times when medical help was required. One participant had a health problem causing her pain at the time of the interview. When I asked if she was getting help for it, she explained why she did not want to go to the hospital:

I have to go for another ultrasound... because I'm in pain again... [but] I'm scared of hospitals... I just hate hospitals... I guess after my experience [at a hospital]. They lost my daughter's body [after she died in the hospital]. I was five and a half months into my pregnancy and they said they sent her to get cremated. Why would you send my daughter to get cremated, that's not up to you guys, that's MY daughter. I was going to bring it up but I just had to get myself grounded first... (WP8). 
This woman spoke about how she will never go back to that hospital because her daughter's remains were never found and she has a great deal of sadness at not knowing what happened to her daughter's body. She was especially upset about not having a place to visit to grieve her daughter. Some participants spoke more directly about how the fear of child apprehension impacts women's decisions or experiences accessing healthcare services. But again, it is the context of the women's lives and how that intersects with the fear of child apprehension that impacts women's decisions and experiences accessing healthcare services, as this participant explained:

I think for a lot of the teen parents their own history of being removed from their parents is huge. So when they're in front of any professional, they don't want to have the professional think they don't know how to parent or what to do. So they fumble and they don't ask questions, they don't ask for the support, they try to be everything and do everything without asking the questions or the support. And it's not a warm and friendly environment for teen parents to show up in hospital with a child and look like you don't know what to do. So they wait till the very last minute, they don't go, they may go to the walk in clinic instead at all hours of the night or just stay home. (HCP 7-Family Aboriginal outreach worker)

From this participant's perspective, being a teen parent, having been in foster care as a child, or the fear of looking like an inadequate mother can prevent mothers from asking for help or from asking questions. In the next example, a woman participant explained how the fear of having children removed impacted her decisions to take her daughter to the doctor:

Well it's like I said I couldn't, I was afraid to go to the doctor in case, you know, in case the Ministry was getting [the doctor] to write little notes, or how does [my child] look, 
you know. And I was so stressed out at the time that I did look like I was a mess. I looked like I was falling apart, my lawyer kept saying, you know what you've got to get strong, you've got to get strong in mediation you can't be sitting there crying and you can't be sitting there like you're falling apart. I said then what the hell, I can't help this. And every time I went there I was just shaking, I carried the shakes all the time and it was so nerve wracking and just scary and it was, it was a damaging thing and I was always falling apart...(WP4)

In this next example, a health care provider explained how sometimes women who have been able to stop, or significantly reduce, using substances during their pregnancy, but they will use drugs just prior to the birth out of fear of potentially having their child apprehended:

...So what they see and literature supports us in that way, is that we hope we get them early enough in the pregnancy soon enough and ready for all the paths of healing. And they quit all their drugs and alcohol, they do tremendous really, you know. But we often see that just when they are starting their labor they do cocaine and if they find that in their blood then and [health care providers] do the test and they find [drugs] in the baby even though they might stopped for the last six, seven months, but because of fear of apprehension [they use] (HCP2-Registered Nurse)

One health care provider said that when it comes to substance use and pregnancy, women are very truthful about their drug and alcohol use at the time of labor and delivery, because they want what is best for their baby. This health care provider's perspective was that the fear of child apprehension is always present for certain women but the fear is not only of losing their child, it is also a fear of being judged, and in this excerpt she provides a suggestion as to how health care providers can help reduce the feeling of judgment: 
As far as accessing care for their children I think the fear of having your child removed is there but also the fear of the judgments that they get. So if your, if your child falls down and has a goose egg on his head and you take him to the hospital they think that they're the only ones that are being questioned because of their background of poverty or their culture. And so being able to explain to them, you know when my child fell they asked me the same questions; being able to try and normalize some of that is helpful. But I think for this population the judgment has been so harsh for so long and the fear of having your children removed is huge. (HCP8- Drug and alcohol counselor)

The stigma women encounter particularly if they are known to use substances often stays with them throughout their life. The challenge for some women is that in order to access available services they must identify as a substance user. For example, at Site B the mandate is that women must have a past or previous issue with drug or alcohol use. The outcome is that when the mother or child goes into the hospital or schools they are often judged for being a substance user, whereas she may have exaggerated her substance use to access certain programs or services. Thus these women are labeled as 'substance users' and they face the stigma that substance users often experience, as this participant explained:

And unfortunately I think a lot of people say I have an issue with this drug and I have a challenge with this because they want their needs met and it might really not be an issue but because they are living in poverty they feel like they need to say these things in order to get our support. (HCP8- Drug and alcohol counselor)

For example, one participant described how when children go to school, teachers and other staff will see that all of the children's vaccinations are done at Site B, and labels the children as 
having drug-using parents. Racism and discrimination is not just an issue in healthcare but rather is an ongoing concern throughout the broader socio-political environment.

As discussed in the literature review, access to healthcare services for some Aboriginal women is shaped by a number of factors including the context of their lives (poverty, racism, single parent, violence, etc.), previous experiences with the healthcare system and the fear or having a child removed. The women's and health care providers' stories indicate that access to healthcare for this population must be improved. The health care providers and women provided several recommendations for improving the quality and the accessibility of healthcare services, which will be the focus of the last section of the findings.

\section{Setting up for Success While Navigating a Complex System}

This last section of the findings highlights recommendations made to improve the access and quality of healthcare for pregnant or parenting women with complex life circumstances. The recommendations included: an approach to care that is empowering and non-judgmental, which requires building a trusting relationship with clients, not forcing services on women and being flexible; education within the healthcare system about providing culturally competent care; providing family-centred care ${ }^{9}$ whenever possible; and increasing primary health care services to pregnant and parenting women with complex life circumstances.

\section{Empowering Women and Working Collaboratively with Women and Families}

All of the health care providers discussed how important it is to begin by creating a trusting relationship with the women clients, as many of the women they see have had their trust broken several times. The women participants spoke about a lack of trust in health care providers

\footnotetext{
9 Family-centred approach is meant here as an approach to care that includes the participation of all members of the family that the person identifies as family. This does not mean necessarily blood relatives but rather the people in the women's life that she identifies as her family.
} 
at the hospital setting; however, a number of the women participants emphasized how close they were to the health care providers at Site A and B. Building trust and creating a relationship with pregnant and parenting women struggling with complex life circumstances is the first step, as this participant explained:

...[I]t's amazing it's almost like just being present and being present over a certain period of time allows them to develop that trust right? Because I mean obviously this is a population who have had their trust breached over many generations and incredibly so, right by a system that is almost well basically faceless right? Because I mean you just have strange government officials come in and decide your children aren't going to be with you anymore and so on and so forth, destroying their communities...we're just always here right; You're always available and then over a period of time right and, you know, over a period of years of just always being here, they, you know, you develop a reputation of being, of having a relationship and being part of the community.... (HCP5-

\section{Child protection social worker)}

Thus building the relationship and gaining trust was seen by the health care providers as pivotal, and emphasized in this quotation:

Right but if they don't have the other stuff it really doesn't work because if you don't have the attitude to build the relationships you're not going to get the people in. They have to be able to trust that their needs are going to be met and that they're going to have choices. And if a woman comes in and we really think she needs healthcare and she's eight months pregnant and she hasn't had any healthcare and she says she doesn't want it, she doesn't get it, we don't force it on her. And that's one of the hugest things when 
talking to this population, the ability to have a choice of what they do with their bodies is huge. (HCP8- Drug and alcohol counselor)

Empowering pregnant or parenting women by giving them a choice and allowing them to have control over their own bodies is crucial. The health care providers all emphasized that pregnant and parenting women dealing with complex social and economic issues often require a substantial amount of supportive services. At Site A, many of the extended programs and services offered to the women have come through the health care providers' hard work in learning what services are available in the community that would benefit them.

The team within Site A that focuses on providing "wrap-around care" to pregnant and parenting women at risk of child removal spends a substantial amount of time with these women, as the participant explained:

So five percent of our population is pregnant moms, which is a relatively small five to ten at some given times, we give exponentially more time to them but I did that right from the start so, you know, given that like I said I was not a very good RN because a lot of other stuff didn't get done as well that had to be done. (HCP2-Registered Nurse)

Being non-judgmental includes not forcing women to take on programs or services before they are ready. For example, if women are not ready to seek prenatal care or go for a prenatal ultrasound it is not forced on them. This one of the reasons why Site B does not refer to its program as a "health centre," as this participant explained:

If you're pregnant and using [drugs or alcohol] the stigma around that is huge. And so in order for them to even access healthcare a lot of times we have to spend enormous amounts of energy doing outreach work, our nurses do that all the time. They may not accept healthcare, they may just accept the food bag so it will be knocking on the door 
handing them a food bag saying, you know, when you want to come in, come in, come in, it's up to you but we can give you more food when you come in. You can have a hot meal every day; it's trying to get them to a point where they feel safe enough to access healthcare. And for many of our street entrenched women that's the hugest piece and so a healthcare center we're not. (HCP8- Drug and alcohol counselor)

A number of the women participants had been clients of Site A and Site B for a significant period of time and many had been with the program or clinic for more than one pregnancy. The trend seemed to be that often the mothers would accept little medical care with their first child, but once they had built trust over time they were much more willing in subsequent pregnancies to access the medical services, as this woman explained:

My first pregnancy to be honest wasn't, I didn't really care about healthcare, I was seventeen years old, I was living on the street, I was an alcoholic and a drug addict. You know I wasn't ready to be a mother at all...I got some [healthcare] not much, I got some help from [name of program] ... and then I got pregnant [again] and I was still on drugs and I was still an alcoholic but I cleaned up right away. I've got bad anxiety so I used marijuana to keep myself clean from other drugs and I would say I was [at this Site] almost every day of the week all the time. (WP7)

In Phase One a main findings was that the women participants (mothers that had experiences with the child protection system) all found their drug and alcohol counselor to be very beneficial, but the data did not explain what made drug and alcohol counselor helpful for the women. Therefore this was a focus for Phase Two. Although a number of the women identified their counselor to be helpful, women found a number of other staff members to be beneficial as well because they felt they had trusting, therapeutic relationship with certain staff members. At both 
Sites the staff may have one job title "on paper" but their actual role is much broader, as this participant explained:

So your role, even though everyone has their specific like role whether you be an income assistance worker or a social worker, infant development consultant, you're much more than that role because what overlaps, you know, amongst all the staff is, is just actually having like a personal relationship with the clients right? And, yeah, so I think that's, I mean that's another thing that brings them here right? And what makes them want to stay and actually makes it feel, makes them feel like the services are more accessible? (HCP4- Physician)

This is echoed in the following health care provider's quotation:

My official role is an addictions counselor but I do all sorts of things as most people here do. My background is also in psych nursing so I do a lot of mental health and addictions combined so concurrent disorders. My role here mainly is to provide supportive counseling, deal with crisis management. Basically what we all do build relationships with clients so that they can trust enough to come in here and seek the services that they want. Strong emphasis on having it women-centered here so that they choose what they want. (HCP8- Drug and alcohol counselor)

As many of the women who access Site A and Site B have little or no family or supports, at times the role of the staff is to be someone the women can talk to, a simple but crucial role, as this participant explained:

I cook for them and I'm there when they need hugs or when they need me to hold a baby. I'm there when they need advice on anything in life. I have close clients that come into 
my kitchen and do a job. They prepare for the job, for the world. (HCP6- Peer support worker)

As discussed, the majority of the women participants identified their drug and alcohol counselor as beneficial to their well-being. Additionally, because at both Sites most of the health care providers' roles are multifaceted, outreach workers, peer support workers and nurses also provided counseling. Here a number of the women indicate specifically how they found their drug and alcohol counselor to be beneficial:

My [drug and alcohol counselor] has stepped out of her way when I first started at recovery I could phone here and say man I feel like using, I feel like using. She'd get on her phone or jump in her car and come and see me where I was like really supportive and that's what made me feel like this is my family now. Because I don't have that family but these guys have become my family and I'm, you know, I can phone here and ask for a band aid and they'll help me... (WP4)

This next participant explained that the benefit of the relationship does not necessarily have anything to do with substance use. What was beneficial for her was having someone she could talk with about the things going on in her life:

... [L]ike if something is bothering you or the social worker like it just takes stuff off your chest. Like if you're feeling pissed off or emotional or happy or anything you just talk to her, it's not just about drugs and alcohol it's like all the time like, we just talk about stuff nowadays. Because I don't do drugs and I haven't drank for a long time so she's like we just like talk about [my] social worker, or like "oh my kids are sick" or just whatever that's happening that week we'll talk about it. And it just lifts off some of the 
stress like I'll just say [son's name] is in the hospital. And then like I'll just tell her like how he's doing and like it just makes me feel a little bit better (WP6)

In this last excerpt the participant explains how helpful it is to be able to speak freely without being judged:

... $\mathrm{J}]$ ust getting stuff out that had been bottled in for years and whenever I have problems today I know that I can turn to my drug and alcohol counselor and be honest and truthful and without her judging me. And she doesn't put me down or even I see her, drug addict come in here and they're still using and flailing around and she tells them they look good. And I'm like wow, to me that person doesn't look good right but, you know, and then I asked her one day and she's just like well if you make a person feel better about themselves, that might give them in their minds that they have a chance, oh I'm looking good today, I'm doing well (WP7).

In order to empower women, healthcare must be seen as a choice; health care providers should not try to force women to participate in services in which they are not interested or for which they are not ready. Furthermore, there should be no judgment from health care providers if pregnant or parenting women choose against accessing certain medical services. For many pregnant and parenting women who have complex socio-economic life circumstances, feeling safe and having choices when it relates to their bodies is paramount. Additionally, from the participants' stories, it appears that women are less likely to access health services if they have not first secured the basic human needs, including food, safe and secure housing, adequate income and transportation. 


\section{Educating Healthcare Staff towards Culturally Safe Care}

All of the women and health care providers shared examples of experiencing or witnessing racism and discrimination within mainstream healthcare services and in other bureaucratic systems such as the child protection system. At Site B, the staff received an interactive educational presentation about the history of the residential school experience. This education was presented by an Aboriginal woman, who asked the staff to role-play various people and explained how the Department of Indian Affairs (DIA) came into the homes of Aboriginal people and took the children and their belongings. The Site B participants found this to be extremely beneficial; therefore, providing education in the hospital environment and other healthcare agencies seems to be necessary, as this participant explained:

And so I do think those prejudices do come out right even if, and again it can be really subtle I think. And how do you address it I mean, people say well you just do the [cultural sensitivity] training, it depends on I think it depends on the kind of training as well, I think it needs to be directed by, you know, Aboriginal people...And we talked a bit about as well the child protection system and the fact that it came from her personally and her own personal experiences. I mean obviously it affected us immensely I mean it was something and I think that something, if that were provided in the Ministry of Children and Family Development or whether it be like to nurses, I think that kind of training would get across a lot more than what's usually offered... (HCP5- Child protection social worker)

This participant explained that although the healthcare education curriculum often included cultural sensitivity and related approaches, in her view, this was sometimes lost once people were in the workplace: 
... [W] hen I was at school completing my education [we] did all this work on sort of antioppressive social work and you feel you have this understanding of sort of how people are marginalized and being aware of your own personal biases... But then you go into the work force and you realize you're just faced with it, yeah, like you just feel like that's the system. I don't know there's just this culture within all of these [fields], whether it be social work or the medical field, where even when in front of clients, often it's not in front of clients that these things are said, but it will even come out amongst staff in private when they're just talking to one another right? And obviously if it's coming out then it's a prejudice that they have and it will transfer, in one way or another it does transfer off to the clients and patients... (HCP5-Child protection social worker)

One participant explained that it is not just Caucasian people who are uninformed about the history of colonization of the Aboriginal people and therefore this education should be given to all people:

...[W]e have a whole generation [of Aboriginal people] who has no clue about residential school and what it's done to our people, meaning their grandmothers and great grandmothers and great, great grandmothers, they're not aware of it. So they just see all these Aboriginal people, these wounded Aboriginal people like oh yeah, they're just drunk Indians. Well, no, do you know what happened to your mother; do you know what happened to your grandmother? And when they become aware of what took place and why there are so many people in [on the streets] it's like wow, holy crap I get it like oh my god. I had a family member say to my aunt in front of me "why didn't you go to residential school because we would be getting money right now." And I was like oh my god, are you for real, do you have any clue? And so she's like so they went to a school 
like we all went to school. And I'm like no, no, no, then I sat with my cousin and had an extensive discussion about this is what happened to Aboriginal people. And she's like I had no clue, so it's staggering and mind boggling how even as Aboriginal people we do not know what took place to our people. (HCP7- Family Aboriginal outreach worker) All health care providers would benefit from an understanding of the history of Aboriginal people and colonialism to help reduce the ongoing racism and discrimination experienced by many Aboriginal people.

Another common theme from Phase One related to a lack of what would be considered a family-centred care approach. Specifically, there seemed to be limited parenting support services for male partners. Thus in the interviews I asked the health care providers how they thought we could better serve male partners or fathers who are also involved in the child protection system. This appears to be an extremely complex issue. Some participants thought this was a gap in the system while others believed that the system has far more supportive services for men than for women. However, the majority of health care provider participants thought that supportive parenting services were very limited for Aboriginal men involved in the child protection system, as this participant explained:

Well I think making sort of making a men's [parenting program], because there's a residential school situation because of the historical happenstances for everyone on the reserve, you have a whole group of men who have never been taught to be good fathers. You know some of them have but overall, and these men who have never been shown in the past to be a good father and how to be a good father, some of them had absent fathers, some of them had abusive fathers so to give them services like elders, like a [parenting program] for men, would help them to start to relearn those. And early in the pregnancy 
to help them understand the changes their partner is going to go through. The expectations of what their role will be and how they can support their partner would be really valuable to men. Because a lot of them they want to be involved but don't know how and some of them they've just not had the role models necessary to understand what it means to be involved. (HCP4- Physician)

A number of the health care providers thought parenting programs should be created for men and that when possible (when there is not a concern of violence towards either partner), they could be included in the programs that women access, but also that there should be a separate agency for fathers to access, as this participant explained:

[S]ometimes we [talk about] how there needs to be a low barrier services for men, right?

You know we always welcome partners as long as they're appropriate and the woman wants that male partner here, then they can have lunch with them here and hang out. But, I mean I agree like a lot of the times a concern will be violence right in the home, domestic violence. And a lot of the men have their own trauma issues from the past. And they have their own issues with housing and drug and alcohol use. And they don't actually have any sort of access to services delivered in the same way as it is at Sheway, which is sort of a drop-in model, wrap around service right. (HCP5-Child protection social worker)

Although the issue of including fathers or partners is quite complex, providing a family-focused approach when it is safe and when the parents agree to it seems most beneficial to the children. However, most of the health care providers agreed that their main focus is always the mothers or the pregnant women, as this participant relayed: 
...T]he whole philosophy that we have about keeping families together has to include the dads, and trying to support them to become as healthy as possible and good dads and good partners. And there are some parenting programs and quite often partners will attend them... Because we certainly know that most of the men that we deal with had had for want of a better word a hellish life and have suffered a lot of abuse. And so to try and support them change into, to be better dads than what they had and all that stuff. But I know that there's a gap that we have but it's actually vital that it's part of our whole philosophy and our whole programs. But quite often, you know, the focus is on the mom and the baby and I suppose that's true in healthcare generally... (HCP1-Registered Nurse)

Thus it is apparent from the data is that a number of Aboriginal men and fathers are dealing with similar socio-political and historical issues, which also need to be addressed to promote health and well-being in the family unit. Therefore, more services that foster life skills and parenting skills may be beneficial for men as well.

\section{More Supportive Services}

As discussed earlier, more supportive services are required than what are currently available for pregnant and parenting women with complex socio-political and economic challenges. This includes the need for safe housing with $24 / 7$ supervision for mothers who require additional support for a period of time. Additionally, outreach work seems highly beneficial for women living with very limited resources. Outreach is a significant part of the work done by health care providers at both Site A and Site B. This can include driving women to appointments, bringing women food, attending mediation, sitting in on physician appointments, or providing nursing care out in the community (in shelters, in their homes, in detox, at the 
hospital, even sometimes in jail). This is one way that health care providers establish a trusting relationship with the women; however, this is not always well-received by other health care providers. But as this participant explained, some clients need this extra help because they do not have support otherwise:

... [W] go to [the women's] home or pick them up or take them to their doctor's office or we'll take them to their ultrasound or we'll take them to the hospital because it's important, you know, it's important that they get that and the people are a bit apprehensive and especially going to the hospital or the health unit they seem to be intimidated by these big institutions, and for good reason, the history isn't good... (HCP1-Registered Nurse).

Outreach work reduces some of the barriers faced by the women. Although the women participants did not refer to it as outreach, several women shared stories of the staff from Site A and Site B going "out of their way" to help them access certain services, and one of the things they really appreciated was any type of assistance with transportation.

Last, one of the major differences described by the health care providers of Site A and Site B is that the doctors employed at Site A do not work in labor and delivery. At Site A the pregnant women are referred to community doctors for labor and delivery, while at Site B the doctors also work out of a local hospital's birthing program and therefore can perform this service for their clients. As one health care provider participant from Site A explained,

The thing which is too bad here at the clinic [is the doctors] don't deliver our own patients so we have to refer to delivering doctors. We do have doctors that deliver babies but they deliver them from their other office. They work here very part-time so they don't deliver our patients. So ideally that would be the way to go is to have an all in- 
house service... [Because] you know, we've had some experiences of [women] being sent to other doctors and maybe them contacting the Ministry or doing stuff in a slightly different way than we would have done. (HCP 1-Registered Nurse)

A health care provider from Site B explained how key it is for their clients to have a relationship with the physician who will deliver their baby:

A very huge thing about our doctors [at Site B] is that they work at [a nearby hospital] so the women get continuity of care and they're not meeting somebody totally new. They might meet somebody new but then they'll see one of our doctors so it's not totally scary for them. (HCP8- Drug and alcohol counselor)

To these two health care providers, having physicians who know the women and understand their life circumstances is optimal for continuity of care at the time of labor and delivery.

\section{Summary of the Findings}

The women and health care providers who participated in Phase Two shared their rich stories and perspectives in relation to healthcare and access to healthcare when child protection services are concurrently involved in pregnant and parenting Aboriginal women's lives. The insights gained from the participants have highlighted the complex intersections of poverty, violence, substance use, racism, discrimination, colonial policies, the child protection system and health for Aboriginal women and children. In the next chapter I discuss the main findings from Phase One and Phase Two. 


\section{CHPATER 6: DISCUSSION AND RECOMMENDATIONS}

\section{Introduction}

The purpose of this study was to explore how the threat of child apprehension impacts Aboriginal women's and children's experiences accessing healthcare services. Throughout this process I also gained a greater understanding of how the child protection system in B.C. intersects with healthcare. In Phase One and Phase Two I analyzed in-depth, interview transcripts of (i) Aboriginal mothers, all of whom had experiences with the child protection system and (ii) health care providers with expertise in working with pregnant and parenting women who are likely to be investigated by the child protection system. The analysis also included accompanying fieldnotes taken from the interviews in both Phases.

The sample of pregnant and parenting Aboriginal women recruited for this study was small ( $\mathrm{n}=9$ ); however, the context of the women participants' lives was similar to what is known in the literature about the socio-economic and ethnocultural characteristics of mothers often involved in the child protection system (lone-parent households, low income, and Aboriginal ethnicity). The women participants provided great insight and rich perspectives on this topic. I begin this chapter by discussing how the threat of child apprehension impacted the women participants' experiences with mainstream healthcare services.

\section{How does the Fear of Child Apprehension Impact Experiences with Mainstream}

\section{Healthcare?}

There is an abundance of literature highlighting poorer health outcomes for Aboriginal women in comparison to non-Aboriginal women in Canada (Canadian Institute of Health Research, 2008b; Adelson, 2005; Bourassa et al., 2004; Canadian Institute of Health Information, 2004). There is also a large amount of data indicating a disproportionately higher 
number of Aboriginal children in government care (Farris-Manning \& Foster, 2003; National Collaboration Centre for Aboriginal Health, 2009-2010a; Blackstock \& Trocmé, 2005). The central goal of this study was to better understand how these two distressing factors intersect and impact Aboriginal women's experiences with mainstream healthcare agencies. The findings from this study did not give one uniform perspective; however, several of the participants agreed that mainstream healthcare agencies such as hospitals are environments where parenting practices are observed and, for Aboriginal people, quite often judged. The women described a fear of being judged by health care providers in relation to their parenting, voicing concern about the influence the nurses' judgments could have on child protection investigations.

The participants involved in this study agreed that when it comes to the health and wellbeing of children, the fear or threat of child apprehension and the fear of judgment have little impact on Aboriginal mothers' decisions to seek available medical services for their children. However, the fear of being judged by mainstream health care providers impacted many of the women's experiences within mainstream healthcare agencies. The outcome of this fear had particular effects on the quality of interactions at the individual level between Aboriginal women and health care providers. Additionally, negative experiences with hospital staff also deterred some women from seeking necessary medical services for themselves. Thus the fear of being scrutinized acts as a barrier preventing some Aboriginal women from receiving necessary, quality healthcare. This fear of judgment occurs within a context of economic a political disadvantage faced by many Aboriginal women. Thus the dynamics of the influence of the threat of child apprehensions on health cannot be discussed without taking into account: the interpersonal discrimination experienced by Aboriginal women; the structural poverty and discrimination experienced by Aboriginal women; the legislative authority of the child 
protections system and its enactment with Aboriginal people; and, the lack of support for people living in poverty, particularly Aboriginal women. Following the discussion, I offer some recommendations for policy makers, leaders and practitioners working in various mainstream healthcare agencies and institutions.

\section{Experiences of Interpersonal Discrimination in Mainstream Healthcare}

Racism, sexism and discrimination toward Aboriginal women through sexist and oppressive government policies has been well documented in the literature (see Bourassa et al., 2006; Kubik, Bourassa, \& Hampton, 2009; Castleden, Crooks, Hanlon, \& Schuurman, 2009; Ship \& Norton, 2001). Discrimination toward Aboriginal women at the wider socio-political and economic level filter through and impact individual level interactions One striking aspect of the findings was that all of the women participants described hospital nurses as conveying overtly racist and judgmental behaviors toward them. In particular, the women described examples of hospital nurses critiquing and judging their parenting practices. Their experiences of discrimination from hospital nurses impacted some participants' abilities to visit their children upon subsequent hospitalizations, as they were unwilling to subject themselves to a similar experience again. Consequently, a number of the women described experiencing further judgments by hospital staff for their inability to stay at the hospital throughout their child's subsequent hospitalizations. These findings are not unique; Browne and Fiske (2001) presented similar findings from a study that examined First Nations women's encounters with mainstream healthcare. The women from Browne and Fiske's study also reported experiencing discriminatory and judgmental attitudes and behaviors from mainstream health care providers. Poudrier and Mac-Lean shared similar findings in a study examining Aboriginal women's experiences with breast cancer (2009). Poudrier and Mac-Lean described "invalidating 
encounters" such as racist remarks from health care providers toward Aboriginal women. Racism or discriminatory care from nurses is particularly deleterious since nurses are frontline health care providers in the hospital setting and make up a large percentage of the health care providers within the hospital environment. Nurses are representative of a health care organizations approach to care, and set a tone and expectations for people accessing care.

One of the women participants from Phase Two expressed her belief that "the hospital staff don't want to deal with Natives." This perception of mainstream health care providers was conveyed by several of the women. Another woman provided an example of being discharged prematurely from the hospital and, when she asked why, the staff called security to have her removed. This woman reported that she was also informed that she was "in a hospital not a hotel," yet she was homeless and consequently sent out to the streets. This woman's story is just one example of overt racism and discrimination described by the participants. At the individual level, racism and discrimination towards Aboriginal people in mainstream healthcare agencies such as hospitals must be acknowledged by policy makers, educators and leaders as an ongoing problem that must be addressed.

Assumptions about Aboriginal people and Aboriginal culture have the potential to influence nurses' practice (Browne \& Varcoe, 2006; Browne, Varcoe et al., 2009). The concept of culture was discussed earlier in this study (see Chapter 2); however, I come back to it here because several participants described experiences where they reported feeling particularly scrutinized or judged because of their Aboriginal ethnicity. Browne and Varcoe discuss the ideology of culturalism: "the complex practice and ideology that uses popularized, stereotyped representations of culture as the primary analytical lens for understanding presumed differences about various groups of people." (p.6) 
Culturalism or culturalist definitions of culture can be problematic because they define culture as something that is static; focused primarily on shared values, traditions, beliefs and customs (Browne \& Varcoe, 2006). This narrow view of culture sees certain characteristics of various ethnocultural groups as 'cultural traits' without considering the complex socio-political and economic factors that shape people's lives. For example, social constructions of Aboriginal mothers as "neglectful" have been displayed in the media and other public forums, yet little attention has been given to the wider socio-political and economic factors that have led to Aboriginal women experiencing higher rates of poverty and poorer health than non-Aboriginal women (Browne \& Varcoe). Nurses have been trained to understand culture primarily through a culturalist lens, which can be problematic when it leads to stereotypical thinking in which nurses assume that certain behaviors are 'cultural' and miss the wider socio-political and economic factors that shape people's life circumstances. Thus one of the recommendations from this study is to educate health care providers such as nurses with a more critical understanding of culture.

\section{Structural Poverty and Structural Discrimination}

\section{Structural Poverty}

Throughout this study, poverty has been at the forefront of concern as it relates to adverse health outcomes and also as a key factor shaping child protection investigations. All of the women who participated in this study were connected to Site A or Site B; these Sites provide comprehensive services, including a number of healthcare services and various social supports. Consequently, most of these women had assistance in reducing some of the socio-economic barriers faced by pregnant and parenting women living in poverty (e.g., they had assistance in securing food and available housing; legal aid; and assistance with attaining material necessities such as strollers and cribs). However, even with the support of Site A or Site B, many of the 
women reported ongoing challenges securing the basic needs for survival. For example, one participant was living in a one-bedroom apartment with four children. The apartment was arranged through Site B as a temporary housing solution, but at the time of the interview the woman was "stressed out" as she was looking without success for a more spacious place to live. This participant had moved a number of times in previous years. Two of her children had recurrent respiratory infections throughout their first years of life, including her ten- month-old son, who had been hospitalized more than ten times that year for acute respiratory infections. Her children's health issues were affected by their living conditions. Children that live in substandard living conditions (e.g. damp, cold or moldy homes) are more prone to respiratory infections such as bronchitis and asthma as well as other chronic illness (Carter \& Polevychok, 2004; Krieger \& Higgins, 2002; Landrigan, Rauh, \& Galvez, 2010).

The data from the health care providers' interviews emphasized a need for more social supports for populations living in poverty. The available social supports were not meeting the needs of the community in which the Sites are located. Further, the data from Phase One and Two indicated that pregnant and parenting women living in poverty need more assistance securing adequate food, shelter, and income - the social determinants of health. The health care providers described how funding cuts have resulted in the closure of many community programs that attended to the social determinants of health, such as food banks and drug and alcohol treatment programs. The closure of these programs has been problematic specifically for pregnant and parenting women, as both Sites rely on them in arranging food, shelter and other social programs for women. The women participants described several factors impacting their access to healthcare which were predominantly structural inequities, such as poverty, racism, and 
discrimination. Therefore, the first step towards improving the health status of Aboriginal women and children is to address inequities relating to the social determinants of health.

\section{Structural Discrimination}

Discrimination and racism were expressed as ongoing factors that shaped the women participants' experiences with healthcare and the child protection system. Structural racism occurs when certain groups of people are discriminated against or disadvantaged through policy and economic decisions (Browne et al., manuscript in review). A case in point of structural discrimination is the allocation of funding to First Nations child welfare agencies. Originally the study was not focused on First Nations child welfare agencies; however they are discussed here to illustrate broader issues of structural discrimination.

As explained in the literature review, there are a number of Aboriginal appointed child welfare agencies within B.C. and Canada, which are operating at various levels of authority. Although child protection is primarily a provincial government responsibility, the federal government plays a more central role in funding child welfare agencies for First Nations people. This is due to the federal government's responsibility on issues related to First Nations people. In 1991 the Canadian federal government created a program known as Directive 20-1. Directive 20-1 was designed to fund First Nations child welfare services throughout Canada for those living on reserve. However, the funding received by delegated First Nations child and family service agencies is $22 \%$ less than what is given to mainstream agencies (National Collaborating Centre for Aboriginal Health, 2009-2010b). There are a disproportionately higher number of First Nations children in foster care and yet First Nations child welfare agencies operate with significantly less funding. Thus one of the groups with the highest need of support must manage with fewer resources than what is available for mainstream agencies (such as MCFD). 
Blackstock and Trocmé presented findings from Manitoba's 2003 provincial data, which indicated that "...although Aboriginal children comprise 70\% of the children in care in that province, Aboriginal families were benefiting from only $30 \%$ of the child welfare family support budget" (2005, p. 28). Manitoba has been considered the most supportive province in relation to child welfare issues for Aboriginal people; however these findings indicate that the province allocates significantly less funds to Aboriginal child welfare agencies (Blackstock \& Trocmé).

The move towards Aboriginal child welfare agencies is beneficial for a number of reasons. For instance, creating Aboriginal child welfare agencies aligns with the First Nations goal of self-government (National Collaborating Centre for Aboriginal Health, 2009-2010b). Additionally, these agencies are dedicated to preserving Aboriginal families and communities through a commitment to keeping Aboriginal children in Aboriginal communities and homes, but this is only possible with adequate funding. The separation of children from their families can have serious consequences on children's health and well-being (Cull, 2006). Mainstream social institutions, such as provincial child welfare services have failed to support Aboriginal children and families (Monture, 1989; Harding, 2006; National Collaborating Centre for Aboriginal Health, 2009-2010c). If the provincial and federal governments are truly committed to keeping Aboriginal families and communities connected there must be more equitable funding allocated to Aboriginal children involved in the child protection system.

\section{Legislative Authority within the Child, Family and Community Services Act}

Several women participants expressed frustrations in relation to their child protection workers. Most participants thought that child protection social workers wield a significant amount of power, which was at times described as "being held over [the women]." The power that some child protection social workers possess is their authority to enact the Child, Family and 
Community Services Act, which as stated previously, is the legislation for the Ministry's child protection services. MCFD states that child protection social workers are to take the most appropriate action that is least disruptive for the child; however, when necessary for the child's safety, a child can be removed from its parents by a child protection social worker (Ministry of Child and Family Development, n.d.).

A child protection social worker's legislative authority requires child protection workers, at times, to make custody decisions for a child. If a child protection social worker investigates a case and decides that a file needs to be opened, the next step is deciding what interventions will be taken (e.g., supportive services, supervising the child or removal). Although these decisions are made in collaboration with supervisors, the decision is largely based on an investigation carried out by one child protection worker. The challenge is that the decision of what is best for the child (removal, supervision or supportive services) is largely a judgment call. Some child protection social workers may feel more comfortable with families in less than ideal situations (e.g., inadequate housing conditions) while others may feel less comfortable, in part because the child protection system has been scrutinized for how cases have been managed in recent years (Pivot Legal Society, 2008).

The CFCSA was passed in BC in 1994 but did not come into force until January of 1996. At its inception, this legislation was seen as potentially improving the conditions for families involved in the child protection system. However, as the CFCSA was coming into legislation, Justice Thomas Gove's report was released (Pivot Legal Society, 2008). It has been suggested that one of the results of the Gove inquiry was a shift in practice away from family preservation to a more conservative approach, focused more narrowly on the safety of the child (Pivot Legal Society; Hughes, 2006). The Gove inquiry in 1994 examined the death of Matthew Vaudreueil. 
Matthew died of asphyxia, his emaciated body found beaten (Pivot Legal Society). Matthew and his mother had been involved in the child protection system throughout his life and Gove's inquiry into Matthew's death highlighted clear evidence that the case had been poorly handled (Pivot Legal Society). The concerns from the Gove inquiry of the child protection system included: a lack of staff hours, poor staff training and uncoordinated services (Pivot Legal Society).

The Gove report also stated that a family-centred approach to child protection service delivery in $\mathrm{BC}$ was problematic because front-line child protection social workers do not have sufficient qualifications, training, and skills required to decide when children are at significant risk in the care of their parents ( as cited by Pivot Legal Society, 2008, p. 17). At the time of the Gove inquiry, the child protection system and child safety became a public concern and a spike in child apprehensions ensued (Hughes, 2006, p.5). Throughout the late 1990s there were some attempts within the child welfare system to preserve families and cultures, such as the creation of Aboriginal child welfare agencies; however a high percentage of child protection investigations, particularly for Aboriginal children, continued to result in child removals. This seems to be partially the result of inadequate funding allocation to child welfare systems, resulting in child protection social workers operating with heavy caseloads, and few available supportive services to offer parents or families. However, the decisions by child protection social workers to apprehend may also be impacted by stories such as Matthew Vaudreueil, which left the public questioning who was responsible for the death of a child well known to the child protection system.

Based on the Phase Two analysis, as well as what is available in the literature, decisions about child custody are partially shaped by how the child protection social worker interprets the 
CFCSA. Some child protection social workers are more willing to make accommodations for less-than-ideal family circumstances and will help the families create safety plans around their issues; while others are less willing to make accommodations for families because that child protection worker is ultimately responsible for the child's well-being. Custody decisions also appear to be shaped by the relationship between the mother and her child protection social worker, as well as the mother's overall capacity to parent (which includes her resources and supports). The consequence of these various factors that shape child protection social workers' decision-making in custody matters is a lack of consistency from worker to worker (or even from case to case). The participants from Phase Two found the lack of consistency to be problematic, as the women had trouble determining what expectations they must meet to keep or regain custody of their children.

\section{Lack of Supportive Services}

In both Phases the participants expressed challenges with the limited supportive services available for women and families who rely on social assistance. Due to government cuts to social programs, mothers are increasingly expected to gain the skills and resources necessary for parenting with little if any assistance (Brown, 2006). The lack of supportive services is not just a concern to Aboriginal appointed agencies; funding is generally insufficient for child protection services in Canada. The result of inadequate funding has led to a risk management approach to child protection in Canada. As Brown explains, risk management approaches focus on narrow, cost-conscious programs that disproportionately affect individuals already living on the margins of society (p.354). Site A and Site B provide low barrier, comprehensive medical and social services to people who are generally living at the margins of society. These Sites both particularly focus on women's health and whenever possible they are dedicated to keeping 
Aboriginal women and children together, as family connectedness has a positive impact on health and well-being (Cull, 2006). However this is not possible without sufficient social support.

An unanticipated finding from Phase One, subsequently explored in Phase Two, was the lack of supportive services available for fathers or partners. For example, Site B is a program that serves pregnant and parenting women and children; they will allow partners to join in on the mother's various programs, but the focus is always on the women. The health care providers did not agree on the best way to incorporate partners or fathers into programs. Two health care provider participants from Site B thought that generally the community in which they are located had more services for men than for women, but when I asked what those services were, they described things such as health clinics, methadone programs and detox programs rather than parenting programs, counselors, or anger management classes. Other health care providers saw the limited social services available for fathers or partners as a 'gap' that should be addressed. The main consensus was that there are few services available that provide low barrier health and social services for men and women, while men are particularly lacking parenting programs and related services.

The participants from this study highlighted challenges around providing services that are "family-centred", involving both parents. The main challenge is when a woman's partner is known to be violent, as often there is a court order that prevents the partners from seeing each other. However, services need to be available for Aboriginal men so they also can deal with their issues stemming from the history of government intervention into Aboriginal people's lives. 


\section{Recommendations}

\section{Inter-Sectoral Efforts on Reducing Poverty}

. The challenges for many Aboriginal women trying to parent while living at or below the poverty line have been discussed throughout this study. Aboriginal women are far more likely to live in poverty than other Canadian women (Pendakur \& Pendakur, 2011; Bourassa et al., 2004; Kubik et al., 2009; Browne, Fiske, Thomas, 2000). The statistics on poverty for First Nations children in Canada are particularly disturbing. One in every four First Nations children is growing up in poverty (Rothman, 2007). As stated previously, there is a wealth of research highlighting the association of poverty to poorer health outcomes (National Council of Welfare Reports, p. 8; Canadian Institute for Health Information, 2008b; Reutter, Veenstra, Stewart, Raphael, Love, Makwarimba, \& McMurray, 2006). The provincial and federal governments must recognize the effects of poverty on the health of Aboriginal women and children and create policies that are committed to addressing the inequities in relation to the social determinants of health.

An area that cannot be ignored by policy makers is the need for more social housing for those in need. The lack of affordable housing not only impacts the health of individuals, it also impacts women's ability to provide and care for their children. Cohen-Schlanger, Fitzpatrick, Hulchanski, \& Raphael (1995) surveyed social workers from Toronto's child welfare system to examine the relationship between housing problems and decisions around placing children into temporary care. "In $18.4 \%$ of the cases, the family's housing situation was identified by the family service worker ${ }^{10}$ as "one of the factors that resulted in temporary placement of a

\footnotetext{
${ }^{10}$ The term family service worker refers to a “...professional social workers who visit families in their home as a routine practice, and have first-hand information of a family's housing circumstances. Therefore, these social workers are particularly knowledgeable about the major factors affecting the families and children they work with." (Chau et al., 2001, p.2)
} 
child/children into care." (Schlanger et al., p.554). Similarly, one of the key findings from Chau, Fitzpatrick, Hulchanski, Leslie, \& Schatia indicated that in one out of five cases, housing was a factor that resulted in the temporary placement of a child into care (2001, p. 3). These findings are not meant to imply that adequate and affordable housing will directly result in the prevention of child apprehensions; rather, affordable, safe and accessible housing may help to decrease the number of children removed from homes, provide stability within a family's home environment, and decrease housing-related delays that factor in the return of children to families (Chau et al., p.9). The lack of social housing available in Canada intersects with the child welfare policies in a paradoxical manner, “...in that secure housing is often a condition of maintaining custody; yet having custody is a requirement of obtaining social housing" (Jategaonkar \& Ponic, p. 2). The housing crisis highlights one of the many challenges for many pregnant and parenting (or trying to parent) women living in poverty.

Housing issues impacted a number of the women participants in this study. The complication of poverty as it relates to housing is just one example of how Aboriginal women and children are particularly impacted by restrictive government policies, which perpetuate substandard living conditions for many Aboriginal people. This can only change through a commitment from policy makers to focus on reducing the social inequities experienced by Aboriginal people in Canada.

\section{Improving the Process of Child Apprehensions in the Hospital Environment}

I begin this section by stating that the paramount goal and recommendation from this study is to increase and improve services available for parents and families so children can remain with their parents whenever possible. However, since at this point in time child removals are still a regular occurrence, it is imperative to address the process in which the removal is 
carried out so as to prevent any added and unnecessary trauma. Having a child removed from a parent's custody was described by a number of the participants as highly traumatic. From the perspectives of several participants, the current high percentage of children in government care is sometimes seen as an extension of the residential school system. As it was explained by one participant, children are removed from their parents because an authoritative figure views it to be in the best interest of the child. While the child remains in placed in foster care, the parents often have little to no contact with their child.

The women's stories of their children's experiences in foster care were often quite devastating. Two women (one from Phase One and one from Phase Two) relayed positive relationships with the foster parents and themselves but these cases seem to be the exception. Further, regardless of the positive relationship between the participants and the foster parents, both women were fighting to regain custody of their children.

There are times when the only "safe" option for the child is to be removed from the parent. However, often the women participants in this study described circumstances where if the women had been provided with some supportive services an apprehension may have been preventable. Further, the expectations of some of the women's child protection social workers seemed difficult to live up to for any person, let alone a single mother, living in poverty and with no extended family support. However, if and when a child needs to be removed from their parent it must be carried out in a way that attempts to diminish the trauma of the experience for the mother. As one of the participants said,

... [P]lan around it if [the Ministry] is going to remove.... [the mom] doesn't have to watch and [health care providers] don't have to sit there and judge [the mom] because 
that's what the nurses were doing, they were judging her. Why couldn't they see a broken heart; they couldn't see the tears. (HCP6)

Health care providers working in maternal-child health areas must advocate for the dignity and the respect of women clients. One of the health care providers relayed a quotation that she asked me to write out, "If I have not seen you at your worst I do not deserve to see you at your best." I have analyzed the meaning of this quotation as it relates to the topic of this study extensively, and my interpretation of it is that people are told endlessly that they must respect health care providers in mainstream healthcare agencies, and if they do not, they may not receive services. Yet the women in this study described several experiences of being demeaned or discriminated against from the very people who are demanding respect. One of the women participants said, "[I]f [health care providers] want some respect how about [health care providers] give me a little respect, it goes both ways." (WP1). The removal of a child must be understood as a great loss, which requires advocacy and support, not judgment.

\section{The Provision of Culturally Safe Training to Health Care Providers}

Providing education about culturally safe care as well as indigenous cultural training to health care providers is essential. As discussed previously, the women participants all described experiences with overt racism, discrimination and prejudice in mainstream healthcare agencies. Part of the problem is that when health care providers hear their colleagues making racist statements others do not feel equipped or safe enough to challenge such remarks (Browne \& Varcoe, 2006). One possible solution to this challenge is indigenous cultural competency training (Browne, Varcoe et al., 2009; Browne \& Varcoe; Browne \& Fiske, 2001), which is available through the health authorities in British Columbia. The training is comprehensive; it provides the 
learner with an overview of the history of Aboriginal people in Canada and it also allows for discussions around dealing with racist or judgmental colleagues.

As discussed in Phase Two, the staff at Site B received an interactive education seminar aimed at informing the staff about the history of the residential school system and its ongoing effects on Aboriginal peoples' health and well-being. This education session was viewed positively by the staff, as a high percentage of their clientele are Aboriginal. It is essential for health care providers working with Aboriginal people to be informed about the history of colonialization and the intergenerational impact of colonialism. Health care providers who lack this knowledge about the history of relations between Aboriginal people and the dominant Western society risk providing care that is shaped by negative attitudes and assumptions of Aboriginal people that are embedded in our society (Browne \& Varcoe, 2006; Browne, Varcoe et al., 2009).

The concept of cultural safety was introduced in the literature review. Cultural safety is a critical form of inquiry, which pays attentions to power imbalances and inequitable social relationships in healthcare (Browne, Varcoe et al., 2009, p. 168). Therefore a culturally safe approach to care acknowledges power imbalances between certain groups of people, and aims to prevent 'unsafe' situations, where a member of one ethnocultural group feels demeaned or disempowered by people from another group (Browne, Varcoe et al., 2009).

Practicing health care providers and future health care workers must be equipped with the tools needed to deliver healthcare that is culturally safe. This includes indigenous cultural training, cultural safety education, and an understanding of the current socio-economic and political inequities experienced by certain groups, such as Aboriginal women and children. If Aboriginal women do not feel safe in mainstream healthcare agencies they may avoid using them 
(Browne, 2007; Heaman, Green, Newburn-Cook, Elliot, \& Helewa, 2007; Heaman, Gupton \&Moffatt, 2005). Diminishing the barriers to healthcare services through providing culturally safe care is a vital step towards reducing the health inequities experienced by many Aboriginal peoples in Canada.

\section{Conclusion}

The women participants' experiences of accessing healthcare services were shaped by a number of structural inequities including poverty, racism, discrimination, violence, and oppressive government policies. The threat of child apprehension is yet another factor that shapes the health status and well-being of both Aboriginal women and children. Higher rates of poverty, social exclusion, racism, and socio-economic disadvantage experienced by many Aboriginal people impacts all aspects of life including health and family connectedness (Blackstock \& Trocmé, 2005). In order to address these problems, policy shifts and government initiatives must focus their attention on culturally supportive, community-driven programs aimed to reduce the social and economic inequities experienced by many Aboriginal populations. 


\section{REFERENCES}

Adelson, N. (2005). The embodiment of inequity: health disparities in Aboriginal Canada. Canadian Journal of Public Health, 96 supplement 2, S45-S61.

Anderson, J.M. (2002). Toward a post-colonial feminist methodology in nursing research: exploring the convergence of post-colonial and black feminist scholarship. Nurse Research, 9(3), 7-27.

Anderson, J.M. (2004). Lessons from a postcolonial-feminist perspective: suffering and a path to healing. Nursing Inquiry, 11(4), 238-246.

Armitage, A. (1995). Canada: the general structure of Canadian Indian policy. In Armitage, A. Comparing the Policy of Aboriginal Assimilation: Canada and New Zealand. (p. 70-99). Vancouver: UBC Press.

Benoit, C., Carroll, D., \& Westfall, R. (2007). Women's access to maternity services in Canada: historical developments and contemporary challenges. In Morrow, M., Hankivsky, O., \& Varcoe, C. Women's Health in Canada: Critical Perspectives on Theory and Policy. Toronto: University of Toronto Press.

Bhabha, H. K. (1994). The Location of Culture. p. 171-197: Routledge: New York.

Blackstock, C., \& Trocmé, N. (2005). Community-based child welfare for Aboriginal children: supporting resilience through structural change. Social Policy Journal of New Zealand, 24(2), 12-33.

Blackstock, C., Trocmé, N., Bennett, M. (2004). Child maltreatment investigations among Aboriginal and non-Aboriginal families in Canada. Violence Against Women, 10(8), 901916. 
Bourassa, C., McKay-McNabb, K., \& Hampton, M. (2004). Racism, sexism, and colonialism: the impact on the health of Aboriginal women in Canada. Canadian Woman Studies, 24(1), 23-29.

Boyatzis, R.E. (1998). Transforming Qualitative Information. (p. 1-28). Thousand Oaks, California: Sage.

British Columbia’s Ministry of Attorney General (2011). Child Protection Mediation Program. Retrieved from www.ag.gov.bc.ca/child-protection-mediation/documents/cpm.pdf

British Columbia's Ministry of Child and Family Development (2012). Protecting Children. Retrieved from http://www.mcf.gov.bc.ca/child_protection/

British Columbia. Provincial Health Officer. (2009). Pathways to health and healing-2 ${ }^{\text {nd }}$ report on the health and well-being of Aboriginal people in British Columbia. Provincial Health Officer's Annual Report 2007. Victoria, BC: Ministry of Healthy Living and Sport. Retrieved from www.health.gov.bc.ca/pho/pdf/abohlth11-var7.pdf

Brown, D. (2006). Working the system: Rethinking the institutionally organized role of mothers and the reduction of "risk" in the child protection system. Social Problems, 53(3), 352370.

Brown, H., Varcoe, C., \& Calam, B. (2011). The birthing experience of rural Aboriginal women in context: implications for nursing. Canadian Journal of Nursing Research, 43(4), 100 117.

Browne, A.J. (2007). Clinical encounters between nurses and First Nations women in a western Canadian hospital. Social Science \& Medicine, 64(10), 2165-2176. 
Browne, A.J. et al. (Manuscript in Review). Closing the Health Equity Gap: Strategies for Primary Healthcare Organizations. From the CIHR funded study, Improving access to primary healthcare: Lessons learned from two urban Aboriginal health centres.

Browne A.J. et al. (2007). CIHR funded proposal: Improving access to primary healthcare: Lessons learned from two urban Aboriginal Health centres. Unpublished manuscript, University of British Columbia, Vancouver, B.C., Canada.

Browne, A.J., \& Fiske, (2001). First Nations women's encounters with mainstream health care services. Western Journal of Nursing Research, 23(2), 126-147.

Browne, A., Fiske, J., \& Thomas, G. (2000). First nations women's encounters with mainstream health care services and systems. Vancouver, BC: BC Centre of Excellence for Women's Health

Browne, A.J., McDonald, H., \& Elliot, D. (2009). Urban First Nations Health Research Discussion Paper. A report for the First Nations centre, National Aboriginal Health Organization. Ottawa, ON: National Aboriginal Health Organization.

Browne, A.J., Smye, V., \& Varcoe, C. (2007). Postcolonial-feminist theoretical perspectives and women's health. In Morrow, M., Hankivsky, O., \& Varcoe, C. (Eds.). Women's Health in Canada: Critical Perspectives in Theory and Policy. Toronto: University of Toronto Press.

Browne, A.J., Smye, V., \& Varcoe, C. (2005). The relevance of postcolonial theoretical perspectives to research in Aboriginal health. Contemporary journal of Nursing Research, 37(4), 16-37.

Browne, A.J., \& Varcoe, (2006). Critical cultural perspectives and healthcare involving Aboriginal peoples. Contemporary Nurse, 22(2), 155-167. 
Browne, A.J., Varcoe, C., Smye, V., Reimer-Kirkham, S., Lyman, J., \& Wong, S. (2009).

Cultural safety and the challenge of translating critically oriented knowledge in practice. Nursing Philosophy, 10(3), 167-179.

Canadian Council of Provincial Child and Youth Advocates (2010). Aboriginal children and youth in care: Canada must do better. Position paper. Retrieved from http://www.gnb.ca/0073/PDF/positionpaper-e.pdf

Canadian Institute of Health Research. (2004). Improving the health of Canadians. (p.73-104). Retrieved from http://secure.cihi.ca/cihiweb/products/IHC2004rev_e.pdf

Canadian Institute of Health Research. (2008a). Institute of Aboriginal peoples' health commemorative report 2000-2008. Retrieved from http://publications.gc.ca/collections/collection_2009/irsc-cihr/MR21-126-2008E.pdf

Canadian Institute for Health Information (2008b). Reducing the Gaps in Health: A focus on Socio-Economic Status in Urban Canada (Ottawa, Ontario: CIHI, 2008). Retrieved from http://secure.cihi.ca/cihiweb/products/Reducing_Gaps_in_Health_Report_EN_081009.pd $\mathrm{f}$

Caspecken, P.F. (1996). Critical ethnography in educational research: a theoretical and practical guide. New York: Routledge.

Carter, T., \& Polevychok, C. (2004). Housing is good social policy. Canadian Policy Research Networks, Research Report F50, Ottawa.

Castleden, H., Crooks, V.A., Hanlon, N., \& Shuurman, N. (2010). Providers' perceptions of Aboriginal palliative care in British Columbia's rural interior. Health \& Social Care in the Community, 18(5), 483-491. 
Chau, S., Fitzpatrick, A., Hulchanski, J.D., Leslie, B., \& Schatia, D. (2001). One in five...Housing as a factor in the admission of children to care: new survey of Children's Aid Society of Toronto's updates 1992 study. A Joint Research Project by the Children's Aid Society of Toronto and the Faculty of Social Work, University of Toronto Centre for Urban and Community Studies, Research Bulletin \#5. Retrieved from http://www.homelesshub.ca/ResourceFiles/Documents/1.3\%20Chau\%20et\%20al.\%20$\% 200$ ne $\% 20$ in\%20Five.pdf

Chuchryk, P., \& Miller, C. (1996). Introduction. In Miller, C., \& Chuchryk, P. Women of the Frist Nations: Power, Wisdom, and Strength (Eds). Winnipeg: University of Manitoba Press.

Cohen-Schlanger, M., Fitzpatrick, A., Hulchanski, J.D., \& Raphael, D. (1995). Housing as a factor in admission of children to temporary care: a survey. Child Welfare, 74(3), 547562.

Cull, R. (2006). Aboriginal mothering under the state's gaze. In Lavelle-Harvard, D.M. \& Lavelle, J.C. Until our Hearts are on the Ground: Aboriginal Mothering, Oppression, Resistance, and Rebirth. Toronto: Demeter Press

De Leeuw, S. (2007). Intimate colonialism: the material experienced place of British Columbia's residential schools. Canadian Geographer, 51(3), 339-359.

Duva, J. \& Metzger, S. (2010) Addressing Poverty as a Major Risk Factor in Child Neglect: Promising Policy and Practice, Protecting Children, 25(1), 63-74. 
Farris-Manning, C. \& Foster, M. Z. (2003). Children in care in Canada: A summary of current issues and trends with recommendations for future research. Child Welfare League of Canada. Retrieved from http://www.nationalchildrensalliance.com/nca/pubs/2003/Children_in_Care_March_2003 .pdf

Fetterman, D.M. (1998). Ethnography: Step by step second edition. In Applied Social Research Methods series vol $17\left(2^{\text {nd }}\right.$ ed.). (p. 34-50). Thousand Oaks, California: Sage.

Fiske, J. (1995). Political status of Native Indian women: Contradictory implications of Canadian state policy. American Indian Culture and Research Journal, 19(2), 1-30.

Fournier, S. \& Crey, E. (1997). Stolen from our embrace: the abduction of First Nations children and the restoration of Aboriginal communities. Vancouver: Douglas \& McIntyre.

Furniss, E. (1999). The burden of history: Colonialism and the frontier myth in a rural Canadian community. (p. 27-52). Vancouver, BC, Canada: University of British Columbia Press.

Greenwood, M. \& De Leeuw, S. (2006). Fostering indigeneity: the role of Aboriginal mothers and Aboriginal early child care in responses to colonial foster-care interventions. In Lavell-Harvard, D.M. \& Lavelle, J.C., Until our Hearts are on the Ground: Aboriginal Mothering, Oppression, Resistance and Rebirth. Toronto: Demeter Press.

Harding, R (2006). Historical representations of Aboriginal people in Canadian news and media. Discourse \& Society, 17(2), 205-235.

Heaman, M. I., Green, C. G., Newburn-Cook, C. V., Elliott, L. J., Helewa, M. E. (2007). Social inequalities in use of prenatal care in Manitoba. Journal of Obstetrics and Gynaecology, 29(10), 806-816. 
Heaman, M., Gupton, A., \& Moffatt, M. (2005). Prevalence and predictors of inadequate prenatal care: A comparison of Aboriginal and non-Aboriginal women in Manitoba. Journal of Obstetrics and Gynaecology Canada, 27(3), 237-246.

Hughes, T, (2006). BC children and youth review: an independent review of BC's child protection system. Library and Archives Canada Cataloguing in Publication Data. Retrieved from http://www.mcf.gov.bc.ca/bcchildprotection/pdf/BC_Children_and_Youth_Review_Rep ort_FINAL_April_4.pdf

Ing, R. (2006). Canada's residential schools and their impacts on mothering. In lavelle-Harvard, D.M., \& Lavelle, J.C. Until our Hearts are on the Ground: Aboriginal Mothering, Oppression, Resistance and Rebirth. Toronto: Demeter Press.

Iwasaki, Y., Bartlett, J., \& O’Neil, (2004). An examination of stress among Aboriginal women and men with diabetes in Manitoba Canada. Ethnicity \& Health, 9(2), 189-212.

Jategaonkar, N. \& Ponic, P. (2011). Unsafe and unacceptable housing: health \& policy implications for women leaving violent relationships. Women's Health and Urban Life, 10(1), 32-58.

Kline, K. (1992). Child welfare law, "Best interest of the child" ideology, and First Nations. Osgoode Law Journal, 20(2), 375-425.

Krieger, J. \& Higgins, D. (2002). Housing and health: time again for public actions. American Journal of Public Health, 92(5), 758-768.

Kubik, W., Bourassa, C. \& Hampton, M. (2009). Stolen sisters, second class citizens, poor health: the legacy of colonialization in Canada. Humanity \& Society, 33, 18-34 
Landrigan, P., Rauh, V., \& Galvez, M. (2010). Environmental justice and the health of children. The Mount Sinai Journal Of Medicine, New York, 77(2), 178-187.

Laverack, G. \& Labonte, R. (2000). A planning framework for community empowerment goals within health promotion, Health Policy and Planning, 15(3) 255-262.

Lessa, L. (2002). Unraveling a Relationship: Single Motherhood and the Practices of Public Housing, Journal of Women \& Social Work, 17(3), 314-331.

Manias, E. \& Street, A. (2001). Rethinking ethnography: reconstructing nursing relationships. Methodological Issues in Nursing Research, 33(3), 232-242.

Ministry of Child and Family Development. (2009/2010). Annual Service Plan Report. Retrieved from http://www.bcbudget.gov.bc.ca/Annual_Reports/2009_2010/cfd/cfd.pdf

Ministry of Child and Family Development (n.d.). Protecting Children. Retrieved from http://www.mcf.gov.bc.ca/child_protection/

Monture, P.A. (1989). Vicious circle: child welfare and the First Nations. Canadian Journal of Women and the Law, 3, 1-17

Morse, J. M. (1993). Emerging for the data: the Cognitive process of analyses in qualitative inquiry. (p. 23-43). Thousand Oaks, California: Sage.

National Collaborating Centre for Aboriginal Health (2009-2010a). Child and Youth: Aboriginal and non-Aboriginal Children in Child Protection Services. Retrieved from http://www.nccahccnsa.ca/docs/fact\%20sheets/child\%20and\%20youth/NCCAH_fs_childhealth_EN.pdf 
National Collaborating Centre for Aboriginal Health (2009-2010b). Child Welfare Services in Canada: Aboriginal \& Mainstream. Retrieved from http://www.nccahccnsa.ca/docs/fact\%20sheets/child\%20and\%20youth/NCCAH-fs-ChildWelServCDA2EN.pdf

National Collaborating Centre for Aboriginal health (2009-2010c). Reconciliation in Aboriginal child welfare and child health. Retrieved from http://www.nccahccnsa.ca/docs/fact\%20sheets/child\%20and\%20youth/NCCAH_fs_childwelfare_EN.pdf

National Counsel of Welfare (2011). The Dollars and Sense of Solving Poverty. Volume 130. Retrieved from http://www.cnb-ncw.gc.ca/h.4m.2@-eng.jsp\#tab2a

O’Donnell, M., Nassar, N., Leonard, H., Jacoby, P., Matthews, R., Patterson, Y., \& Stanley, F. (2010). Characteristics of non-Aboriginal and Aboriginal children and families with substantiated child maltreatment: a population-based study. International Journal of Epidemiology, 39(3), 921-928.

Palmer, S. \& Cooke, W. (1996). Understanding and countering racism with First Nations children in out-of-home care. International Perspectives, LXXV(6), 709-725.

Pendakur, K., \& Pendakur, R. (2011). Aboriginal income disparity in Canada, Canadian Public Policy, 37(1), 61-83.

Pivot Legal Society (2008). Broken Promises: Parents Speak about B.C.'s Child Welfare System.

Pope, C., Ziebland, S., \& Mays, (2000). Qualitative research in health care: Analyzing qualitative data. Education and Debate, 320, 114-116.

Poudrier, J. \& Mac-Lean, R.T. (2009). 'We’ve fallen through the cracks': Aboriginal women's experiences with breast cancer through photovoice. Nursing Inquiry, 16(4), 306-317. 
Reimer-Kirkham, S.R. \& Anderson, J.M. (2002). Postcolonial nursing scholarship: from epistemology to method. Advances in Nursing Science, 25(1), 1-17.

Reimer-Kirkham, S.R., Baumbusch, J. L., Schultz, A.S.H., \& Anderson, J.M. (2007). Knowledge development and evidence-based practice: Insights and opportunities from a postcolonial feminist perspective for transformative nursing practice. Advances in Nursing Science, 30(1), 26-40.

Reutter, L., Veenstra, G., Stewart, M., Love, R,. Makwarimba, E., \& McMurray, S. (2006). Public Attributions for Poverty in Canada. Canadian Review of Sociology \& Anthropology, 43(1) 1-22.

Roberts, T. (2009). Understanding ethnography. British Journal of Midwifery, 17(5), 291-294. Royal Commission Report on Aboriginal Peoples. (1996). Report of the Royal Commission on Aboriginal Peoples: Volume 1- Looking Forward, looking back. Ottawa, ON Canada. The Commission. Retrieved from http://www.ainc-inac.gc.ca/ap/rrc-eng.as

Rothman, L. (2007). Oh Canada! Too many children in poverty for too long. Pediatrics \& Child Health, 12(8), 661- 665.

Ship, S. J., \& Norton, L. (2001). HIV/AIDS and Aboriginal women in Canada. Canadian Woman Studies, 21(2), 25-31.

Sinclair, R. (2007). Identity lost and found: lessons from the sixties scoop. First Peoples Child \& Family Review, 3(1), 65-82.

Smith, D., Varcoe, C., Edwards, N. (2005). Turning around the intergenerational impact of residential schools on Aboriginal people: Implications for health policy and practice. Canadian Journal of Nursing Research, 37(4), 38-60. 
Sorrell, J. M. \& Redmond, G. M. (1995). Interviews in qualitative nursing research: Differing approaches for ethnographic and phenomenological studies. Journal of Advanced nursing 21(6), 1117-1122.

Sullivan, R. \& Charles, G. (2010). Disproportionate representation and First Nations child welfare in Canada. The Federation of Community Social Services of BC. Research to Practice.

Smye, V. \& Browne, A.J. (2002). 'Cultural safety’ and the analysis of health policy affecting Aboriginal people. Nurse Research, 9(3), 42-56.

Thorne, S. (2008). Interpretive Description. Walnut Creek Ca: Left Coast Press.

Thorne, S. (2000). Data analysis in qualitative research. Evidence Based Learning, 3(3), 68-70.

Trocmé, N., Knoke, D., \& Blackstock, C. (2004) Pathways to the overrepresentation of Aboriginal Children in Canada's child welfare system. Social Services Review, 87(4), $577-600$.

Trocmé, N., Fallon, B., MacLaurin, B., Daciuk, J., Felstiner, C., Black, T., Tonmyr, L., Blackstock, C., Barter, C., Turcotte, D., \& Cloutier, R. (2003). Canadian Incidence Study of Reported Child Abuse and Neglect: Major Findings. Retrieved from http://www.phacaspc.gc.ca/cm-vee/csca-ecve/pdf/childabuse_final_e.pdf

Turpel, M. E. (1993). Patriarchy and paternalism: The legacy of the Canadian State for First Nations women. Canadian Journal of Women \& the Law, 6(1), 174-192.

Wharf, B. (2007). The case for a comprehensive vision for children welfare. In L.T. Foster, \& B. Wharf (Eds), People, Politics and Child Welfare in British Columbia, (pp. 226-238). Vancouver: UBC Press. 


\section{APPENDIX A: INTERVIEW FORMS FOR HEALTH CARE PROVIDERS \\ Interview Guide for Health Care Provider Participants and \\ Socio-Demographic Questions}

\section{Note to the Interviewer:}

Each interview will begin with an explanation of the consent form followed by the completion of the consent form (written signature) by the participant. Once consent is signed the interview will start with a preamble regarding the purpose of the study. The interviews will be semi-structured, with open-ended questions as well as additional ongoing follow-up questions, to insure that the same aspects are covered in all of the interviews. New questions and follow up questions will only be asked after intensive listening and after a respectful amount of time is spent waiting to insure the person is finished with speaking. The researcher will use prompts when alternative phrasing is required or if the answer provided needs more information.

\section{Interview Questions}

1. Given the purpose of this study, where would you like to start?

Prompts: Can you give me a little background about your role? Can you describe your job, as you see it?

2. What population do you work with generally? (i.e. what is your mandate?)

3. Can you give tell me about the pregnant or parenting women that you serve - what proportion of those client's are Aboriginal? Where are they from? And what are their lives generally like?

Prompt: What are some of the main reasons that clients come to seek healthcare at your centre? 
Note to the interviewer to say: As you know, the population we are studying is Aboriginal women and therefore the subsequent questions refer only to the women who identify as Aboriginal that you see.

4. Do you see many Aboriginal women accessing services at your health centre either pre or post-natally? Why or why not? Has that been changing over the years, and if so how? Prompt: What kind of services do you have for pre-natal/post-natal care?

5. One of the reasons I am doing this study is because I have been working as a neonatal nurse for the past five years as well as a clinical nursing instructor at various hospitals on pediatric units and in my experiences I believe that the threat of child apprehension is likely to deter women from accessing prenatal care and healthcare services generally. What are your thoughts or experiences in relation to that? Prompt: Can you offer any examples based on your experience working in healthcare?

6. What services are there for women when they have substance use problems, and when they 'relapse'?

Prompts: what has been your experience in relation to this?

Are there any examples you can tell me about?

7. What can mothers do when they are 'in crisis' that would keep their children safe without losing custody of their children?

Follow up: What services would be helpful for a woman in a crisis and struggling with substance use?

8. How could we better serve partners (men) dealing with issues such as anger and violence? 
9. Are there approaches that could incorporate male partners and maintain the safety of the women and children?

10. How do you think the delivery of healthcare to pregnant Aboriginal women or Aboriginal women who are having child apprehension threatened be improved?

Prompt: Do you think healthcare can be intimidating for some Aboriginal women and children?

Ideally - What kinds of approaches, strategies, or programs would be needed to better support women and would better support women in relation to accessing healthcare? 


\section{Socio-Demographic Data for Health Care Providers}

All information will be coded and anonymized. No individual identifying information will be used in any of the reports, presentations or communication.

\section{Work Experience}

1. Where are you currently working?

2. What is your current position?

3. How long have you been working at this job?

4. What is your trained profession(s)?

5. How did you get into this role?

6. What is your employment status? (full-time, part-time or casual)

7. What is the total number of year you have worked at this job placement?

8. If you work at any other agency currently, where else do you work?

9. How long have you been working in healthcare?

\section{Demographic Information:}

1. Gender:

2. Year of birth:

3. Highest level of Education:

4. City of current residence:

5. How do you identify your culture or ethnicity?

6. If self-identifying as Aboriginal, are you:

Status __ Non-Status __ Métis _ _

First Nation Affiliation if Applicable (e.g., name of Nation): 


\section{APPENDIX B: INTERVIEW FORMS FOR WOMEN \\ Interview Guide for Non-Healthcare Provider Women Participant and \\ Socio-Demographic Questions}

\section{Note to the Interviewer:}

Each interview will begin with an explanation of the consent form followed by the completion of the consent form (written signature) by the participant.. Once consent is signed the interview will start with preamble regarding the purpose of the study. The interviews will be semi-structured, with open-ended questions as well as additional ongoing follow-up questions, to insure that the same aspects are covered in all of the interviews. New questions and follow up questions will only be asked after intensive listening and after a respectful amount of time is spent waiting to insure the person is finished with speaking. The researcher will use prompts when alternative phrasing is required or if the answer provided needs more information.

Preamble: As you know, the purpose of this interview is to better understand how the concern or threat of child apprehension from protective services impacts Aboriginal women's experiences with healthcare services. We want to better understand how the threat of child apprehension affects the decisions or the interactions for Aboriginal women and families with mainstream healthcare. Basically, we are trying to learn how to better deliver healthcare to Aboriginal women and children.

\section{Questions:}

1. Can you tell me a bit about your family situation?

Prompt: Can you tell me about your children? 
2. How has it been going in terms of being "a mom" to your kids?

Prompts: How is it going in terms of seeing your kids, visiting with them?

How has it being going with the kids?

3. Tell me a bit more about your family now?

Prompt: Who lives with you?

4. What was your first pregnancy like?

Prompt: What health care did you get?

5. How was it for you to get the healthcare services you needed during your pregnancy? And after the baby was born?

6. One of the reasons I am doing this study is that I am a nurse and I have seen a fair bit of discrimination towards Aboriginal women in hospital settings, and I think that can prevent women from getting the healthcare they need. I am wondering if you have any comments about that?

Prompts: What has been your experience getting the healthcare you feel you've needed (for example hospitals, walk-in clinics)?

How did this experience affect you?

Has it affected when you choose to get healthcare services?

Where do you feel most comfortable getting healthcare?

7. I know that you have had experiences with the Ministry of Child and Family services and I am wondering if you can tell me a bit about that?

8. Has the threat of fear of having your child(ren) removed ever affected your choice to where or whether to go for healthcare services?

9. How beneficial (or not) have you found drug and alcohol counseling? 
10. What do you think would make your experience(s) with healthcare better? 


\section{Socio-Demographic Data for Women Participants}

All information will be coded and anonymized. No individual identifying information will be used in any of the reports, presentations or communication.

\section{Personal information:}

Date of birth (year only):

Place of birth:

Languages (spoken):

What would you say is your cultural background?

Are you: Status First Nations $\square \quad$ Non-status First Nations $\square \quad$ Inuit $\square \quad$ Métis $\square$ What nation are you from?

\section{Living situation}

Current living situations

House $\square$ Apartment $\square$ Rooming house $\square$ Shelter $\square$ Hotel room $\square$

Other:

Location of residence (area of city or postal code):

Do you live alone? Yes $\square$ No

If no, who do you live with?

3. Custody status with child or children

Children are living with you? Yes $\square$ No $\square$

If no, where are you living? 


\title{
APPENDIX C: CONSENT FORM FOR WOMEN
}

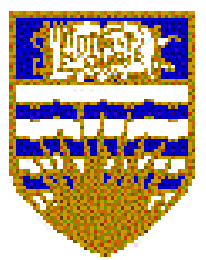

\section{THE UNIVERSITY OF BRITISH COLUMBIA}

\author{
For the Research Project \\ “Aboriginal Women's Experiences Accessing Health Care When State Apprehension of Children \\ is Being Threatened" \\ Research Team: \\ Principal Investigator Colleen Varcoe, Co-Investigators' Annette Browne, Jacqueline Denison \& \\ Laraine Michalson
}

You understand that the point of the study is to learn more about Aboriginal women's experiences using healthcare services when child apprehension is a threat. You have been identified as a possible participant through an Aboriginal Health Centre. If you are under the age of 14 we will not be able to include you in this study.

\section{What the Study Involves}

We ask to interview you in person for about 30-90 minutes. In the interview we ask you to talk about your experiences using healthcare services (e.g. health clinics, hospitals, community health nurse visits) when child apprehension is a threat. The interview will happen at a place that both you and the person doing the interview agree to and think is safe. Your participation in the study will in no way affect your guardianship status. The information will not be shared with any of your healthcare providers or staff at the health centres.

Only if you agree, the researcher will also ask if you are willing to share this interview data with the researchers on a different study. This other study is looking at healthcare delivery to Aboriginal and non-Aboriginal women and men at two Aboriginal Health centres. Once the other study is explained and only if you agree the researcher will ask you to sign the consent form for that study also.

Taking part in this study is completely voluntary. If you agree, this interview will be taperecorded. You can be interviewed without being recorded. You can ask for the recorder to be turned off at any time during the interview or have the recording erased. You may also choose to leave or stop the interview at any time. If you want, a copy of the typed-up interview with all identifying information removed can be sent to you.

In total, the researcher will interview you for somewhere between 30-90 minutes and you will receive $\$ 20.00$ to thank you for your time.

\section{Risks and Benefits}

This researcher interviewing you will be asking questions about your child or children and your experiences with healthcare and child protection services. This is likely to bring up some emotions for you. If you are very upset at any time in the interview, the interview will be stopped 
by the researcher, unless you wish otherwise. At that time, unless you wish otherwise, the researcher will accompany you to the Aboriginal Health Centre and offer to help arrange trained counseling. You will also be given a list of community services if you want to get counseling after the interview. If you tell us about any current abuse of children, we must, by law, report this to the local child protection agency. Before reporting we will discuss this with you. The benefit of being in the study is to learn how the threat of child apprehension affects Aboriginal women's decisions or experiences using healthcare services. The goal is to improve the delivery of healthcare services to Aboriginal women and children. You do not waive any of your legal rights by signing this consent form.

\section{Protecting Privacy and Confidentiality}

The information you provide to us is strictly confidential. Your confidentiality will be protected in several ways:

1. Your name will not be used in the study or in any articles written about the study. Instead of your name, a code will be used to identify each person in the study.

2. Information that could identify you will be deleted from all interview recordings, transcripts, notes, and documents.

3. Nurses, doctors and other staff in the Aboriginal Health Centre or in any other health service will not be able to see, read, or hear any of the information you provide in a way that will identify you.

4. The only people who will see the research information will be the research team members listed above. These people must keep all information in strict confidence.

5. All information will be kept securely in a locked cabinet in a research office.

6. Only if you agree the interview data will be shared with the research team involved in the Urban Aboriginal Health Care study. (See additional consent form).

7.

The results in this study will be written in a master's thesis and later in an article to be published for health care providers to read.

\section{Consent}

Your participation in the study is completely voluntary. If you decide to be in the study, and then change your mind, you are free to drop out of the study at any time with no consequences. If you have any questions or desire further information with respect to this study you may contact Colleen Varcoe (the principal investigator): Colleen.Varcoe@nursing.ubc.ca or you may contact either of the co-investigators Jacqueline Denison: Jacqueline.Denison@nursing.ubc.ca, or Annette Browne: Annette.Browne@nursing.ubc.ca.

If you have any concerns about your treatment or rights as a research subject, you are welcome to contact the Research Subject Information Line in the UBC office of Research Services at 604822-8598 or if long distance e-mail to RSIL@ ors.ubc.ca

By signing this consent form you agree to participate in the study described above. You have been given a copy of this consent form, which you can keep.

Do you give permission to the researcher to tape record the interview? Yes $\square$ No $\square$ 
Signature of Participant

Date

Please print your name 


\title{
APPENDIX D: CONSENT FORM FOR HEALTH CARE PROVIDERS
}

\author{
THE UNIVERSITY OF BRITISH COLUMBIA
}

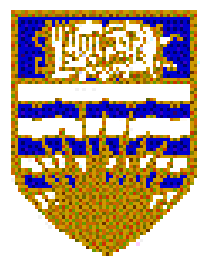

For the Research Project

"Aboriginal Women's Experiences Accessing Health Care When State Apprehension of Children is Being Threatened."

Research Team:

Principal Investigator Colleen Varcoe, Co-Investigators' Annette Browne, Jacqueline Denison \& Laraine Michalson

You understand that the purpose of the study is to learn more about Aboriginal women's experiences accessing healthcare services when child apprehension is a threat.

\section{What the Study Involves}

Interviews: The study involves taking part in an interview for approximately 30-90 minutes. The interview will take place when it is convenient for you at a mutually agreed upon place. The researcher will invite you to discuss your experiences working with Aboriginal women when their children are being investigated for apprehension. If you agree, the interview will be audiorecorded. You can request to have the interview without it being recorded. You can ask for the recorder to be turned off at any time during the interview or have any part of the recording erased.

Observations: If it is convenient for you, the researcher will "shadow" you for part of your day. Shadowing will involve accompanying you for approximately 3 or 4 hours while you provide patient care either at the health centre or during appointments at clients' homes. The observations will be of interactions or appointments with healthcare providers and Aboriginal families experiencing child apprehension investigations. These observations will not require any additional time or interfere with patient care. The patient must provide verbal consent to you in order for the researcher to remain present for observations during appointments and interactions. If the patient prefer prefers not to be observed, the researcher will immediately leave and discontinue the observations. The researcher will make short 'field notes' for recall intentions. Only if you agree to it the researcher will also ask if you are willing to share this interview data with the researchers on a larger study. This larger study is examining primary healthcare services to Aboriginal and non-Aboriginal women and men at two urban Aboriginal health centres. Once the other study is explained and if you agree the researcher will ask you to sign the consent form for the larger study as well. Your participation will only involve an interview, and if you choose to participate, a donation will be given to your organization. 


\section{Risks and Benefits}

There is no known risk for healthcare providers participating in this study. The information will not be used to evaluate your work or work performance, or the performance of your colleagues. The information will be used to shed light on how the threat of child apprehension impacts Aboriginal women accessing health care services and aims to learn strategies to better support and serve Aboriginal women and children access and quality of healthcare delivery. A copy of the results of this study will be made available. You do not waive any of your legal rights by signing this consent form.

\section{Protecting Privacy and Confidentiality}

The information you provide to us is strictly confidential. Your confidentiality will be protected in several ways:

1. Your name will not be used in the study or in any articles written about the study. Instead, a code will be given to each person in the study.

2. Information that could identify you will be deleted from all interview recordings, transcripts, notes, and documents.

3. Nurses, doctors and other staff in the health Centre will not be able to see, read, or hear any of the information you provide in a way that will identify you.

4. The only people who will see the research information will be the research team members listed above. These people are required to keep all information in strict confidence.

5. All information will be stored securely in a locked cabinet in a research office.

6. If you agree the interview data will be shared with the research team from a larger study that is looking at the delivery of primary health care services to Aboriginal and non-Aboriginal men and women at two urban Aboriginal health centres (see additional consent form)

The results of this study will be written in a master's thesis and later in an article to be published for health care providers to read.

\section{Consent}

Your participation in the study is completely voluntary. Your decision to participate will in no way influence your employment or performance evaluation.

If you decide to be in the study, and then change your mind, you are free to drop out of the study at any time with no consequences.

If you have any questions or desire further information with respect to this study you may contact Colleen Varcoe (the principal investigator): Colleen.Varcoe@ nursing.ubc.ca or you may contact either of the co-investigators Jacqueline Denison: Jacqueline.Denison@ @ursing.ubc.ca, or Annette Browne: Annette.Browne@nursing.ubc.ca.

If you had any concerns about your treatment or rights as a research subject, you are welcome to contact the Research Subject Information Line in the UBC office of Research Services at 604-822-8598 or if long distance e-mail to RSIL@ors.ubc.ca

By signing this consent form you agree to participate in the study described above. You have received a copy of this consent form, which you can keep. 
Do you give permission to the researcher to audio- record the interview? Yes $\square$ No $\square$

Signature of Participant

Date

Please print your name 